\title{
CATÉGORIES DÉRIVÉES ET VARIÉTÉS DE DELIGNE-LUSZTIG
}

\author{
CÉDRIC BONNAFÉ ET RAPHAËL ROUQUIER
}

TABLE DES MATIÈRES

1. Introduction

2. Notations générales

2.1. Groupes, monoïdes

2.2. Anneaux, corps

2.3. Catégories dérivées

2.4. Algèbres

2.5. Groupes finis

2.6. Groupes algébriques

2.7. Faisceaux

3. Variétés et groupes finis

3.1. Définitions

3.2. Foncteurs

3.3. Composition

4. Préliminaires sur les groupes réductifs finis

4.1. Groupes réductifs

4.2. Racines, groupe de Weyl, groupe de tresses 9

4.3. Suites d'éléments de $W \quad 10$

4.4. Tores et caractères 10

5. Variétés de Deligne-Lusztig généralisées 18

5.1. Comparaison de modèles 18

5.2. Définition 20

5.3. Liens entre les groupes $\mathbf{G}, \tilde{\mathbf{G}}$ et $\hat{\mathbf{G}} \quad 22$

6. Recollements 24

6.1. Construction 24

6.2. Le cas général 25

6.3. Recollement de recollements 26

6.4. Remarques 27

7. Calculs de monodromie 28

8. Induction de Deligne-Lusztig et séries rationnelles 31

8.1. Orthogonalité 31

8.2. Profondeur 33

Date: Décembre 2001. 
9. Engendrement de $\Lambda \mathbf{G}^{F}$-parf 35

9.1. Engendrement de catégories de complexes parfaits. 35

9.2. Décomposition en séries 36

10. Foncteurs de Lusztig 38

10.1. Définition 38

10.2. Transitivité et séries rationnelles 39

$\begin{array}{ll}\text { 10.3. Equivalence de Morita } & 40\end{array}$

11. Changement de point de vue 43

11.1. Notations 43

11.2. Rapport avec les groupes $\mathbf{L}_{I}^{\dot{v} F} \quad 44$

11.3. Séries rationnelles 45

11.4. Equivalence de Morita $\quad 46$

11.5. Décomposition de Jordan 47

12. Appendice : quelques lemmes géométriques 48

12.1. Autour de la formule de Künneth 48

12.2. Quasi-affinité $\quad 49$

12.3. Diviseurs à croisements normaux 50

$\begin{array}{ll}\text { Références } & 51\end{array}$

\section{INTRODUCTION}

Les classes de conjugaison d'un groupe $G$ réductif connexe sur un corps fini se décrivent à partir d'une classe d'éléments semi-simples et une classe d'éléments unipotents du centralisateur d'un élément semi-simple (décomposition de Jordan). Deligne et Lusztig ont construit une décomposition analogue des caractères irréductibles de $G$ : ils sont paramétrés par une classe semi-simple du groupe dual et un caractère unipotent du centralisateur d'un élément de cette classe. Nous démontrons ici un résultat du même type pour des représentations modulaires de $G$.

Choisissons un nombre premier $\ell$ différent de la caractéristique du corps de définition du groupe. Broué et Michel ont montré que la décomposition en $\ell$-blocs des caractères raffine la décomposition suivant la $\ell^{\prime}$-partie de la classe semi-simple associée à un caractère. Broué [Br] a conjecturé que les foncteurs d'induction de Deligne-Lusztig devaient produire une équivalence de Morita entre la réunion des blocs associés à un $\ell^{\prime}$-élément semi-simple $s$ du groupe dual et la réunion des blocs unipotents du groupe dual du centralisateur de $s$ (lorsque ce dernier est un sous-groupe de Levi).

La motivation de ce travail est la preuve de cette conjecture sur la décomposition de Jordan des blocs. Nous démontrons qu'un bloc d'un groupe réductif connexe sur un corps fini (en caractéristique transverse) est Morita-équivalent à un bloc quasi-isolé d'un sousgroupe de Levi.

Comme l'avait montré Broué, le problème est géométrique. On dispose de foncteurs d'induction de Deligne-Lusztig, qui envoient représentations sur complexes de représentations. 
Le problème est de démontrer que, sous certaines hypothèses, les complexes qui interviennent sont concentrés en un degré. Broué a ramené le problème à établir la concentration en degré moitié de la cohomologie de certains faisceaux localement constants sur des variétés de Deligne-Lusztig. Ce problème a été résolu, dans le cas de faisceaux modérément ramifiés associés à des caractères de tores, par Deligne et Lusztig [DeLu] et notre travail consiste à résoudre ce problème en général (théorème 10.7).

Le problème qui se pose est double. D'abord, les variétés concernées $X$ sont associées à des variétés de drapeaux paraboliques et on manque d'une compactification lisse avec diviseurs à croisements normaux. Ensuite, les systèmes locaux $\mathcal{F}$ en jeu sont sauvagement ramifiés. Du coup, il ne suffit plus de montrer que le faisceau ne s'étend pas à l'infini dans une bonne compactification pour déduire que sa cohomologie est concentrée en degré moitié.

Nous parvenons à contourner ces difficultés en deux étapes.

Tout d'abord, nous introduisons des variétés $f_{i}: X_{i} \rightarrow X$ au-dessus de $X$ et des systèmes locaux modérément ramifiés $\mathcal{F}_{i}$ sur $X_{i}$ tels que $\mathcal{F}$ est dans la sous-catégorie triangulée de $D^{b}(X)$ engendrée par les $\mathrm{R}\left(f_{i}\right)_{*} \mathcal{F}_{i}$. Nous déduisons cela d'un résultat présentant un intérêt indépendant : la catégorie des complexes parfaits de modules pour $G$ est engendrée par les images des foncteurs de Deligne-Lusztig (§9).

Ensuite, une étude précise de la ramification à l'infini des faisceaux $\mathcal{F}_{i}$ nous permet de conclure. Plus précisément, on dispose d'une immersion ouverte $j_{i}: X_{i} \rightarrow Y_{i}$ et d'un morphisme propre $Y_{i} \rightarrow \bar{X}$, où $j: X \rightarrow \bar{X}$ est une compactification de $X$ (singulière en général). L'étude de la ramification montre que $\left.\left(\mathrm{R}\left(j_{i}\right)_{*} \mathcal{F}_{i}\right)_{\mid f_{i}^{-1}(\bar{X}-X)}\right)=0$, ce qui suffit pour établir que le morphisme canonique $j_{!} \mathcal{F} \rightarrow \mathrm{R} j_{*} \mathcal{F}$ est un quasi-isomorphisme.

Pour comprendre précisément comment les systèmes locaux $\mathcal{F}_{i}$ associés à des caractères de tores se ramifient à l'infini $(\S 7)$, nous construisons de nouvelles variétés de type DeligneLusztig (§6). Le recollement de revêtements repose sur la construction d'isomorphismes canoniques entre quotients de tores. C'est l'objet de la partie $\S 4.4$, où nous obtenons aussi une nouvelle approche de la conjugaison rationnelle de caractères de tores.

Les résultats principaux sont contenus dans les parties $\S 10$ et $\S 11$. Dans les parties $\S 7$ et $\S 8.2$, nous supposons que l'anneau de coefficients $\Lambda$ est un corps. L'appendice $\S 12$ regroupe quelques résultats géométriques.

Le lecteur verra sans peine l'importance considérable de notre dette à l'égard de l'article fondateur de Deligne et Lusztig [DeLu]. Nous utilisons aussi certaines techniques développées par Digne et Michel [DiMiRo] (variétés associées à des suites d'éléments du groupe de Weyl et compactifications partielles).

Dans un second travail, nous aborderons les applications à la théorie locale des blocs (morphisme de Brauer, équivalences splendides, finitude des algèbres de source, engendrement de la catégorie dérivée, variation du sous-groupe parabolique, groupes non connexes).

Nous remercions Marc Cabanes et Michel Enguehard pour leurs nombreux commentaires et suggestions. 


\section{Notations GÉNÉRALES}

2.1. Groupes, monoïdes. Soit $G$ un groupe. Nous noterons $G^{\text {op }}$ le groupe opposé à $G$. Pour $M$ un $G$-ensemble, on note $M^{\text {op }}$ le $G^{\text {op }}$-ensemble, restriction de $M$ à travers l'isomorphisme $G^{\text {op }} \stackrel{\sim}{\rightarrow} G, g \mapsto g^{-1}$.

Pour $g \in G$, nous noterons $(g)$ (ou $(g)_{G}$ s'il y a confusion possible) la classe de conjugaison de $g$ dans $G$. Si $g$ et $g^{\prime}$ sont deux éléments de $G$ nous écrirons $g \sim g^{\prime}$ (ou $g \sim_{G} g^{\prime}$ ) pour dire que $g$ et $g^{\prime}$ sont conjugués dans $G$.

Si $X$ est une partie de $G$ stable par conjugaison, $X / \sim\left(\right.$ ou $X / \sim_{G}$ ) désignera l'ensemble des classes de conjugaison de $G$ contenues dans $X$. Si $\pi$ est un ensemble de nombres premiers (ou un nombre premier) nous noterons $X_{\pi}$ (respectivement $X_{\pi^{\prime}}$ ) l'ensemble des $\pi$-éléments (respectivement $\pi^{\prime}$-éléments) de $X$. Si $g \in G$ est d'ordre fini, nous noterons $g_{\pi}$ et $g_{\pi^{\prime}}$ les uniques éléments de $G_{\pi}$ et $G_{\pi^{\prime}}$ respectivement tels que $g=g_{\pi} g_{\pi^{\prime}}=g_{\pi^{\prime}} g_{\pi}$.

Si $E$ est un ensemble, nous noterons $\Sigma(E)$ le monoïde libre sur l'ensemble $E$, c'est-à-dire l'ensemble des suites finies d'éléments de $E$ muni de la loi de concaténation. Son élément neutre sera noté (). Si $\sigma$ est un endomorphisme de $E$, nous noterons parfois ${ }^{\sigma} e$ l'élément $\sigma(e)$.

2.2. Anneaux, corps. Dans cet article, nous fixons une fois pour toutes deux nombres premiers distincts $p$ et $\ell$. Nous noterons $\mathbf{F}$ une clôture algébrique du corps fini à $p$ éléments $\mathbf{F}_{p}$. Nous fixons une puissance $q$ de $p$ et notons $\mathbf{F}_{q}$ le sous-corps de $\mathbf{F}$ à $q$ éléments. Nous appellerons variété (respectivement groupe algébrique) une variété algébrique quasiprojective définie (respectivement un groupe algébrique défini) sur le corps $\mathbf{F}$.

Nous fixons aussi une extension algébrique $K$ du corps $\ell$-adique $\mathbf{Q}_{\ell}$. Soit $R$ son anneau d'entiers sur $\mathbf{Z}_{\ell}$ et soit $k$ le corps résiduel de $R$ : c'est une extension algébrique du corps fini à $\ell$ éléments $\mathbf{F}_{\ell}$. Nous notons $\mathfrak{l}$ l'idéal maximal de $R$. Nous supposerons que le corps $K$ est "assez gros", c'est-à-dire que, pour tout groupe fini $H$ rencontré dans cet article, $K$ contient les racines $e$-ièmes de l'unité, où $e$ est l'exposant de $H$.

Nous fixons une fois pour toutes un isomorphisme

$$
\imath:(\mathbf{Q} / \mathbf{Z})_{p^{\prime}} \stackrel{\sim}{\longrightarrow} \mathbf{F}^{\times}
$$

ainsi qu'un morphisme injectif

$$
\jmath:(\mathbf{Q} / \mathbf{Z})_{p^{\prime}} \hookrightarrow \overline{\mathbf{Q}}_{\ell}^{\times},
$$

où $\overline{\mathbf{Q}}_{\ell}$ est une clôture algébrique de $K$. Soit

$$
\kappa=\jmath \circ \imath^{-1}: \mathbf{F}^{\times} \hookrightarrow \overline{\mathbf{Q}}_{\ell}^{\times} .
$$

Dans tout cet article, $\Lambda$ sera l'un des anneaux $K$ ou $R / \mathfrak{l}^{n}(n \geq 0)$.

2.3. Catégories dérivées. Si $\mathcal{A}$ est une catégorie additive, nous noterons $\mathrm{K}(\mathcal{A})$ son groupe de Grothendieck et $K^{b}(\mathcal{A})$ la catégorie homotopique des complexes bornés d'objets de $\mathcal{A}$.

Si $\mathcal{C}$ est une catégorie abélienne, nous noterons $\mathrm{K}(\mathcal{C})$ son groupe de Grothendieck, $D^{b}(\mathcal{C})$ (respectivement $D^{-}(\mathcal{C})$ ) la catégorie dérivée des complexes à cohomologie bornée (respectivement bornée supérieurement) et $\mathcal{C}$-parf la catégorie de ses complexes parfaits; 
$\mathrm{K}(\mathcal{C})$ est aussi le groupe de Grothendieck de la catégorie triangulée $D^{b}(\mathcal{C})$. Si $E$ est un ensemble d'objets d'une catégorie triangulée $D$, nous appellerons sous-catégorie de $D$ engendrée par $E$ la plus petite sous-catégorie triangulée pleine de $D$ stable par facteurs directs et contenant $E$. Nous identifierons $\mathcal{C}$ avec la sous-catégorie pleine de $D^{b}(\mathcal{C})$ des complexes concentrés en degré 0 via le foncteur canonique.

Lorsque $\mathcal{C}$ a assez de projectifs, étant donnés $C$ et $C^{\prime}$ deux objets de $D^{b}(\mathcal{C})$, nous noterons $\mathrm{RHom}^{\bullet}\left(C, C^{\prime}\right)$ le complexe total associé au complexe double des homomorphismes d'une résolution projective de $C$ vers $C^{\prime}$.

2.4. Algèbres. Soient $A$ et $B$ deux $\Lambda$-algèbres. Nous noterons $A^{\text {op }}$ l'algèbre opposée à $A$. Par un $A$-module, nous entendrons un module à gauche pour $A$. Tous les modules considérés seront de type fini. Nous noterons $A$-mod la catégorie des $A$-modules. On identifie la catégorie des $(A, B)$-bimodules à celle des $\left(A \otimes_{\Lambda} B^{\mathrm{op}}\right)$-modules. Nous noterons $\mathrm{K}(A), D^{b}(A), D^{-}(A)$ et $A$-parf les constructions obtenues pour $\mathcal{C}=A$-mod.

Pour $M$ un complexe borné de $A$-modules, nous définissons le complexe de $A$-modules à droite $M^{*}=\mathrm{R} \mathrm{Hom}_{\Lambda}^{\bullet}(M, \Lambda)$.

Nous noterons $A$-proj la sous-catégorie additive pleine de $A$-mod formée par les $A$ modules projectifs.

2.5. Groupes finis. Si $G$ est un groupe fini, nous désignerons par $\operatorname{Irr}_{K} G$ l'ensemble de ses caractères irréductibles sur $K$. Nous identifierons alors $\mathrm{K}(K G)$ avec $\mathbf{Z} \operatorname{Irr}_{K} G$, le Zmodule libre de base $\operatorname{Irr}_{K} G$. Le produit scalaire sur $\mathrm{K}(K G)$ faisant de $\operatorname{Irr}_{K} G$ une base orthonormale sera noté $\langle\cdot, \cdot\rangle_{G}$. Si $\theta: G \rightarrow \Lambda^{\times}$est un caractère linéaire de $G$, nous noterons $\Lambda_{\theta}$ le $\Lambda G$-module sur lequel un élément $g \in G$ agit par multiplication par $\theta(g)$, et $e_{\theta}$ (ou $e_{\Lambda, \theta}$, ou $e_{\Lambda, \theta}^{G}$ s'il est nécessaire de préciser) l'idempotent primitif central de $\Lambda G$ qui agit comme l'identité sur $\Lambda_{\theta}$.

2.6. Groupes algébriques. Soit $\mathbf{H}$ un groupe algébrique. On désignera par $\mathbf{H}^{\circ}$ sa composante neutre et $\mathbf{H}_{\text {sem }}$ l'ensemble de ses éléments semi-simples. Si $h \in \mathbf{H}$, nous noterons $C_{\mathbf{H}}^{\circ}(h)$ la composante neutre de son centralisateur dans $\mathbf{H}$. Si $\mathbf{H}$ est abélien et si $F$ est un endomorphisme de $\mathbf{H}$, alors nous notons $N_{F^{n} / F}: \mathbf{H} \rightarrow \mathbf{H}, h \mapsto h^{F} h \ldots{ }^{F^{n-1}} h$ le morphisme norme de $F^{n} \grave{a} F$ (où $n \in \mathbf{N}^{*}$ ).

2.7. Faisceaux. Soit $\mathbf{X}$ une variété et soit $A$ une $\Lambda$-algèbre. Nous noterons $D_{A}^{?}(\mathbf{X})$ la sous-catégorie pleine de la catégorie dérivée de la catégorie des faisceaux de $A$-modules sur $X$ formée des complexes à cohomologie constructible, bornée si $?=b$, bornée supérieurement si $?=-$.

Notons $\pi_{\mathbf{X}}: \mathbf{X} \rightarrow$ Spec $\mathbf{F}$ le morphisme canonique. Un $A$-module $M$ sera identifié avec le $A$-faisceau constant sur $\operatorname{Spec} \mathbf{F}$ associé à $M$. Nous posons alors $M_{\mathbf{X}}=\pi_{\mathbf{X}}^{*} M$ : c'est le $A$-faisceau constant sur $\mathbf{X}$ associé au $A$-module $M$. De même, un complexe de $A$-modules $C$ sera identifié avec le complexe de faisceaux constants sur $\operatorname{Spec} \mathbf{F}$ correspondant et nous noterons $C_{\mathbf{X}}=\pi_{\mathbf{X}}^{*} C$. 
Nous noterons $\mathrm{R} \Gamma(\mathbf{X}, C)=\mathrm{R}\left(\pi_{\mathbf{X}}\right)_{*}(C)$ (respectivement $\mathrm{R} \Gamma_{c}(\mathbf{X}, C)=\mathrm{R}\left(\pi_{\mathbf{X}}\right)_{!}(C)$ ) le complexe de cohomologie (respectivement de cohomologie à support compact) d'un complexe $C \in D_{A}^{b}(\mathbf{X})$. Nous écrirons $\mathrm{R} \Gamma(\mathbf{X})$ (respectivement $\mathrm{R} \Gamma_{c}(\mathbf{X})$ ) pour $\mathrm{R} \Gamma\left(\mathbf{X}, \Lambda_{\mathbf{X}}\right)$ (respectivement $\left.\mathrm{R} \Gamma_{c}\left(\mathbf{X}, \Lambda_{\mathbf{X}}\right)\right)$. Pour $G$ un groupe fini agissant sur $\mathbf{X}$ de telle sorte que les stabilisateurs des points de $\mathbf{X}$ sont des sous-groupes de $G$ d'ordre inversible dans $\Lambda$, alors $\mathrm{R} \Gamma_{c}(\mathbf{X})$ est un complexe parfait de $\Lambda G$-modules [DeLu, §3.8].

\section{VARIÉTÉS ET GROUPES FINIS}

Les variétés de Deligne-Lusztig sont munies d'actions à gauche et à droite de groupes réductifs finis. Leur cohomologie permet alors de définir des foncteurs entre catégories de représentations. Nous rappelons ici dans un cadre général comment définir ces foncteurs ainsi que quelques-unes de leurs propriétés élémentaires.

3.1. Définitions. Nous fixons trois groupes finis $G, H$ et $K$. Une $G$-variété (respectivement une variété- $G$ ) est une variété sur laquelle $G$ agit à gauche (respectivement à droite). Une $G$-variété- $H$ est à la fois une $G$-variété et une variété- $H$, les actions de $G$ et $H$ com-

mutant. Si $\mathbf{X}$ est une variété- $G$ et si $\mathbf{Y}$ est une $G$-variété, nous noterons $\mathbf{X} \times_{G} \mathbf{Y}$ la variété quotient de $\mathbf{X} \times \mathbf{Y}$ par $G$, le groupe $G$ agissant à gauche sur $\mathbf{X} \times \mathbf{Y}$ par

$$
\begin{array}{ccc}
G \times(\mathbf{X} \times \mathbf{Y}) & \longrightarrow & \mathbf{X} \times \mathbf{Y} \\
(g,(x, y)) & \longmapsto & \left(x g^{-1}, g y\right) .
\end{array}
$$

Une $G$-variété, ou une variété- $G$, est dite régulière si le groupe $G$ agit librement.

3.2. Foncteurs. Soit $\mathbf{X}$ une $G$-variété- $H$. Les complexes $\mathrm{R} \Gamma_{c}(\mathbf{X})$ et $\mathrm{R} \Gamma(\mathbf{X})$ sont des complexes de $(\Lambda G, \Lambda H)$-bimodules. Ils induisent donc deux foncteurs

et

$$
\begin{array}{cccc}
\mathcal{R}_{H}^{G}(\mathbf{X}): & D^{-}(\Lambda H) & \longrightarrow & D^{-}(\Lambda G) \\
C & \longmapsto & \mathrm{R}_{c}(\mathbf{X}) \otimes_{\Lambda H}^{\mathrm{L}} C \\
& & \\
\mathcal{S}_{H}^{G}(\mathbf{X}): & D^{-}(\Lambda H) & \longrightarrow & D^{-}(\Lambda G) \\
C & \longmapsto & \mathrm{R} \Gamma(\mathbf{X}) \otimes_{\Lambda H}^{\mathrm{L}} C .
\end{array}
$$

On considérera aussi le foncteur

$$
\begin{aligned}
& \mathcal{F}_{H}^{\mathbf{X}}: D^{-}(\Lambda H) \quad \longrightarrow \quad D_{\Lambda}^{-}(\mathbf{X} / H) \\
& M \quad \longmapsto \pi_{*} \Lambda_{\mathbf{X}} \otimes_{\Lambda H}^{\mathrm{L}} M_{\mathbf{X} / H} \text {. }
\end{aligned}
$$

où $\pi: \mathbf{X} \rightarrow \mathbf{X} / H$ est le morphisme quotient.

Remarque 3.1. Supposons que $\mathbf{X}$ est une variété- $H$ régulière. Alors, le foncteur $\mathcal{F}_{H}^{\mathbf{X}}$ envoie un $\Lambda H$-module sur un $\Lambda$-système local sur la variété $\mathbf{X} / H$. En outre, les foncteurs $\mathcal{R}_{H}^{G}(\mathbf{X}), \mathcal{S}_{H}^{G}(\mathbf{X})$ et $\mathcal{F}_{H}^{\mathbf{X}}$ se restreignent en des foncteurs entre catégories dérivées bornées. 
Lemme 3.2. On a des isomorphismes canoniques de foncteurs de $D^{b}(\Lambda H)$ vers $D^{-}(\Lambda G)$,

$$
\begin{aligned}
& \mathrm{R}\left(\pi_{\mathbf{X} / H}\right)_{!} \circ \mathcal{F}_{H}^{\mathbf{X}} \simeq \mathcal{R}_{H}^{G}(\mathbf{X}) \\
& \mathrm{R}\left(\pi_{\mathbf{X} / H}\right)_{*} \circ \mathcal{F}_{H}^{\mathbf{X}} \simeq \mathcal{S}_{H}^{G}(\mathbf{X}) .
\end{aligned}
$$

Si les stabilisateurs de points de $\mathbf{X}$ sous l'action de $H$ sont d'ordre inversible dans $\Lambda$, alors, pour $M$ un $\Lambda H$-module, on a un isomorphisme canonique

$$
\mathcal{F}_{H}^{\mathbf{X}} M \simeq \pi_{*} \Lambda_{\mathbf{X}} \otimes_{\Lambda H} M_{\mathbf{X} / H}
$$

Démonstration. Puisque $\mathrm{R}\left(\pi_{\mathbf{X} / H}\right) ! \circ \pi_{*} \simeq \mathrm{R}\left(\pi_{\mathbf{X} / H}\right)$ ! $\circ \mathrm{R} \pi_{!} \simeq \mathrm{R}\left(\pi_{\mathbf{X}}\right)$ ! , (noter que $\pi_{*}=\pi_{\text {! }}$ est un foncteur exact car $\pi$ est un morphisme fini), la commutativité du premier diagramme est équivalente à la propriété suivante :

$$
\mathrm{R}\left(\pi_{\mathbf{X} / H}\right) !\left(\pi_{*} \Lambda_{\mathbf{X}} \otimes_{\Lambda H}^{\mathrm{L}} \pi_{\mathbf{X} / H^{*}}^{*}\right) \simeq\left(\mathrm{R}\left(\pi_{\mathbf{X} / H}\right) ! \pi_{*} \Lambda_{\mathbf{X}}\right) \otimes_{\Lambda H}^{\mathrm{L}}-
$$

Cela résulte du lemme 12.1 .

La commutativité du deuxième diagramme se démontre de la même manière mais en utilisant cette fois le lemme 12.2 au lieu du lemme 12.1.

Pour la dernière partie du lemme, on utilise que les fibres de $\pi_{*} \Lambda_{\mathbf{X}}$ sont des $\Lambda H$-modules projectifs.

3.3. Composition. Soit maintenant $\mathbf{Y}$ une $H$-variété- $K$. La variété $\mathbf{X} \times_{H} \mathbf{Y}$ est une $G$-variété- $K$ et la formule de Künneth montre que

$$
\mathrm{R} \Gamma_{c}(\mathbf{X}) \otimes_{\Lambda}^{\mathrm{L}} \mathrm{R} \Gamma_{c}(\mathbf{Y}) \simeq \mathrm{R} \Gamma_{c}(\mathbf{X} \times \mathbf{Y})
$$

Supposons jusqu'à la fin du $§ 3.3$ que les stabilisateurs des points de $\mathbf{X} \times \mathbf{Y}$ sous l'action diagonale de $H$ sont d'ordre inversible dans $\Lambda$. Alors, $\mathrm{R} \Gamma_{c}(\mathbf{X} \times \mathbf{Y}) \otimes_{\Lambda H}^{\mathrm{L}} \Lambda \simeq \mathrm{R} \Gamma_{c}\left(\mathbf{X} \times_{H} \mathbf{Y}\right)$ (cf lemme 3.2). On en déduit, après application du foncteur $-\otimes_{\Lambda H}^{\mathrm{L}} \Lambda$ à la formule de Künneth, que

$$
\mathrm{R} \Gamma_{c}(\mathbf{X}) \otimes_{\Lambda H}^{\mathrm{L}} \mathrm{R} \Gamma_{c}(\mathbf{Y}) \simeq \mathrm{R} \Gamma_{c}\left(\mathbf{X} \times_{H} \mathbf{Y}\right)
$$

dans $D^{b}(\Lambda G \otimes \Lambda K)$. En particulier, on obtient

$$
\mathcal{R}_{H}^{G}(\mathbf{X}) \circ \mathcal{R}_{K}^{H}(\mathbf{Y}) \simeq \mathcal{R}_{K}^{G}\left(\mathbf{X} \times_{H} \mathbf{Y}\right)
$$

\section{Préliminaires SuR LeS Groupes RÉDUCtifs Finis}


4.1. Groupes réductifs. Dorénavant, et ce jusqu'à la fin de cet article, nous fixons un groupe réductif connexe $\mathbf{G}$ muni d'une isogénie $F: \mathbf{G} \rightarrow \mathbf{G}$ dont une puissance $F^{\delta}$ est l'endomorphisme de Frobenius sur $\mathbf{G}$ associé à une structure rationnelle sur $\mathbf{F}_{q}$. Il est à noter que les entiers $q$ et $\delta$ ne sont pas uniquement déterminés par la donnée de $\mathbf{G}$ et $F$. Cependant, le réel positif $q^{1 / \delta}$ l'est. Soit $\mathbf{B}$ un sous-groupe de Borel $F$-stable de $\mathbf{G}$ et soit $\mathbf{T}$ un tore maximal $F$-stable de $\mathbf{B}$. Le radical unipotent de $\mathbf{B}$ sera noté $\mathbf{U}$. On note $\left(\mathbf{G}^{*}, \mathbf{T}^{*}, F^{*}\right)$ un triplet dual de $(\mathbf{G}, \mathbf{T}, F)$ [DeLu, définition 5.21].

Le sujet général de cet article est l'étude des représentations modulaires du groupe fini $\mathbf{G}^{F}$. Pour s'y préparer, nous fixons dans ce $\S 4$ quelques notations (racines, groupe de Weyl...), avant d'établir quelques résultats combinatoires sur les tores $F$-stables de G (cf §4.4). Nous introduisons tout d'abord deux groupes réductifs connexes, du même type que $\mathbf{G}$, qui joueront le rôle d'auxiliaires techniques. Ils seront utiles dans certaines constructions (séries rationnelles, recollement de variétés de Deligne-Lusztig...) et leur usage facilitera certaines démonstrations.

Remarque 4.1. Si $\mathbf{H}$ est un sous-groupe fermé connexe distingué de $\mathbf{G}$ (mais non nécessairement $F$-stable), et si on pose $\mathbf{K}=\left\{g \in \mathbf{G} \mid g^{-1} F(g) \in \mathbf{H}\right\}$, alors il est immédiat que $\mathbf{K}$ est un sous-groupe fermé de $\mathbf{G}$ contenant $\mathbf{G}^{F}$. De plus,

$$
\mathbf{K}=\mathbf{G}^{F} \cdot \mathbf{K}^{\circ} \text {. }
$$

En effet, l'application de Lang $\mathbf{G} \rightarrow \mathbf{G}, g \mapsto g^{-1} F(g)$ est un morphisme fini surjectif, donc l'image de $\mathbf{K}^{\circ}$ par cette application est une sous-variété irréductible fermée de dimension $\operatorname{dim} \mathbf{K}^{\circ}=\operatorname{dim} \mathbf{K}=\operatorname{dim} \mathbf{H}$ de $\mathbf{H}$. Or, $\mathbf{H}$ est ici supposé connexe, donc l'image de $\mathbf{K}^{\circ}$ est $\mathbf{H}$. Le résultat découle alors du fait que les fibres de l'application de Lang sont des $\mathbf{G}^{F}$-orbites (pour l'action de $\mathbf{G}^{F}$ par multiplication à gauche).

4.1.1. On sait [DeLu, preuve du corollaire 5.18] qu'il existe un groupe réductif connexe $\tilde{\mathbf{G}}$ muni d'une isogénie $F: \tilde{\mathbf{G}} \rightarrow \tilde{\mathbf{G}}$ telle que $F^{\delta}$ soit l'endomorphisme de Frobenius sur $\tilde{\mathbf{G}}$ associé à une $\mathbf{F}_{q}$-structure et vérifiant les conditions suivantes :

(1) $\mathbf{G}$ est un sous-groupe fermé F-stable de $\tilde{\mathbf{G}}$, contenant le groupe dérivé de $\tilde{\mathbf{G}}$;

(2) le centre $Z(\tilde{\mathbf{G}})$ de $\tilde{\mathbf{G}}$ est connexe.

On a alors $\tilde{\mathbf{G}}=\mathbf{G} \cdot Z(\tilde{\mathbf{G}})$. Nous noterons $\tilde{\mathbf{T}}$ (respectivement $\tilde{\mathbf{B}}$ ) l'unique tore maximal (respectivement sous-groupe de Borel) de $\tilde{\mathbf{G}}$ contenant $\mathbf{T}$ (respectivement B). D'autre part, nous noterons $\left(\tilde{\mathbf{G}}^{*}, \tilde{\mathbf{T}}^{*}, F^{*}\right)$ un triplet dual de $(\tilde{\mathbf{G}}, \tilde{\mathbf{T}}, F)$. L'inclusion $\mathbf{G} \hookrightarrow \tilde{\mathbf{G}}$ induit un morphisme surjectif de groupes algébriques $i^{*}: \tilde{\mathbf{G}}^{*} \rightarrow \mathrm{G}^{*}$ commutant avec $F$, dont le noyau est un tore central de $\tilde{\mathbf{G}}^{*}$ et tel que $i^{*}\left(\tilde{\mathbf{T}}^{*}\right)=\mathbf{T}^{*}$. Ce morphisme $i^{*}$ est bien défini à conjugaison près par un élément de $\mathbf{T}^{* F^{*}}$. 
4.1.2. De manière duale, il existe un groupe réductif connexe $\hat{\mathbf{G}}$ muni d'une isogénie $F: \hat{\mathbf{G}} \rightarrow \hat{\mathbf{G}}$ telle que $F^{\delta}$ soit l'endomorphisme de Frobenius sur $\hat{\mathbf{G}}$ associé à une $\mathbf{F}_{q^{-}}$ structure et un tore central $F$-stable $\mathbf{C}$ de $\hat{\mathbf{G}}$ vérifiant les deux propriétés suivantes :

(1) $\mathbf{G}=\hat{\mathbf{G}} / \mathbf{C}$;

(2) Toute coracine $\alpha^{\vee}: \mathbf{F}^{\times} \rightarrow \hat{\mathbf{G}}$ relative à un tore maximal de $\hat{\mathbf{G}}$ est injective.

Notons que si le groupe dérivé de $\mathbf{G}$ est simplement connexe, alors toute coracine est injective.

Nous noterons $\rho: \hat{\mathbf{G}} \rightarrow \mathbf{G}$ la projection canonique. Nous noterons $\hat{\mathbf{T}}$ (respectivement $\hat{\mathbf{B}}$ ) le tore maximal (respectivement le sous-groupe de Borel) de $\hat{\mathbf{G}}$ égal à $\rho^{-1}(\mathbf{T})$ (respectivement $\left.\rho^{-1}(\mathbf{B})\right)$.

Plus généralement, nous noterons ? (respectivement ?) l'objet associé à $\tilde{G}$ (respectivement $\hat{\mathbf{G}}$ ) défini de la même façon que l'objet ? associé à $\mathbf{G}$.

4.2. Racines, groupe de Weyl, groupe de tresses. Soit $W$ le groupe de Weyl de G relatif à $\mathbf{T}$, soit $n \mapsto \bar{n}$ le morphisme canonique $N_{\mathbf{G}}(\mathbf{T}) \rightarrow W$, soit $\Phi$ le système de racines de $\mathbf{G}$ relatif à $\mathbf{T}$ et soit $\Phi^{\vee}$ le système de coracines. Soit $\Phi^{+}$(respectivement $\Delta$ ) le système de racines positives (respectivement la base) de $\Phi$ associé (respectivement associée) à $\mathbf{B}$. Si $\alpha \in \Phi$, nous noterons $\alpha^{\vee}$ sa coracine, $\mathbf{T}_{\alpha^{\vee}}$ le sous-tore de $\mathbf{T}$ image de $\alpha^{\vee}$, $\mathbf{U}_{\alpha}$ le sous-groupe unipotent à un paramètre normalisé par $\mathbf{T}$ associé à $\alpha, s_{\alpha}$ la réflexion de $W$ par rapport à $\alpha$ et $\mathbf{G}_{\alpha}$ le sous-groupe de $\mathbf{G}$ engendré par $\mathbf{U}_{\alpha}$ et $\mathbf{U}_{-\alpha}$. Nous noterons $\phi: \Phi \rightarrow \Phi$ la bijection définie par ${ }^{F} \mathbf{U}_{\alpha}=\mathbf{U}_{\phi(\alpha)}$ pour tout $\alpha \in \Phi$. Nous noterons $Y(\mathbf{T})$ le réseau des sous-groupes à un paramètre de $\mathbf{T}$.

Soit $S$ l'ensemble des réflexions simples de $W$ et soit $\bar{S}=S \cup\{1\}$. Si $w \in W$, nous noterons $l(w)$ la longueur de $w$ relativement à $S$ (c'est aussi le cardinal de l'ensemble $\left.\left\{\alpha \in \Phi^{+} \mid w(\alpha) \in-\Phi^{+}\right\}\right)$. On notera encore $l$ la fonction sur $N_{\mathbf{G}}(\mathbf{T})$ donnée par $l(\bar{n})$ pour $n \in N_{\mathbf{G}}(\mathbf{T})$. L'ordre de Bruhat sur $W$ associé à $S$ sera noté $\leq$. Si $v$ et $w$ sont deux éléments de $W$, nous écrirons $v<w$ pour dire que $v \leq w$ et $v \neq w$.

Nous noterons $B$ le groupe de tresses associé à $(W, S)$, de générateurs $\left\{\mathbf{s}_{\alpha}\right\}_{\alpha \in \Delta}$. Soit $f: B \rightarrow W$ le morphisme canonique (il vérifie $f\left(\mathbf{s}_{\alpha}\right)=s_{\alpha}$ pour tout $\in \Delta$ ). Il existe alors une et une seule application (qui n'est pas un morphisme de groupes) $\sigma: W \rightarrow B$ vérifiant

- $\sigma(v w)=\sigma(v) \sigma(w)$ si $v$ et $w$ sont deux éléments de $W$ tels que $l(v w)=l(v)+l(w)$;

$-\sigma\left(s_{\alpha}\right)=\mathbf{s}_{\alpha}$ pour tout $\alpha \in \Delta$.

Cette application vérifie $f \circ \sigma=\operatorname{Id}_{W}$.

Nous fixons un morphisme de groupes $\hat{\varphi}: B \rightarrow N_{\hat{\mathbf{G}}}(\hat{\mathbf{T}})$ vérifiant les propriétés suivantes: 
(1) Le diagramme

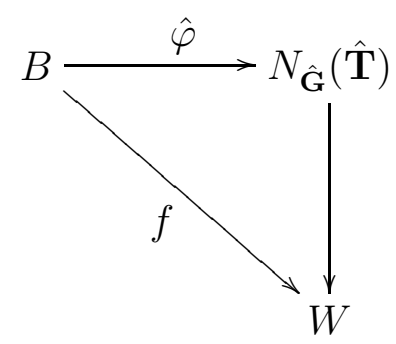

est commutatif. (Dans ce diagramme, la flèche verticale est la surjection canonique.)

(2) Pour tout $\alpha \in \Delta, \hat{\varphi}\left(\mathbf{s}_{\alpha}\right)$ appartient au sous-groupe de $\hat{\mathbf{G}}$ engendré par $\hat{\mathbf{U}}_{\alpha}$ et $\hat{\mathbf{U}}_{-\alpha}$.

L'existence d'un tel morphisme de groupes est assurée par [Ti, §4.6]. Grâce à la propriété (1), il existe un prolongement de $\hat{\varphi}$ en un morphisme de groupes $\hat{\varphi}: B \hat{\mathbf{T}} \rightarrow N_{\hat{\mathbf{G}}}(\hat{\mathbf{T}})$ qui est l'identité sur $\hat{\mathbf{T}}$. Ici, $B \hat{\mathbf{T}}$ désigne le produit semi-direct $B \ltimes \hat{\mathbf{T}}$, l'action de $B$ sur $\hat{\mathbf{T}}$ s'effectuant via le morphisme $f: B \rightarrow W$ et l'action naturelle de $W$ sur $\hat{\mathbf{T}}$. Nous noterons $\varphi: B \mathbf{T} \rightarrow N_{\mathbf{G}}(\mathbf{T})$ le morphisme induit par $\hat{\varphi}$.

Si $w \in W$, posons $\dot{w}=\varphi(\sigma(w))$. Alors, $\dot{w}$ est un représentant de $w$ dans $N_{\mathbf{G}}(\mathbf{T})$. De plus, si $v$ et $w$ sont deux éléments de $W$ tels que $l(v w)=l(v)+l(w)$, alors $\dot{x}=\dot{v} \dot{w}$, où $x=v w$. Remarquons aussi que $\dot{1}=1$. Si $n \in N_{\mathbf{G}}(\mathbf{T})$ et si $w$ désigne sa classe dans $W$, alors il existe un unique élément $t \in \mathbf{T}$ tel que $n=\dot{w} t$. On pose alors $\dot{\sigma}(n)=\sigma(\dot{w}) t \in B \mathbf{T}$.

4.3. Suites d'éléments de $W$. Pour $\mathbf{w}=\left(w_{1}, \ldots, w_{r}\right) \in \Sigma(W)$ (cf $\left.\S 2.1\right)$, nous posons $\dot{\mathbf{w}}=\left(\dot{w}_{1}, \ldots, \dot{w}_{r}\right) \in \Sigma\left(N_{\mathbf{G}}(\mathbf{T})\right)$. Pour $\mathbf{n}=\left(n_{1}, \ldots, n_{r}\right) \in \Sigma\left(N_{\mathbf{G}}(\mathbf{T})\right)$, on pose $\overline{\mathbf{n}}=$ $\left(\bar{n}_{1}, \ldots, \bar{n}_{r}\right)$.

Dans la suite, nous noterons $\sigma: \Sigma(W) \rightarrow B$ et $l: \Sigma(W) \rightarrow(\mathbf{N},+)$ les uniques morphismes de monoïdes prolongeant $\sigma$ et $l$ respectivement. En d'autres termes, $\sigma(\mathbf{w})=$ $\sigma\left(w_{1}\right) \ldots \sigma\left(w_{r}\right)$ et $l(\mathbf{w})=l\left(w_{1}\right)+\cdots+l\left(w_{r}\right)$. Le nombre entier $l(\mathbf{w})$ est appelé la longueur de w. De même, nous noterons $\dot{\sigma}: \Sigma\left(N_{\mathbf{G}}(\mathbf{T})\right) \rightarrow B \mathbf{T}$ l'unique morphisme de monoüdes prolongeant $\dot{\sigma}$.

Si $\mathbf{w}=\left(w_{1}, \ldots, w_{r}\right)$ et $\mathbf{v}=\left(v_{1}, \ldots, v_{r^{\prime}}\right)$ sont deux éléments de $\Sigma(W)$, nous dirons que $\mathbf{v} \leq \mathbf{w}$ si $r=r^{\prime}$ et $v_{i} \leq w_{i}$ pour tout $1 \leq i \leq r$.

Nous noterons encore $\mathbf{w}$ (respectivement $\mathbf{n}$ ) l'automorphisme de $\mathbf{T}$ induit par la conjugaison par $\varphi \sigma(\mathbf{w})$ (respectivement $\varphi \dot{\sigma}(\mathbf{n})$ ). Nous avons ainsi prolongé les actions de $W$ et de $N_{\mathbf{G}}(\mathbf{T})$ sur $\mathbf{T}$ aux monoïdes libres correspondants.

4.4. Tores et caractères. Pour étudier les représentations du groupe $\mathbf{G}^{F}$, nous aurons besoin de quelques résultats de nature combinatoire sur les groupes $\mathbf{T}^{\mathbf{w} F}$. Leur énoncé et leur preuve font l'objet des prochains paragraphes. 
4.4.1. Conjugaison géométrique, séries rationnelles. Nous fixons maintenant un entier naturel non nul $d$ multiple de $\delta$ tel que tout tore maximal $F$-stable de $\mathbf{G}$ soit déployé sur le corps $\mathbf{F}_{q^{d / \delta}}$. En d'autres termes, on a $(w F)^{d}(t)=F^{d}(t)=t^{q^{d / \delta}}$ pour tout $w \in W$ et pour tout $t \in \mathbf{T}$. Il est à noter que $N_{F^{d} / w F}$ induit une surjection $\mathbf{T}^{F^{d}} \longrightarrow \mathbf{T}^{w F}$. De plus, si $t \in \mathbf{T}$ est tel que $N_{F^{d} / w F}(t) \in \mathbf{T}^{w F}$, alors $t \in \mathbf{T}^{F^{d}}$. Pour finir, remarquons que $F^{d}$ agit trivialement sur $W$ et que, pour tout $w \in W$, on a $w F(w) \ldots F^{d-1}(w)=1$.

Soit $\zeta=\imath\left(1 /\left(q^{d / \delta}-1\right)\right)$, où $\imath:(\mathbf{Q} / \mathbf{Z})_{p^{\prime}} \stackrel{\sim}{\longrightarrow} \mathbf{F}^{\times}$est l'isomorphisme choisi dans le $\S 2.2$ $\left(\zeta\right.$ est un générateur du groupe cyclique $\mathbf{F}_{q^{d / \delta}}^{\times}$). Si w est une suite d'éléments de $W$, soit

$$
\begin{array}{ccc}
N_{\mathbf{w}}: Y(\mathbf{T}) & \longrightarrow & \mathbf{T}^{\mathbf{w} F} \\
\lambda & \longmapsto N_{F^{d} / \mathbf{w} F}(\lambda)(\zeta) .
\end{array}
$$

Alors, $N_{\mathbf{w}}$ est un morphisme surjectif de groupes et la suite

$$
0 \longrightarrow Y(\mathbf{T}) \stackrel{\mathbf{w} F-1}{\longrightarrow} Y(\mathbf{T}) \stackrel{N_{\mathbf{w}}}{\longrightarrow} \mathbf{T}^{\mathbf{w} F} 0
$$

est exacte [DiMi, proposition 13.7 (ii)].

Nous noterons $\boldsymbol{\nabla}(\mathbf{T}, W, F)$ (ou $\boldsymbol{\nabla}_{\Lambda}(\mathbf{T}, W, F)$ ) l'ensemble des couples $(\mathbf{w}, \theta)$ où w est une suite finie d'éléments de $W$ et $\theta: \mathbf{T}^{\mathbf{w} F} \rightarrow \Lambda^{\times}$est un caractère linéaire d'ordre inversible dans $\Lambda$.

L'identification de $W$ au groupe de Weyl de $\tilde{\mathbf{G}}$ relatif à $\tilde{\mathbf{T}}\left(c^{\prime}\right.$ est-à-dire $\left.W \stackrel{\sim}{\rightarrow} N_{\tilde{\mathbf{G}}}(\tilde{\mathbf{T}}) / \tilde{\mathbf{T}}\right)$, permet de définir l'application

$$
\begin{aligned}
& \text { Res : } \boldsymbol{\nabla}(\tilde{\mathbf{T}}, W, F) \longrightarrow \nabla(\mathbf{T}, W, F) \\
& (\mathbf{w}, \tilde{\theta}) \longmapsto\left(\mathbf{w}, \operatorname{Res}_{\mathbf{T}^{\mathbf{w} F}}^{\tilde{\mathbf{T}}^{\mathbf{w} F}} \tilde{\theta}\right) .
\end{aligned}
$$

Nous dirons alors que $\operatorname{Res}(\mathbf{w}, \tilde{\theta})$ est la restriction de $(\mathbf{w}, \tilde{\theta})$ à $\mathbf{G}$, ou que $(\mathbf{w}, \tilde{\theta})$ est une extension de $\operatorname{Res}(\mathbf{w}, \hat{\theta})$ à $\tilde{\mathbf{G}}$.

Deux éléments $(\mathbf{w}, \theta)$ et $\left(\mathbf{w}^{\prime}, \theta^{\prime}\right)$ de $\boldsymbol{\nabla}(\mathbf{T}, W, F)$ sont géométriquement conjugués si les caractères linéaires $\theta \circ N_{F^{d} / \mathbf{w} F}$ et $\theta^{\prime} \circ N_{F^{d} / \mathbf{w}^{\prime} F}$ de $\mathbf{T}^{F^{d}}$ sont conjugués sous $W$. D'autre part, les couples $(\mathbf{w}, \theta)$ et $\left(\mathbf{w}^{\prime}, \theta^{\prime}\right)$ appartiennent à la même série rationnelle (ce que nous noterons par $\left.(\mathbf{w}, \theta) \equiv_{W}\left(\mathbf{w}^{\prime}, \theta^{\prime}\right)\right)$ s'il existe des extensions de $(\mathbf{w}, \theta)$ et $\left(\mathbf{w}^{\prime}, \theta^{\prime}\right)$ à $\tilde{\mathbf{G}}$ qui sont géométriquement conjuguées.

Remarque 4.3. Si deux éléments de $\boldsymbol{\nabla}(\mathbf{T}, W, F)$ sont dans la même série rationnelle, alors ils sont géométriquement conjugués. La réciproque n'est pas vraie en général, comme le montre l'exemple du groupe $\mathbf{S L}_{2}(\mathbf{F})$ pour $\mathbf{F}$ de caractéristique différente de 2 .

La réciproque est néanmoins correcte pour $\tilde{\mathbf{G}}$ : deux éléments de $\boldsymbol{\nabla}(\tilde{\mathbf{T}}, W, F)$ sont géométriquement conjugués si et seulement si ils appartiennent à la même série rationnelle. En particulier, la relation d'équivalence $\equiv_{W}$ sur $\boldsymbol{\nabla}(\mathbf{T}, W, F)$ ne dépend pas du choix du groupe réductif $\tilde{\mathbf{G}}$ à centre connexe choisi (voir le théorème 4.8 pour une approche ne faisant pas intervenir $\tilde{\mathbf{G}}$ ). 
4.4.2. Sous-groupes de $\mathbf{T}^{\mathbf{w} F}$. Nous fixons ici, et ce jusqu'à la fin du paragraphe 4.4, quatre suites finies d'éléments de $\bar{S}$,

$$
\mathbf{v}=\left(s_{1}^{\prime}, \ldots, s_{r}^{\prime}\right) \leq \mathbf{x}=\left(t_{1}^{\prime}, \ldots, t_{r}^{\prime}\right) \leq \mathbf{y}=\left(t_{1}, \ldots, t_{r}\right) \leq \mathbf{w}=\left(s_{1}, \ldots, s_{r}\right) .
$$

Soit $1 \leq i \leq r$. Si $s_{i} \neq 1$, nous notons $\alpha_{\mathbf{w}, i}$ l'unique racine simple telle que $s_{i}=s_{\alpha_{\mathbf{w}, i}}$. Lorsque $s_{i}=1$, nous posons $\alpha_{\mathbf{w}, i}=0$.

Soit $I_{\mathbf{w}, \mathbf{v}}=\left\{1 \leq i \leq r \mid s_{i}^{\prime} \neq s_{i}\right\}$. On pose

$$
\alpha_{\mathbf{w}, \mathbf{v}, i}= \begin{cases}\alpha_{\mathbf{w}, i} & \text { si } i \in I_{\mathbf{w}, \mathbf{v}} \\ 0 & \text { sinon. }\end{cases}
$$

On a $\left(s_{i}-s_{i}^{\prime}\right) Y(\mathbf{T}) \subset \mathbf{Z} \alpha_{\mathbf{w}, i}^{\vee}$ pour tout $i$. Par ailleurs, nous posons

$$
\beta_{\mathbf{w}, \mathbf{v}, i}^{\vee}=s_{1} \ldots s_{i-1}\left(\alpha_{\mathbf{w}, \mathbf{v}, i}^{\vee}\right) \text { et } \beta_{\mathbf{w}, i}^{\vee}=s_{1} \ldots s_{i-1}\left(\alpha_{\mathbf{w}, i}^{\vee}\right) \text {. }
$$

Nous allons étudier ici quelques propriétés du sous-réseau $Y_{\mathbf{w}, \mathbf{v}}$ de $Y(\mathbf{T})$ engendré par les $\left(\beta_{\mathbf{w}, \mathbf{v}, i}^{\vee}\right)_{i}$.

Proposition 4.4. Avec les hypothèses et notations ci-dessus, alors

(1) on $a(\mathbf{w}-\mathbf{y}) Y(\mathbf{T}) \subset Y_{\mathbf{w}, \mathbf{v}}$;

(2) le groupe $Y_{\mathbf{w}, \mathbf{v}}$ est engendré par la famille $\left(t_{1} \ldots t_{i-1}\left(\alpha_{\mathbf{w}, \mathbf{v}, i}^{\vee}\right)\right)_{i}$;

(3) on $a(\mathbf{w} F-1) Y(\mathbf{T})+Y_{\mathbf{w}, \mathbf{v}}=(\mathbf{v} F-1) Y(\mathbf{T})+Y_{\mathbf{w}, \mathbf{v}}$;

(4) les morphismes $N_{\mathbf{w}}$ et $N_{\mathbf{y}}$ induisent un isomorphisme

$$
\mathbf{T}^{\mathbf{w} F} / N_{\mathbf{w}}\left(Y_{\mathbf{w}, \mathbf{v}}\right) \simeq \mathbf{T}^{\mathbf{y} F} / N_{\mathbf{y}}\left(Y_{\mathbf{w}, \mathbf{v}}\right) .
$$

Démonstration. Démontrons tout d'abord (1). Puisque $Y_{\mathbf{w}, \mathbf{y}} \subset Y_{\mathbf{w}, \mathbf{v}}$, il suffit de démontrer que $(\mathbf{w}-\mathbf{y}) Y(\mathbf{T}) \subset Y_{\mathbf{w}, \mathbf{y}}$, ce que nous faisons par récurrence sur $m=\left|I_{\mathbf{w}, \mathbf{y}}\right|$.

Si $m=0$, alors $\mathbf{w}=\mathbf{y}$ et le résultat en découle. Supposons maintenant que $m=1$. On a alors $I_{\mathbf{w}, \mathbf{y}}=\{i\}$. On pose $\mathbf{a}=\left(s_{1}, \ldots, s_{i-1}\right), \mathbf{s}=\left(s_{i}\right)$ et $\mathbf{b}=\left(s_{i+1}, \ldots, s_{r}\right)$. Alors,

$$
(\mathbf{w}-\mathbf{y}) Y(\mathbf{T})=(\mathbf{a s b}-\mathbf{a b}) Y(\mathbf{T})=\mathbf{a}((\mathbf{s}-1) Y(\mathbf{T})) \subset \mathbf{a}\left(\mathbf{Z} \alpha_{\mathbf{w}, i}^{\vee}\right)=\mathbf{Z} \beta_{\mathbf{w}, i}^{\vee}=Y_{\mathbf{w}, \mathbf{y}} .
$$

Supposons maintenant que $m \geq 2$ et que le résultat est vrai pour tout couple $\left(\mathbf{w}^{\prime}, \mathbf{y}^{\prime}\right)$ tel que $\mathbf{y}^{\prime} \leq \mathbf{w}^{\prime}$ et $m_{\mathbf{w}^{\prime}, \mathbf{y}^{\prime}}<m$. On écrit

$$
I_{\mathbf{w}, \mathbf{y}}=\left\{i_{1}, \ldots, i_{m}\right\}
$$

où $i_{1}<\cdots<i_{m}$. On note $\mathbf{y}^{\prime}$ l'unique suite telle que $\mathbf{y}^{\prime} \leq \mathbf{w}$ et

$$
I_{\mathbf{w}, \mathbf{y}^{\prime}}=\left\{i_{2}, \ldots, i_{m}\right\}
$$

On a

$$
(\mathbf{w}-\mathbf{y}) Y(\mathbf{T}) \subset\left(\mathbf{w}-\mathbf{y}^{\prime}\right) Y(\mathbf{T})+\left(\mathbf{y}^{\prime}-\mathbf{y}\right) Y(\mathbf{T})
$$

Or, par hypothèse de récurrence, $\left(\mathbf{w}-\mathbf{y}^{\prime}\right) Y(\mathbf{T}) \subset Y_{\mathbf{w}, \mathbf{y}^{\prime}}$ et $\left(\mathbf{y}^{\prime}-\mathbf{y}\right) Y(\mathbf{T}) \subset Y_{\mathbf{y}^{\prime}, \mathbf{y}}$. Le choix que nous avons fait pour $\mathbf{y}^{\prime}$ implique que $\beta_{\mathbf{y}^{\prime}, i_{1}}^{\vee}=\beta_{\mathbf{w}, i_{1}}^{\vee}$, donc $Y_{\mathbf{w}, \mathbf{y}^{\prime}}+Y_{\mathbf{y}^{\prime}, \mathbf{y}}=Y_{\mathbf{w}, \mathbf{y}}$. Cela termine la démonstration de (1). 
Démontrons maintenant (2). Il suffit de démontrer que, pour tout $i \in I_{\mathbf{w}, \mathbf{v}}$, il existe une famille $\left(\mu_{j}\right)_{j \in I_{\mathbf{w}, \mathbf{v}}, j<i}$ d'entiers tels que

$$
t_{1} \ldots t_{i-1}\left(\alpha_{\mathbf{w}, i}^{\vee}\right)=\beta_{\mathbf{w}, i}^{\vee}+\sum_{\substack{j \in I_{\mathbf{w}, \mathbf{v}} \\ j<i}} \mu_{j} \beta_{\mathbf{w}, j}^{\vee}
$$

c'est-à-dire tels que

$$
\left(s_{1} \ldots s_{i-1}-t_{1} \ldots t_{i-1}\right)\left(\alpha_{\mathbf{w}, i}^{\vee}\right)=-\sum_{\substack{j \in I_{\mathbf{w}, \mathbf{v}} \\ j<i}} \mu_{j} \beta_{\mathbf{w}, j}^{\vee}
$$

Cela résulte de (1) appliqué dans le cas où $\mathbf{w}=\left(s_{1}, \ldots, s_{i-1}\right)$ et $\mathbf{y}=\mathbf{v}=\left(t_{1}, \ldots, t_{i-1}\right)$.

Pour finir la démonstration de la proposition 4.4, il suffit de remarquer que (3) découle immédiatement de (1) tandis que (4) résulte de (3) et de la suite exacte 4.2.

Corollaire 4.5. On a $Y_{\mathbf{w}, \mathbf{v}}=Y_{\mathbf{w}, \mathbf{y}}+Y_{\mathbf{y}, \mathbf{v}}$.

Démonstration. On a $I_{\mathbf{w}, \mathbf{v}}=I_{\mathbf{w}, \mathbf{y}} \amalg I_{\mathbf{y}, \mathbf{v}}$. Tout d'abord, $Y_{\mathbf{y}, \mathbf{v}}=\sum_{i \in I_{\mathbf{y}, \mathbf{v}}} \mathbf{Z} t_{1} \cdots t_{i-1}\left(\alpha_{\mathbf{w}, i}^{\vee}\right)$ car $\beta_{\mathbf{y}, i}^{\vee}=t_{1} \cdots t_{i-1}\left(\alpha_{\mathbf{w}, i}^{\vee}\right)$ pour $i \in I_{\mathbf{y}, \mathbf{v}}$. La proposition 4.4 (2) appliquée au cas $\mathbf{v}=$ $\mathbf{y}$ montre que $Y_{\mathbf{w}, \mathbf{y}}=\sum_{i \in I_{\mathbf{w}, \mathbf{y}}} \mathbf{Z} t_{1} \cdots t_{i-1}\left(\alpha_{\mathbf{w}, i}^{\vee}\right)$. Enfin, $Y_{\mathbf{w}, \mathbf{v}}=\sum_{i \in I_{\mathbf{w}, \mathbf{v}}} \mathbf{Z} t_{1} \cdots t_{i-1}\left(\alpha_{\mathbf{w}, i}^{\vee}\right)$ d'après la proposition $4.4(2)$.

Corollaire 4.6. Soit $\theta$ un caractère linéaire de $\mathbf{T}^{\mathbf{w} F}$ qui est trivial sur $N_{\mathbf{w}}\left(Y_{\mathbf{w}, \mathbf{v}}\right)$. Notons $\theta_{\mathbf{y}}$ le caractère linéaire de $\mathbf{T}^{\mathbf{y} F}$ trivial sur $N_{\mathbf{y}}\left(Y_{\mathbf{w}, \mathbf{v}}\right)$ défini via l'isomorphisme de la proposition 4.4 (4) (c'est-à-dire tel que $\left.\theta_{\mathbf{y}} \circ N_{\mathbf{y}}=\theta \circ N_{\mathbf{w}}\right)$. Alors, les paires $(\mathbf{w}, \theta)$ et $\left(\mathbf{y}, \theta_{\mathbf{y}}\right)$ sont dans la même série rationnelle.

Démonstration. Pour démontrer le corollaire 4.6, on peut se ramener au cas où $\mathbf{G}=\tilde{\mathbf{G}}$ : c'est alors immédiat.

Fixons maintenant un caractère linéaire $\theta: \mathbf{T}^{\mathbf{w} F} \rightarrow \Lambda^{\times}$et notons $\mathcal{I}(\mathbf{w}, \theta)$ l'ensemble des $\mathbf{w}^{\prime} \leq \mathbf{w}$ tels que $\theta$ est trivial sur $N_{\mathbf{w}}\left(Y_{\mathbf{w}, \mathbf{w}^{\prime}}\right)$. L'ensemble $\mathcal{I}(\mathbf{w}, \theta)$ admet un plus petit élément, noté $\mathbf{w}_{\theta}=\left(s_{1, \theta}, \ldots, s_{r, \theta}\right)$, qui est défini par

$$
s_{i, \theta}= \begin{cases}1 & \text { si } \theta\left(N_{\mathbf{w}}\left(\beta_{\mathbf{w}, i}^{\vee}\right)\right)=1 \\ s_{i} & \text { sinon. }\end{cases}
$$

On a donc

$$
\mathcal{I}(\mathbf{w}, \theta)=\left\{\mathbf{x} \in \Sigma(\bar{S}) \mid \mathbf{w}_{\theta} \leq \mathbf{x} \leq \mathbf{w}\right\}
$$

D'après la proposition $4.4(4)$, si $\mathbf{y} \in \mathcal{I}(\mathbf{w}, \theta)$, alors $\theta$ définit un caractère linéaire $\theta_{\mathbf{y}}$ de $\mathbf{T}^{\mathbf{y} F}$ trivial sur $N_{\mathbf{y}}\left(Y_{\mathbf{w}, \mathbf{w}_{\theta}}\right)$. Notons que $\theta_{\mathbf{w}}=\theta$.

Corollaire 4.7. Supposons que $\mathbf{y} \in \mathcal{I}(\mathbf{w}, \theta)$. Alors

$$
\mathcal{I}\left(\mathbf{y}, \theta_{\mathbf{y}}\right)=\left\{\mathbf{w}^{\prime} \in \mathcal{I}(\mathbf{w}, \theta) \mid \mathbf{w}_{\theta} \leq \mathbf{w}^{\prime} \leq \mathbf{y}\right\}
$$

En d'autres termes, $\mathbf{y}_{\theta_{\mathbf{y}}}=\mathbf{w}_{\theta}$. 
Démonstration. Pour tout $\mathbf{x} \leq \mathbf{y}$, on a

$$
\theta \circ N_{\mathbf{w}}\left(Y_{\mathbf{w}, \mathbf{x}}\right)=\theta \circ N_{\mathbf{w}}\left(Y_{\mathbf{w}, \mathbf{y}}+Y_{\mathbf{y}, \mathbf{x}}\right)
$$

d'après le corollaire 4.5. Or, $\theta \circ N_{\mathbf{w}}\left(Y_{\mathbf{w}, \mathbf{y}}\right)=1$ car $\mathbf{y} \in \mathcal{I}(\mathbf{w}, \theta)$. Enfin, $\theta \circ N_{\mathbf{w}}=\theta_{\mathbf{y}} \circ N_{\mathbf{y}}$, ce qui montre que

$$
\theta \circ N_{\mathbf{w}}\left(Y_{\mathbf{w}, \mathbf{x}}\right)=\theta_{\mathbf{y}} \circ N_{\mathbf{y}}\left(Y_{\mathbf{y}, \mathbf{x}}\right),
$$

donc que $\mathbf{x} \in \mathcal{I}(\mathbf{w}, \theta)$ si et seulement si $\mathbf{x} \in \mathcal{I}\left(\mathbf{y}, \theta_{\mathbf{y}}\right)$.

On dit que $(\mathbf{w}, \theta),\left(\mathbf{w}^{\prime}, \theta^{\prime}\right) \in \boldsymbol{\nabla}(\mathbf{T}, W, F)$ sont élémentairement équivalents si l'une des deux conditions suivantes est réalisée :

1. il existe $v \in W$ tel que $f\left(\sigma\left(\mathbf{w}^{\prime}\right)\right)=v f(\sigma(\mathbf{w})) F(v)^{-1}$ et $\theta=\theta^{\prime} \circ v(W$-conjugaison $)$

2. $\mathbf{w} \in \Sigma(S), \mathbf{w}^{\prime}=\mathbf{w}_{\theta}$ et $\theta^{\prime}=\theta_{\mathbf{w}_{\theta}}$.

On définit la relation d'équivalence $\sim_{W}$ sur $\boldsymbol{\nabla}(\mathbf{T}, W, F)$ comme la clôture transitive (et symétrique) de la relation d'équivalence élémentaire.

Le théorème suivant fournit une nouvelle caractérisation de la conjugaison rationnelle de caractères de tores, qui ne fait pas intervenir de groupe $\tilde{\mathbf{G}}$.

Théorème 4.8. Deux couples $(\mathbf{w}, \theta)$ et $\left(\mathbf{w}^{\prime}, \theta^{\prime}\right)$ sont $\sim_{W}$-équivalents si et seulement si ils sont dans la même série rationnelle.

Démonstration. Grâce au corollaire 4.6 , il est clair que la relation $\sim_{W}$ est plus fine que la relation $\equiv_{W}$. Par conséquent, le théorème 4.8 est conséquence du lemme suivant.

Lemme 4.9. Soient $w, w^{\prime} \in W$ et $((w), \theta)$ et $\left(\left(w^{\prime}\right), \theta^{\prime}\right)$ deux éléments de $\boldsymbol{\nabla}(\mathbf{T}, W, F)$ vérifiant les propriétés suivantes:

(1) $((w), \theta)$ et $\left(\left(w^{\prime}\right), \theta^{\prime}\right)$ sont dans la même série rationnelle;

$(2)((w), \theta)$ et $\left(\left(w^{\prime}\right), \theta^{\prime}\right)$ sont minimaux (pour les longueurs de $(w)$ et $\left.\left(w^{\prime}\right)\right)$ dans leurs classes respectives pour la relation $\sim_{W}$.

Alors, $((w), \theta)$ et $\left(\left(w^{\prime}\right), \theta^{\prime}\right)$ sont conjugués sous $W$.

Démonstration. Tout comme dans le corollaire 4.6, on peut se ramener au cas où le centre de $\mathbf{G}$ est connexe. Posons

$$
\Phi_{w, \theta}^{\vee}=\left\{\alpha^{\vee} \in \Phi^{\vee} \mid \theta \circ N_{w}\left(\alpha^{\vee}\right)=1\right\} \quad \text { et } \quad \Phi_{w, \theta}^{\vee+}=\Phi_{w, \theta}^{\vee} \cap\left\{\alpha^{\vee} \mid \alpha \in \Phi^{+}\right\} .
$$

Le sous-ensemble $\Phi_{w, \theta}^{\vee}$ de $\Phi^{\vee}$ est clos et symétrique. C'est un donc un sous-système de $\Phi^{\vee}$ dont $\Phi_{w, \theta}^{\vee+}$ est un système de coracines positives. On note $W_{w, \theta}$ le groupe engendré par les $s_{\alpha}$ avec $\alpha^{\vee} \in \Phi_{w, \theta}^{\vee}$. C'est aussi le stabilisateur de $\theta \circ N_{w}$ (cela résulte de la connexité du centre de $\mathbf{G}$ et de [DeLu, théorème 5.13]).

Il est clair que $w \phi$ stabilise $\Phi_{w, \theta}^{\vee}$. Nous allons montrer que l'hypothèse (2) implique que $w \phi$ stabilise $\Phi_{w, \theta}^{\vee+}$. Pour cela, choisissons une décomposition réduite $\left(s_{1}, \ldots, s_{r}\right)$ de $w$ où les $s_{i}$ sont dans $S$ et notons $\alpha_{i}$ la racine simple associée à $s_{i}$. Alors les $s_{1} \ldots s_{i-1}\left(\alpha_{i}^{\vee}\right)$ sont les coracines positives rendues négatives par l'action de $w^{-1}$. Par minimalité de $((w), \theta)$, on a $\left(s_{1}, \ldots, s_{r}\right)_{\theta}=\left(s_{1}, \ldots, s_{r}\right)$. Donc $s_{1} \ldots s_{i-1}\left(\alpha_{i}^{\vee}\right)$ n'appartient pas à $\Phi_{w, \theta}^{\vee}$. Cela montre que $\phi^{-1} w^{-1}\left(\Phi_{w, \theta}^{\vee+}\right) \subset \Phi^{+}$. Finalement, on a bien que $w \phi$ stabilise $\Phi_{w, \theta}^{\vee+}$. 
D'autre part, (1) montre qu'il existe $x \in W$ tel que $\theta \circ N_{w}=\theta^{\prime} \circ N_{w^{\prime}} \circ x$. Par conséquent $x\left(\Phi_{w, \theta}^{\vee}\right)=\Phi_{w^{\prime}, \theta^{\prime}}^{\vee}$, donc il existe un élément $a \in W_{w^{\prime}, \theta^{\prime}}$ tel que $a x\left(\Phi_{w, \theta}^{\vee+}\right)=\Phi_{w^{\prime}, \theta^{\prime}}^{\vee}$. Or, $\Phi_{w, \theta}^{\vee+}$ est $w \phi$-stable, et $\Phi_{w^{\prime}, \theta^{\prime}}^{\vee+}$ est $w^{\prime} \phi$-stable. Donc axwF $(a x)^{-1} \phi \phi^{-1} w^{\prime-1}=a x w F(a x)^{-1} w^{\prime-1}$ stabilise $\Phi_{w^{\prime}, \theta^{\prime}}^{\vee+}$

Or, $\theta^{\prime} \circ N_{w^{\prime}} \circ \operatorname{axwF}(a x)^{-1} w^{\prime-1}=\theta \circ N_{w} \circ w F(a x)^{-1} w^{\prime-1}$. Soit $\lambda \in Y(\mathbf{T})$. Alors

$$
\begin{aligned}
& \theta \circ N_{F^{d} / w F}\left(w F(a x)^{-1} w^{-1} \lambda(\zeta)\right)=\theta \circ N_{F^{d} / w F}\left(w F \left({ }^{\left.\left.(a x)^{-1} F^{-1} w^{-1} \lambda(\zeta)\right)\right)}\right.\right. \\
& =\theta \circ N_{F^{d} / w F}\left({ }^{\left.(a x)^{-1} F^{-1} w^{\prime-1} \lambda(\zeta)\right)}\right. \\
& =\theta^{\prime} \circ N_{w^{\prime}}\left(F^{-1} w^{-1} \lambda(\zeta)\right) \\
& =\theta^{\prime} \circ N_{w^{\prime}}(\lambda(\zeta)) \text {. }
\end{aligned}
$$

Ici, $F^{-1}$ est vu comme un automorphisme du groupe T. Ceci montre que $\operatorname{axw} F(a x)^{-1} w^{-1}$ est dans $W_{w^{\prime}, \theta^{\prime}}$. Puisqu'il stabilise $\Phi_{w^{\prime}, \theta^{\prime}}^{\vee+}$, il est trivial. Par conséquent, on a $w^{\prime}=a x w F(a x)^{-1}$. En outre, $\theta^{\prime}=\theta \circ$ ax car la norme est surjective.

Remarque 4.10. La preuve montre aussi qu'un couple minimal correspond à un couple maximalement déployé au sens de Deligne et Lusztig [DeLu, définition 5.25]. Lorsque le centre de $\mathbf{G}$ est connexe, il est démontré dans [DeLu, proposition 5.26] que deux couples $((w), \theta)$ et $\left(\left(w^{\prime}\right), \theta^{\prime}\right)$ appartenant à une même série rationnelle et maximalement déployés sont conjugués sous $W$, cas particulier du lemme 4.9.

4.4.3. Une application. L'objet de ce paragraphe est la définition et l'étude d'un groupe diagonalisable qui interviendra dans la construction de recollements de variétés de DeligneLusztig au $\S 6$.

Fixons tout d'abord quelques notations. Définissons

$\mathbf{S}_{\mathbf{w}, \mathbf{v}}=\left\{\left(a_{1}, \ldots, a_{r}\right) \in \mathbf{T}^{r} \mid a_{i}^{-1} s_{i} a_{i+1} \in \operatorname{Im} \alpha_{\mathbf{w}, \mathbf{v}, i}^{\vee}\right.$ pour $1 \leq i \leq r-1$ et $\left.a_{r}^{-1} s_{r} F a_{1} \in \operatorname{Im} \alpha_{\mathbf{w}, \mathbf{v}, r}^{\vee}\right\}$ et

$$
\begin{aligned}
\mu_{\mathbf{y}}: \mathbf{T} & \longrightarrow \\
a & \longmapsto\left(a,{ }^{t_{1}} a, \ldots,{ }^{t_{r-1} \ldots t_{1}} a\right) .
\end{aligned}
$$

Proposition 4.11. Avec les notations ci-dessus, on a:

(1) $\mu_{\mathbf{y}}\left(\mathbf{T}^{\mathbf{y} F}\right) \subset \mathbf{S}_{\mathbf{w}, \mathbf{v}}$.

(2) $\mathbf{S}_{\mathbf{w}, \mathbf{v}}=\mu_{\mathbf{y}}\left(\mathbf{T}^{\mathbf{y} F}\right) \cdot \mathbf{S}_{\mathbf{w}, \mathbf{v}}^{\circ}$.

(3) $\mu_{\mathbf{y}}\left(N_{\mathbf{y}}\left(Y_{\mathbf{w}, \mathbf{v}}\right)\right) \subset \mathbf{S}_{\mathbf{w}, \mathbf{v}}^{\circ}$ et le diagramme suivant est commutatif

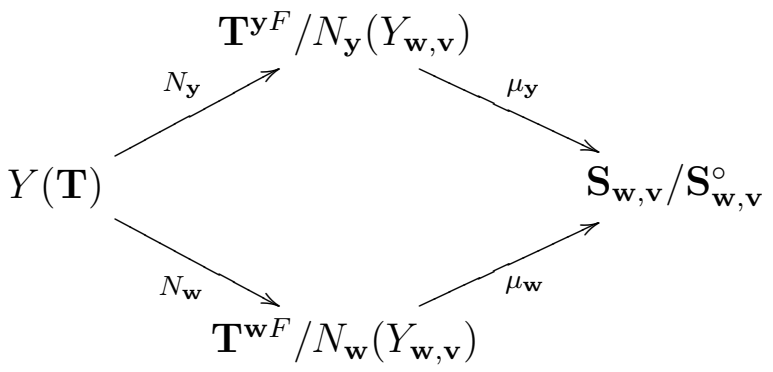

(4) Si les coracines de $\mathbf{G}$ sont injectives, alors $\mu_{\mathbf{y}}\left(\mathbf{T}^{\mathbf{y} F}\right) \cap \mathbf{S}_{\mathbf{w}, \mathbf{v}}^{\circ}=\mu_{\mathbf{y}}\left(N_{\mathbf{y}}\left(Y_{\mathbf{w}, \mathbf{v}}\right)\right)$. 
Démonstration. Posons $\mathbf{G}^{\prime}=\mathbf{G}^{r}, \mathbf{T}^{\prime}=\mathbf{T}^{r}$ et $W^{\prime}=W^{r}$. Dans cette preuve, les suites $\mathbf{v}$, $\mathbf{y}$ et $\mathbf{w}$ seront vues comme des éléments de $W^{\prime}$. Posons d'autre part

$$
\mathbf{T}_{\mathbf{w}, \mathbf{v}}^{\prime}=\operatorname{Im} \alpha_{\mathbf{w}, \mathbf{v}, 1}^{\vee} \times \cdots \times \operatorname{Im} \alpha_{\mathbf{w}, \mathbf{v}, r}^{\vee} .
$$

On a alors

$$
{ }^{\mathbf{w}_{t}} \mathbf{y}^{-1} \in \mathbf{T}_{\mathbf{w}, \mathbf{v}}^{\prime}
$$

pour tout $t \in \mathbf{T}^{\prime}$. Pour finir, notons

$$
\begin{aligned}
& F^{\prime}: \quad \mathbf{G}^{\prime} \quad \longrightarrow \quad \mathbf{G}^{\prime} \\
& \left(g_{1}, \ldots, g_{r}\right) \longmapsto\left(g_{2}, \ldots, g_{r}, F\left(g_{1}\right)\right) .
\end{aligned}
$$

Alors $F^{\prime r \delta}$ est un endomorphisme de Frobenius sur $\mathbf{G}^{\prime}$ qui munit ce dernier d'une structure rationnelle sur $\mathbf{F}_{q}$. Avec ces notations, le groupe diagonalisable $\mathbf{S}_{\mathbf{w}, \mathbf{v}}$ peut être défini ainsi :

$$
\mathbf{S}_{\mathbf{w}, \mathbf{v}}=\left\{t \in \mathbf{T}^{\prime} \mid t^{-1} \mathbf{w} F^{\prime} t \in \mathbf{T}_{\mathbf{w}, \mathbf{v}}^{\prime}\right\}
$$

D'après $(*)$, cette caractérisation est équivalente à la suivante :

$$
\mathbf{S}_{\mathbf{w}, \mathbf{v}}=\left\{t \in \mathbf{T}^{\prime} \mid t^{-1} \mathbf{y} F^{\prime} t \in \mathbf{T}_{\mathbf{w}, \mathbf{v}}^{\prime}\right\}
$$

Compte tenu de cette dernière définition, on a $\mathbf{T}^{\prime \mathbf{y} F^{\prime}} \subset \mathbf{S}_{\mathbf{w}, \mathbf{v}}$ et $\mathbf{S}_{\mathbf{w}, \mathbf{v}}=\mathbf{T}^{\prime \mathbf{y} F^{\prime}} \cdot \mathbf{S}_{\mathbf{w}, \mathbf{v}}^{\circ}$ (voir remarque 4.1). Or, un calcul facile montre que $\mathbf{T}^{\mathbf{y} F^{\prime}}=\mu_{\mathbf{y}}\left(\mathbf{T}^{\mathbf{y} F}\right)$, ce qui termine la preuve de (1) et (2).

Démontrons maintenant (3). On a, pour tout $w^{\prime} \in W^{\prime},\left(w^{\prime} F^{\prime}\right)^{r d}=F^{\prime r d}$, et $F^{\prime r d}$ est un endomorphisme de Frobenius déployé sur $\mathbf{T}^{\prime}$. On définit alors

$$
\begin{aligned}
& N_{w^{\prime}}^{\prime}: Y\left(\mathbf{T}^{\prime}\right) \quad \longrightarrow \quad \mathbf{T}^{\prime w^{\prime} F^{\prime}} \\
& \lambda \longmapsto N_{F^{\prime r d} / w^{\prime} F^{\prime}}(\lambda(\zeta)) .
\end{aligned}
$$

Remarquons tout d'abord que $N_{F^{\prime r d} / \mathbf{y} F^{\prime}}\left(\mathbf{T}_{\mathbf{w}, \mathbf{v}}^{\prime}\right)$ est contenu dans $\mathbf{S}_{\mathbf{w}, \mathbf{v}}$ car $\mathbf{y} F^{\prime} \circ N_{F^{\prime r d} / \mathbf{y} F^{\prime}}=$ $F^{\prime r d}$ est déployé donc stabilise $\mathbf{T}_{\mathbf{w}, \mathbf{v}}^{\prime}$. Puisque $N_{F^{\prime r d} / \mathbf{y} F^{\prime}}\left(\mathbf{T}_{\mathbf{w}, \mathbf{v}}^{\prime}\right)$ est connexe, il est en fait contenu dans $\mathbf{S}_{\mathbf{w}, \mathbf{v}}^{\circ}$. D'autre part, le noyau de $N_{F^{\prime r d} / \mathbf{y} F^{\prime}}$ étant fini (il est contenu dans $\mathbf{T}^{\prime F^{\prime r d}}$, on a

Par conséquent,

$$
\operatorname{dim} \mathbf{T}_{\mathbf{w}, \mathbf{v}}^{\prime}=\operatorname{dim} N_{F^{\prime r d} / \mathbf{y} F^{\prime}}\left(\mathbf{T}_{\mathbf{w}, \mathbf{v}}^{\prime}\right)=\operatorname{dim} \mathbf{S}_{\mathbf{w}, \mathbf{v}}
$$

$$
\mathbf{S}_{\mathbf{w}, \mathbf{v}}^{\circ}=N_{F^{\prime r d} / \mathbf{y} F^{\prime}}\left(\mathbf{T}_{\mathbf{w}, \mathbf{v}}^{\prime}\right)
$$

Ce résultat étant vrai pour tout $\mathbf{y}$ compris entre $\mathbf{v}$ et $\mathbf{w}$, on a aussi

$$
\mathbf{S}_{\mathbf{w}, \mathbf{v}}^{\circ}=N_{F^{\prime r d} / \mathbf{w} F^{\prime}}\left(\mathbf{T}_{\mathbf{w}, \mathbf{v}}^{\prime}\right) \text {. }
$$

Pour simplifier les notations, posons $Y_{\mathbf{w}, \mathbf{v}}^{\prime}=Y\left(\mathbf{T}_{\mathbf{w}, \mathbf{v}}^{\prime}\right)$. C'est un sous-réseau facteur direct de $Y\left(\mathbf{T}^{\prime}\right)$. On a alors, d'après (a),

$$
Y\left(\mathbf{S}_{\mathbf{w}, \mathbf{v}}^{\circ}\right)=\left(\mathbf{Q} \otimes_{\mathbf{z}} N_{F^{\prime r d} / \mathbf{y} F^{\prime}}\left(Y_{\mathbf{w}, \mathbf{v}}^{\prime}\right)\right) \cap Y\left(\mathbf{T}^{\prime}\right) .
$$

De même, d'après (a'), on a

$$
Y\left(\mathbf{S}_{\mathbf{w}, \mathbf{v}}^{\circ}\right)=\left(\mathbf{Q} \otimes_{\mathbf{z}} N_{F^{\prime r d} / \mathbf{w} F^{\prime}}\left(Y_{\mathbf{w}, \mathbf{v}}^{\prime}\right)\right) \cap Y\left(\mathbf{T}^{\prime}\right) .
$$


L'égalité (b) montre en particulier que

(c)

$$
N_{\mathbf{y}}^{\prime}\left(Y_{\mathbf{w}, \mathbf{v}}^{\prime}\right) \subset \mathbf{S}_{\mathbf{w}, \mathbf{v}}^{\circ}
$$

De même (b') montre que

$$
N_{\mathbf{w}}^{\prime}\left(Y_{\mathbf{w}, \mathbf{v}}^{\prime}\right) \subset \mathbf{S}_{\mathbf{w}, \mathbf{v}}^{\circ}
$$

D'autre part, si $\lambda^{\prime} \in Y\left(\mathbf{T}^{\prime}\right)$, alors il existe $\mu^{\prime} \in \mathbf{Q} \otimes_{\mathbf{z}} Y\left(\mathbf{T}^{\prime}\right)$ tel que $\lambda^{\prime}=\left(\mathbf{w} F^{\prime}-1\right)\left(\mu^{\prime}\right)$. Par suite,

$$
\begin{aligned}
& N_{F^{\prime r d} / \mathbf{w} F^{\prime}}\left(\lambda^{\prime}\right)-N_{F^{\prime r d} / \mathbf{y} F^{\prime}}\left(\lambda^{\prime}\right) \\
= & N_{F^{\prime r d} / \mathbf{w} F^{\prime}} \circ\left(\mathbf{w} F^{\prime}-1\right)\left(\mu^{\prime}\right)-N_{F^{\prime r d} / \mathbf{y} F^{\prime}} \circ\left(\mathbf{y} F^{\prime}-1\right)\left(\mu^{\prime}\right)-N_{F^{\prime r d} / \mathbf{y} F^{\prime}} \circ(\mathbf{y}-\mathbf{w}) \circ F^{\prime}\left(\mu^{\prime}\right) \\
= & \left(q^{d / \delta}-1\right) \mu^{\prime}-\left(q^{d / \delta}-1\right) \mu^{\prime}-N_{F^{\prime r d} / \mathbf{y} F^{\prime}} \circ(\mathbf{y}-\mathbf{w}) \circ F^{\prime}\left(\mu^{\prime}\right) \\
= & -N_{F^{\prime r d} / \mathbf{y} F^{\prime}} \circ(\mathbf{y}-\mathbf{w}) \circ F^{\prime}\left(\mu^{\prime}\right) .
\end{aligned}
$$

Or, $(\mathbf{y}-\mathbf{w}) \circ F^{\prime}\left(\mu^{\prime}\right) \in \mathbf{Q} \otimes_{\mathbf{z}} Y_{\mathbf{w}, \mathbf{v}}^{\prime}$, donc

$$
N_{F^{\prime r d} / \mathbf{w} F^{\prime}}\left(\lambda^{\prime}\right)-N_{F^{\prime \prime d} / \mathbf{y} F^{\prime}}\left(\lambda^{\prime}\right) \in\left(\mathbf{Q} \otimes_{\mathbf{Z}} N_{F^{\prime r d} / \mathbf{y} F^{\prime}}\left(Y_{\mathbf{w}, \mathbf{v}}^{\prime}\right)\right) \cap Y\left(\mathbf{T}^{\prime}\right)=Y\left(\mathbf{S}_{\mathbf{w}, \mathbf{v}}^{\circ}\right) .
$$

Cela montre que le diagramme

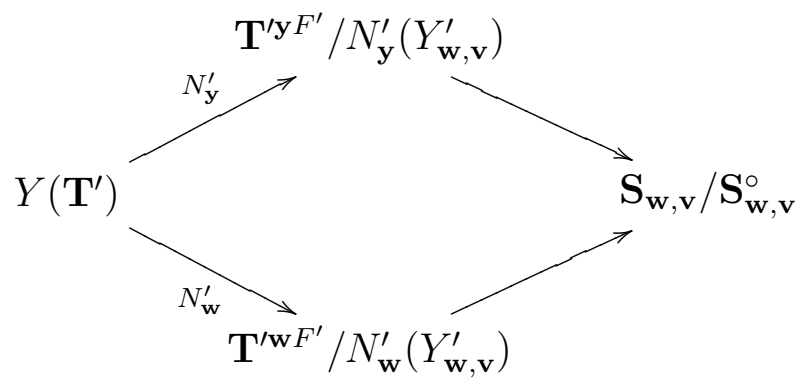

est commutatif.

Pour déduire (3), il faut encore comparer $\mu_{\mathbf{y}} \circ N_{\mathbf{y}}$ et $N_{\mathbf{y}}^{\prime}$. Soit $p_{1}$ la première projection $\mathbf{T}^{\prime} \rightarrow \mathbf{T}$. On a

$$
p_{1} \circ N_{\mathbf{y}}^{\prime}(0, \ldots, 0, \lambda, 0, \ldots, 0)=N_{F^{d} / \mathbf{y} F}\left(t_{1} \cdots t_{i-1}(\lambda)(\zeta)\right)=N_{\mathbf{y}}\left(t_{1} \cdots t_{i-1}(\lambda)\right),
$$

où le terme $\lambda$ a été placé en $i$-ème position. Puisque $p_{1} \circ \mu_{\mathbf{y}}$ est l'identité, on a

$$
p_{1} \circ \mu_{\mathbf{y}} \circ N_{\mathbf{y}}\left(t_{1} \ldots t_{i-1}(\lambda)\right)=p_{1} \circ N_{\mathbf{y}}^{\prime}(0, \ldots, 0, \lambda, 0, \ldots, 0) .
$$

Or, $N_{\mathbf{y}}^{\prime}(0, \ldots, 0, \lambda, 0, \ldots, 0) \in \mathbf{T}^{\prime \mathbf{y} F^{\prime}}=\mu_{\mathbf{y}}\left(\mathbf{T}^{\mathbf{y} F}\right)$, donc

$$
\mu_{\mathbf{y}} \circ N_{\mathbf{y}}\left(t_{1} \ldots t_{i-1}(\lambda)\right)=N_{\mathbf{y}}^{\prime}(0, \ldots, 0, \lambda, 0, \ldots, 0) .
$$

L'inclusion $\mu_{\mathbf{y}}\left(N_{\mathbf{y}}\left(Y_{\mathbf{w}, \mathbf{v}}\right)\right) \subset \mathbf{S}_{\mathbf{w}, \mathbf{v}}^{\circ}$ découle de (c), (d) et de la proposition 4.4, (2). La commutativité du diagramme dans (3) résulte de la commutativité du diagramme précédent et de (d).

Passons enfin à (4). Compte tenu de (a), (d) et de la proposition 4.4, (2), il suffit de montrer que

$$
N_{F^{\prime r d} / \mathbf{y} F^{\prime}}\left(\mathbf{T}_{\mathbf{w}, \mathbf{v}}^{\prime}\right) \cap \mathbf{T}^{\prime \mathbf{y} F^{\prime}}=N_{\mathbf{y}}^{\prime}\left(Y_{\mathbf{w}, \mathbf{v}}^{\prime}\right) .
$$


Or, si $t \in \mathbf{T}^{\prime}, N_{F^{\prime r d} / \mathbf{y} F^{\prime}}(t) \in \mathbf{T}^{\prime \mathbf{y} F^{\prime}}$ si et seulement si $t \in \mathbf{T}^{\prime F^{\prime r d}}$. Par conséquent,

$$
N_{F^{\prime r d} / \mathbf{y} F^{\prime}}\left(\mathbf{T}_{\mathbf{w}, \mathbf{v}}^{\prime}\right) \cap \mathbf{T}^{\prime \mathbf{y} F^{\prime}}=N_{F^{\prime r d} / \mathbf{y} F^{\prime}}\left(\mathbf{T}_{\mathbf{w}, \mathbf{v}}^{\prime F^{\prime r d}}\right) .
$$

Il suffit donc de prouver que

$$
\mathbf{T}_{\mathbf{w}, \mathbf{v}}^{F^{\prime r d}}=\left\{\lambda(\zeta) \mid \lambda \in Y_{\mathbf{w}, \mathbf{v}}^{\prime}\right\}
$$

Mais

$$
\mathbf{T}_{\mathbf{w}, \mathbf{v}}^{F^{\prime r d}}=\left(\operatorname{Im} \alpha_{\mathbf{w}, \mathbf{v}, 1}^{\vee}\right)^{F^{d}} \times \cdots \times\left(\operatorname{Im} \alpha_{\mathbf{w}, \mathbf{v}, r}^{\vee}\right)^{F^{d}} .
$$

Puisque les coracines de $\mathbf{G}$ sont injectives et que l'endomorphisme $F^{d}$ de $\mathbf{T}$ est un endomorphisme de Frobenius déployé sur $\mathbf{F}_{q^{d / \delta}}$, on a

$$
\left(\operatorname{Im} \alpha_{\mathbf{w}, \mathbf{v}, i}^{\vee}\right)^{F^{d}}=\alpha_{\mathbf{w}, \mathbf{v}, i}^{\vee}\left(\mathbf{F}_{q^{d / \delta}}^{\times}\right)
$$

pour $1 \leq i \leq r$. Cela montre que

$$
\mathbf{T}_{\mathbf{w}, \mathbf{v}}^{F^{\prime r d}}=\alpha_{\mathbf{w}, \mathbf{v}, 1}^{\vee}\left(\mathbf{F}_{q^{d / \delta}}^{\times}\right) \times \cdots \times \alpha_{\mathbf{w}, \mathbf{v}, r}^{\vee}\left(\mathbf{F}_{q^{d / \delta}}^{\times}\right) .
$$

La preuve du (4) est maintenant complète.

Remarque 4.12. La proposition précédente donne une autre construction de l'isomorphisme de la proposition 4.4 (4), montrant au passage qu'elle est indépendante des choix des isomorphismes de $\S 2.2$.

\section{VARiÉtÉS DE Deligne-Lusztig GÉnÉRAlisÉES}

5.1. Comparaison de modèles. Nous commençons cette partie par un résultat géométrique concernant une classe de variétés qui contient les variétés de Deligne-Lusztig. Les hypothèses ont été choisies pour les applications des parties à venir.

Fixons un élément $n$ de $\mathbf{G}$, un sous-groupe parabolique $\mathbf{P}$ de $\mathbf{G}$ de radical unipotent $\mathbf{V}$ et soit $\mathbf{L}$ un complément de Levi $n F$-stable $\left((n F)^{\delta}\right.$ est alors un endomorphisme de

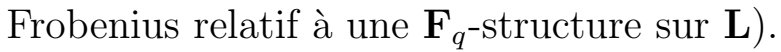

Fixons aussi un sous-groupe fermé connexe $n F$-stable $\mathbf{H}$ de $\mathbf{L}$, un sous-groupe distingué $\mathbf{H}^{\prime}$ de $\mathbf{H}$ (non nécessairement $n F$-stable) et posons

$$
\mathbf{K}=\left\{h \in \mathbf{H} \mid h^{-1} n F \quad h \in \mathbf{H}^{\prime}\right\} .
$$

Puisque $\mathbf{H}^{\prime}$ est distingué dans $\mathbf{H}, \mathbf{K}$ est un sous-groupe fermé de $\mathbf{H}$. D'autre part, $\mathbf{H}^{n F} \subset \mathbf{K}$.

Posons maintenant

$$
\mathbf{Y}=\left\{g \mathbf{V} \in \mathbf{G} / \mathbf{V} \mid g^{-1} F(g) \in \mathbf{H}^{\prime} \cdot\left(\mathbf{V} n^{F} \mathbf{V}\right)\right\}
$$

et

$$
\mathbf{X}=\left\{g \mathbf{H} \cdot \mathbf{V} \in \mathbf{G} /(\mathbf{H} \cdot \mathbf{V}) \mid g^{-1} F(g) \in \mathbf{H} \cdot\left(\mathbf{V}{ }^{F} \mathbf{V}\right)\right\}
$$


Alors, le groupe $\mathbf{G}^{F}$ agit par translation à gauche sur $\mathbf{Y}$ et $\mathbf{X}$, le groupe $\mathbf{K}$ agit librement par translation à droite sur $\mathbf{Y}$ et le morphisme

$$
\pi: \begin{aligned}
\mathbf{Y} & \longrightarrow \mathbf{X} \\
g \mathbf{V} & \longmapsto g \mathbf{H} \cdot \mathbf{V}
\end{aligned}
$$

est $\mathbf{G}^{F}$-équivariant. De plus, ses fibres sont des $\mathbf{K}$-orbites.

Proposition 5.1. Le morphisme $\pi$ induit un isomorphisme $\mathbf{Y} / \mathbf{K} \stackrel{\sim}{\rightarrow} \mathbf{X}$.

Démonstration. Tout d'abord,

$$
\mathbf{Y} / \mathbf{K}=\left\{g \mathbf{K} \cdot \mathbf{V} \in \mathbf{G} /(\mathbf{K} \cdot \mathbf{V}) \mid g^{-1} F(g) \in \mathbf{H}^{\prime} \cdot\left(\mathbf{V} n^{F} \mathbf{V}\right)\right\} .
$$

Notons

$$
\begin{aligned}
& \pi^{\prime}: \mathbf{Y} / \mathbf{K} \longrightarrow \mathbf{X} \\
& g \mathbf{K} \cdot \mathbf{V} \longmapsto g \mathbf{H} \cdot \mathbf{V} .
\end{aligned}
$$

Il s'agit de montrer que $\pi^{\prime}$ est un isomorphisme de variétés. Nous allons pour cela construire un inverse explicite.

L'application

$$
\begin{array}{ccc}
\mathbf{H} \times \mathbf{V} n{ }^{F} \mathbf{V} & \longrightarrow \mathbf{H} \cdot\left(\mathbf{V} n{ }^{F} \mathbf{V}\right) \\
(h, x) & \longmapsto & h x
\end{array}
$$

est un isomorphisme de variétés. Nous noterons $\lambda: \mathbf{H} \cdot\left(\mathbf{V} n{ }^{F} \mathbf{V}\right) \rightarrow \mathbf{H}$ le composé de l'inverse de cet isomorphisme avec la première projection.

D'autre part, l'application de Lang $\mathbf{H} \rightarrow \mathbf{H}, g \mapsto g^{n F} g^{-1}$ est un morphisme étale, donc elle induit un isomorphisme $\gamma_{0}: \mathbf{H} / \mathbf{H}^{n F} \stackrel{\sim}{\rightarrow} \mathbf{H}$. Nous noterons $\gamma$ le morphisme composé

$$
\mathbf{H} \stackrel{\gamma_{0}^{-1}}{\longrightarrow} \mathbf{H} / \mathbf{H}^{n F} \longrightarrow \mathbf{G} /(\mathbf{K} \cdot \mathbf{V}),
$$

où la deuxième flèche est l'application canonique.

Pour finir, posons

$$
\mathbf{X}^{\prime}=\left\{g \in \mathbf{G} \mid g^{-1} F(g) \in \mathbf{H} \cdot\left(\mathbf{V}{ }^{F} \mathbf{V}\right)\right\},
$$

et notons

$$
\begin{array}{rlc}
\mu: \mathbf{X}^{\prime} & \longrightarrow & \mathbf{Y} / \mathbf{K} \\
g & \longmapsto & g \gamma\left(\lambda\left(g^{-1} F(g)\right)\right) \mathbf{K} \cdot \mathbf{V} .
\end{array}
$$

Montrons que $\mu$ est bien défini. Prenons $g \in \mathbf{X}^{\prime}$ et posons $h=\lambda\left(g^{-1} F(g)\right)$. On a

$$
\left(g \gamma_{0}^{-1}(h)\right)^{-1 F}\left(g \gamma_{0}^{-1}(h)\right) \in \gamma_{0}^{-1}(h)^{-1} h^{n F} \gamma_{0}^{-1}(h) \mathbf{V} n^{F} \mathbf{V}=\mathbf{V} n^{F} \mathbf{V}
$$

ce qui montre que $\mu$ est bien défini - c'est de plus un morphisme de variétés. Pour $g \in \mathbf{X}^{\prime}$, $h \in \mathbf{H}$ et $v \in \mathbf{V}$, on a

$$
\gamma_{0}^{-1}\left(\lambda\left((g h v)^{-1} F(g h v)\right)\right)=h^{-1} \gamma_{0}^{-1}\left(\lambda\left(g^{-1} F(g)\right)\right) .
$$


On en déduit que $\mu(g h v)=\mu(g)$. Par passage au quotient, $\mu$ induit un morphisme de variétés $\mu^{\prime}: \mathbf{X}^{\prime} / \mathbf{H} \cdot \mathbf{V}=\mathbf{X} \rightarrow \mathbf{Y} / \mathbf{K}$. C'est l'isomorphisme inverse de $\pi^{\prime}$. Cela complète la preuve de la proposition.

5.2. Définition. Pour $w \in W$ (respectivement $\left.n \in N_{\mathbf{G}}(\mathbf{T})\right)$ et pour $g$ et $h$ deux éléments de $\mathbf{G}$, nous écrirons $g \mathbf{B} \stackrel{w}{w} h \mathbf{B}$ (respectivement $g \mathbf{U} \stackrel{n}{\rightarrow} h \mathbf{U}$ ) pour dire que $g^{-1} h \in \mathbf{B} w \mathbf{B}$ (respectivement $\left.g^{-1} h \in \mathbf{U} n \mathbf{U}\right)$. Pour $\mathbf{w}=\left(w_{1}, \ldots, w_{r}\right) \in \Sigma(W)$ et pour $\mathbf{n}=\left(n_{1}, \ldots, n_{r}\right) \in$ $\Sigma\left(N_{\mathbf{G}}(\mathbf{T})\right)$, nous posons

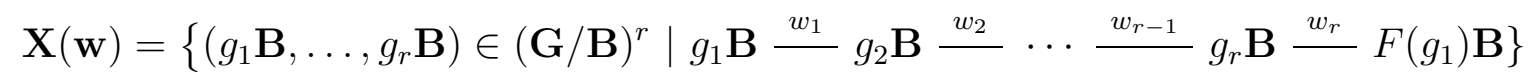

et

$$
\mathbf{Y}(\mathbf{n})=\left\{\left(g_{1} \mathbf{U}, \ldots, g_{r} \mathbf{U}\right) \in(\mathbf{G} / \mathbf{U})^{r} \mid g_{1} \mathbf{U} \stackrel{n_{1}}{g_{2}} \mathbf{U} \stackrel{n_{2}}{\cdots} \stackrel{n_{r-1}}{g_{r}} \mathbf{U} \stackrel{n_{r}}{F} F\left(g_{1}\right) \mathbf{U}\right\} .
$$

Supposons $\mathbf{w}=\overline{\mathbf{n}}$. Alors, le groupe $\mathbf{T}^{\mathbf{w} F}$ agit à droite sur $\mathbf{Y}(\mathbf{n})$ de la manière suivante : si $\left(g_{1} \mathbf{U}, \ldots, g_{r} \mathbf{U}\right) \in \mathbf{Y}(\mathbf{n})$ et si $t \in \mathbf{T}^{\mathbf{w} F}$, alors

$$
\left(g_{1} \mathbf{U}, \ldots, g_{r} \mathbf{U}\right) \cdot t=\left(g_{1} t \mathbf{U}, g_{2}{ }^{n_{1}^{-1}} t \mathbf{U}, \ldots, g_{r}{ }^{\left(n_{1} \ldots n_{r-1}\right)^{-1}} t \mathbf{U}\right)
$$

Notons que cette action est libre. D'autre part, $\mathbf{G}^{F}$ agit diagonalement sur $\mathbf{Y}(\mathbf{n})$ par multiplication à gauche et cette action commute avec celle de $\mathbf{T}^{\mathbf{w} F}$. Cela fait de $\mathbf{Y}(\mathbf{n})$ une $\mathbf{G}^{F}$-variété- $\mathbf{T}^{\mathbf{w} F}$.

Nous noterons $\mathbf{Y}^{\mathbf{G}}(\mathbf{n})$ ou $\mathbf{Y}^{\mathbf{G}, F}(\mathbf{n})$ (respectivement $\mathbf{X}^{\mathbf{G}}(\mathbf{w})$ ou $\mathbf{X}^{\mathbf{G}, F}(\mathbf{w})$ ) la variété $\mathbf{Y}(\mathbf{n})$ (respectivement $\mathbf{X}(\mathbf{w})$ ) lorsque nous aurons besoin de préciser le groupe ambiant, voire l'isogénie considérée.

Remarque 5.2. Posons $\mathbf{G}^{\prime}=\mathbf{G}^{r}, \mathbf{B}^{\prime}=\mathbf{B}^{r}, \mathbf{U}^{\prime}=\mathbf{U}^{r}, \mathbf{T}^{\prime}=\mathbf{T}^{r}, W^{\prime}=W^{r}$ et

et

$$
\begin{aligned}
& F^{\prime}: \quad \mathbf{G}^{\prime} \quad \longrightarrow \quad \mathbf{G}^{\prime} \\
& \left(g_{1}, \ldots, g_{r}\right) \longmapsto\left(g_{2}, \ldots, g_{r}, F\left(g_{1}\right)\right) .
\end{aligned}
$$

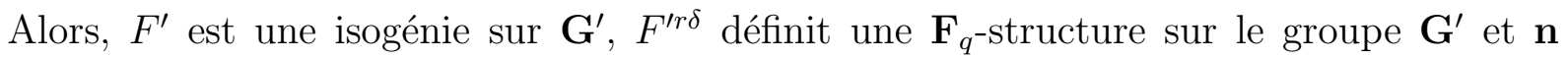
s'identifie à un élément du groupe $N_{\mathbf{G}^{\prime}}\left(\mathbf{T}^{\prime}\right)$. On vérifie que les applications

$$
\begin{aligned}
\mathbf{T}^{\mathbf{n} F} & \stackrel{\sim}{\rightarrow} \mathbf{T}^{\prime \mathbf{n} F^{\prime}} \\
t & \mapsto\left(t,,_{1}^{n^{-1}} t, \ldots,{ }^{\left(n_{1} \ldots n_{r-1}\right)^{-1}} t\right) \\
\mathbf{G}^{F} & \stackrel{\sim}{\rightarrow} \mathbf{G}^{\prime F^{\prime}} \\
g & \mapsto(g, \ldots, g)
\end{aligned}
$$

sont des isomorphismes de groupes et que l'application

$$
\begin{aligned}
& \mathbf{Y}^{\mathbf{G}, F}(\mathbf{n}) \quad \longrightarrow \quad \mathbf{Y}^{\mathbf{G}^{\prime}, F^{\prime}}(\mathbf{n}) \\
& \left(g_{1} \mathbf{U}, \ldots, g_{r} \mathbf{U}\right) \longmapsto\left(g_{1}, \ldots, g_{r}\right) \mathbf{U}^{\prime}
\end{aligned}
$$

est un isomorphisme de $\mathbf{G}^{F}$-variétés- $\mathbf{T}^{\mathbf{n} F}$, où $\mathbf{Y}^{\mathbf{G}^{\prime}, F^{\prime}}(\mathbf{n})$ est vue comme une $\mathbf{G}^{F}$-variété$\mathbf{T}^{\mathbf{n} F}$ via les isomorphismes précédents. 
Une interprétation analogue s'en déduit pour les variétés $\mathbf{X}^{\mathbf{G}, F}(\mathbf{w})$. Cela montre que les variétés de Deligne-Lusztig généralisées ne sont en fait que des variétés de Deligne-Lusztig ordinaires pour un groupe différent.

Le morphisme canonique

$$
\begin{aligned}
& \pi_{\mathbf{n}}: \quad \mathbf{Y}(\mathbf{n}) \quad \longrightarrow \quad \mathbf{X}(\mathbf{w}) \\
& \left(g_{1} \mathbf{U}, \ldots, g_{r} \mathbf{U}\right) \longmapsto\left(g_{1} \mathbf{B}, \ldots, g_{r} \mathbf{B}\right)
\end{aligned}
$$

induit un isomorphisme de $\mathbf{G}^{F}$-variétés

$$
\mathbf{Y}(\mathbf{n}) / \mathbf{T}^{\mathbf{w} F \stackrel{\sim}{\rightarrow}} \mathbf{X}(\mathbf{w}) .
$$

Pour voir cela, il faut se ramener au cas où $r=1$ en utilisant la remarque 5.2, puis appliquer la proposition 5.1 dans le cas où $\mathbf{P}=\mathbf{B}, \mathbf{L}=\mathbf{H}=\mathbf{T}, n=n_{1}$ et $\mathbf{H}^{\prime}=\{1\}$.

Puisque $\mathbf{Y}(\mathbf{n})$ est une $\mathbf{G}^{F}$-variété- $\mathbf{T}^{\mathbf{w} F}$, elle induit deux foncteurs entre catégories dérivées qui seront notés

et

$$
\begin{array}{cccc}
\mathcal{R}_{\mathbf{n}}: D^{b}\left(\Lambda \mathbf{T}^{\mathbf{w} F}\right) & \longrightarrow & D^{b}\left(\Lambda \mathbf{G}^{F}\right) \\
C & \longmapsto & \mathrm{R}_{c}(\mathbf{Y}(\mathbf{n})) \otimes_{\Lambda \mathbf{T}^{\mathbf{w} F}}^{\mathrm{L}} C \\
\mathcal{S}_{\mathbf{n}}: D^{b}\left(\Lambda \mathbf{T}^{\mathbf{w} F}\right) & \longrightarrow & D^{b}\left(\Lambda \mathbf{G}^{F}\right) \\
C & \longmapsto & \mathrm{R} \Gamma(\mathbf{Y}(\mathbf{n})) \otimes_{\Lambda \mathbf{T}^{\mathbf{w} F}}^{\mathrm{L}} C .
\end{array}
$$

Ce sont les foncteurs $\mathcal{R}_{\mathbf{T}^{\mathbf{w} F}}^{\mathbf{G}^{F}}(\mathbf{Y}(\mathbf{n}))$ et $\mathcal{S}_{\mathbf{T} \mathbf{w} F}^{\mathbf{G}^{F}}(\mathbf{Y}(\mathbf{n}))$ de $\S 3.2$.

Le foncteur $\mathcal{R}_{\mathbf{n}}$ induit une application linéaire entre les groupes de Grothendieck des catégories $D^{b}\left(\Lambda \mathbf{T}^{\mathbf{w} F}\right)$ et $D^{b}\left(\Lambda \mathbf{G}^{F}\right)$. Cette application sera notée

$$
\mathrm{R}_{\mathbf{n}}: \mathrm{K}\left(\Lambda \mathrm{T}^{\mathrm{w} F}\right) \longrightarrow \mathrm{K}\left(\Lambda \mathrm{G}^{F}\right)
$$

Les variétés $\mathbf{X}(\mathbf{w})$ et $\mathbf{Y}(\mathbf{n})$ ne dépendent, à isomorphisme près, que des images de $\mathbf{w}$ et $\mathbf{n}$ dans $B$ [DiMiRo] :

Proposition 5.4. Soient $\mathbf{v}$ et $\mathbf{w}$ deux suites d'éléments de $W$ telles que $\sigma(\mathbf{v})=\sigma(\mathbf{w})$. Alors, les $\mathbf{G}^{F}$-variétés $\mathbf{X}(\mathbf{w})$ et $\mathbf{X}(\mathbf{v})$ sont isomorphes. En particulier, une décomposition réduite de $\sigma(\mathbf{w})$ dans $B$ fournit une suite $\mathbf{v} \in \Sigma(S)$ telle que $\mathbf{X}(\mathbf{w})$ est isomorphe à $\mathbf{X}(\mathbf{v})$.

De même, soient $\mathbf{m}$ et $\mathbf{n}$ deux suites d'éléments de $N_{\mathbf{G}}(\mathbf{T})$ telles que $\dot{\sigma}(\mathbf{m}) \mathbf{T}=\dot{\sigma}(\mathbf{n}) \mathbf{T}$. Alors, on a un isomorphisme de $\mathbf{G}^{F}$-variétés- $\mathbf{T}{ }^{\mathbf{n} F}$ entre $\mathbf{Y}(\mathbf{m})$ et $\mathbf{Y}(\mathbf{n})$. Par conséquent, il existe $\mathbf{w} \in \Sigma(S)$ telle que $\mathbf{Y}(\mathbf{n})$ est isomorphe à $\mathbf{Y}(\dot{\mathbf{w}})$.

Démonstration. Soit $i$ tel que $l\left(\bar{n}_{i} \bar{n}_{i+1}\right)=l\left(\bar{n}_{i}\right)+l\left(\bar{n}_{i+1}\right)$. Alors, on a un isomorphisme de $\mathbf{G}^{F}$-variétés-T ${ }^{\mathbf{w}} F$

$$
\begin{aligned}
\mathbf{Y}(\mathbf{n}) & \stackrel{\sim}{\rightarrow} \mathbf{Y}\left(n_{1}, \ldots, n_{i-1}, n_{i} n_{i+1}, n_{i+2}, \ldots, n_{r}\right), \\
\left(g_{1} \mathbf{U}, \ldots, g_{r} \mathbf{U}\right) & \mapsto\left(g_{1} \mathbf{U}, \ldots, g_{i-1} \mathbf{U}, g_{i+1} \mathbf{U}, \ldots, g_{r} \mathbf{U}\right) .
\end{aligned}
$$

Ceci montre que $\mathbf{Y}(\mathbf{n})$ et $\mathbf{Y}(\mathbf{m})$ sont isomorphes lorsque $\dot{\sigma}(\mathbf{m})=\dot{\sigma}(\mathbf{n})$. 
Supposons maintenant $n_{r} \in \mathbf{T}$. Soit $t \in \mathbf{T}$ tel que $t^{-1} \mathbf{n} F t={ }^{\mathbf{n}} n_{r}$. Alors, on a un isomorphisme de $\mathbf{G}^{F}$-variétés- $\mathbf{T}^{\mathbf{w} F}$

$$
\begin{aligned}
\mathbf{Y}(\mathbf{n}) & \stackrel{\sim}{\rightarrow} \mathbf{Y}\left(n_{1}, \ldots, n_{r-1}\right), \\
\left(g_{1} \mathbf{U}, \ldots, g_{r} \mathbf{U}\right) & \mapsto\left(g_{1} t \mathbf{U}, g_{2}{ }^{n_{1}^{-1}} t \mathbf{U}, \ldots, g_{r-1} n_{r-2}^{-1} \cdots n_{1}^{-1} t \mathbf{U}\right) .
\end{aligned}
$$

On en déduit donc que $\mathbf{Y}(\mathbf{n})$ et $\mathbf{Y}(\mathbf{m})$ sont isomorphes lorsque $\dot{\sigma}(\mathbf{m}) \mathbf{T}=\dot{\sigma}(\mathbf{n}) \mathbf{T}$. Puisque $\mathbf{X}(\mathbf{w}) \simeq \mathbf{Y}(\mathbf{n}) / \mathbf{T}^{\mathbf{w} F}$, lorsque $\mathbf{w}$ est l'image de $\mathbf{n}$, on en déduit aussi la première partie de la proposition.

Remarque 5.6. D'après la proposition 5.4, l'étude des variétés $\mathbf{X}(\mathbf{w})$ et $\mathbf{Y}(\dot{\mathbf{w}})$ où $\mathbf{w} \in$ $\Sigma(W)$ se ramène au cas où $\mathbf{w} \in \Sigma(S)$.

Proposition 5.7. La variété $\mathbf{Y}(\mathbf{n})$ est quasi-affine, lisse et purement de dimension $l(\mathbf{n})$. Les stabilisateurs des points de $\mathbf{Y}(\mathbf{n})$ dans $\mathbf{G}^{F} \times \mathbf{T}^{\mathbf{n} F}$ sont des p-groupes.

Démonstration. La remarque 5.2 ramène la preuve de la proposition au cas de variétés de Deligne-Lusztig ordinaires. Alors, la quasi-affinité est due à Haastert [Ha, théorème 2.3] et les autres propriétés sont faciles [DeLu, §1.3].

Remarque 5.8. Il est connu que les variétés $\mathbf{Y}(\mathbf{n})$ sont affines pour $q^{1 / \delta}$ assez grand [DeLu, théorème 9.7], mais ce résultat demeure inconnu pour $q^{1 / \delta}$ quelconque.

Remarque 5.9. Pour $n \in N_{\mathbf{G}}(\mathbf{T})$, posons

$$
\mathbf{Y}^{*}(n)=\left\{g\left(\mathbf{U} \cap{ }^{n} \mathbf{U}\right) \in \mathbf{G} /\left(\mathbf{U} \cap{ }^{n} \mathbf{U}\right) \mid g^{-1} F(g) \in n \mathbf{U}\right\} .
$$

Alors, l'application canonique $\mathbf{Y}^{*}(n) \rightarrow \mathbf{Y}(n), g\left(\mathbf{U} \cap{ }^{n} \mathbf{U}\right) \mapsto g \mathbf{U}$ est un isomorphisme de $\mathbf{G}^{F}$-variétés- $\mathbf{T}^{n F}$. On trouve dans la littérature indifféremment l'un ou l'autre de ces modèles.

Remarque 5.10. Puisque la variété $\mathbf{X}(\mathbf{w})$ est lisse, la dualité de Poincaré fournit un isomorphisme fonctoriel de $\Lambda \mathbf{G}^{F}$-modules

$$
\left(\left(\mathcal{R}_{\mathbf{n}} M\right)^{*}\right)^{\mathrm{op}} \simeq \mathcal{S}_{\mathbf{n}}\left(\left(M^{*}\right)^{\mathrm{op}}\right)[-2 l(\mathbf{w})]
$$

pour tout tout $\Lambda \mathbf{T}^{\mathbf{w} F}$-module $M$, libre sur $\Lambda$.

5.3. Liens entre les groupes $\mathbf{G}, \tilde{\mathbf{G}}$ et $\hat{\mathbf{G}}$. En identifiant les groupes de Weyl de $\mathbf{G}, \tilde{\mathbf{G}}$ et $\hat{\mathbf{G}}$ relatifs à $\mathbf{T}, \tilde{\mathbf{T}}$ et $\hat{\mathbf{T}}$ respectivement, nous noterons, lorsque $\mathbf{w}$ est une suite d'éléments de $W, \tilde{\mathbf{X}}(\mathbf{w})$ et $\hat{\mathbf{X}}(\mathbf{w})$ les variétés définies grâce aux groupes $\tilde{\mathbf{G}}$ et $\hat{\mathbf{G}}$ respectivement. De même, si $\mathbf{n}$ est une suite d'éléments de $N_{\tilde{\mathbf{G}}}(\tilde{\mathbf{T}})$ ou de $N_{\hat{\mathbf{G}}}(\hat{\mathbf{T}})$, on définit des variétés $\tilde{\mathbf{Y}}(\mathbf{n})$ et $\hat{\mathbf{Y}}(\mathbf{n})$ et nous noterons $\tilde{\mathcal{R}}_{\mathbf{n}}$ et $\hat{\mathcal{R}}_{\mathbf{n}}$ les foncteurs associés aux variétés $\tilde{\mathbf{Y}}(\mathbf{n})$ et $\hat{\mathbf{Y}}(\mathbf{n})$ respectivement.

Dans ce paragraphe, nous allons rappeler les liens entre ces variétés. Dans le cas des variétés $\mathbf{X}(\mathbf{w}), \tilde{\mathbf{X}}(\mathbf{w})$ et $\hat{\mathbf{X}}(\mathbf{w})$, la comparaison est facile. Les morphismes canoniques $\mathbf{G} \rightarrow \tilde{\mathbf{G}}$ et $\hat{\mathbf{G}} \rightarrow \mathbf{G}$ induisent des isomorphismes $\mathbf{G} / \mathbf{B} \stackrel{\sim}{\rightarrow} \tilde{\mathbf{G}} / \tilde{\mathbf{B}}$ et $\hat{\mathbf{G}} / \hat{\mathbf{B}} \stackrel{\sim}{\rightarrow} \mathbf{G} / \mathbf{B}$, d'où

Proposition 5.12. Soit $\mathbf{w}$ une suite d'éléments de $W$. Alors, les $\mathbf{G}^{F}$-variétés $\mathbf{X}(\mathbf{w})$, $\tilde{\mathbf{X}}(\mathbf{w})$ et $\hat{\mathbf{X}}(\mathbf{w})$ sont canoniquement isomorphes. 
Les relations entre les variétés $\mathbf{Y}(\mathbf{n}), \tilde{\mathbf{Y}}(\mathbf{n})$ et $\hat{\mathbf{Y}}(\mathbf{n})$, sont un peu plus compliquées. Commençons par les variétés $\tilde{\mathbf{Y}}(\mathbf{n})$.

Le produit fournit des isomorphismes canoniques $\tilde{\mathbf{G}}^{F} \times_{\mathbf{G}^{F}} \mathbf{G}^{F} \stackrel{\sim}{\rightarrow} \tilde{\mathbf{G}}^{F}$ et $\mathbf{G}^{F} \times{ }_{\mathbf{T}^{\mathbf{w}} F}$ $\tilde{\mathbf{T}}^{\mathbf{w} F} \stackrel{\sim}{\rightarrow} \tilde{\mathbf{G}}^{F}$. On en déduit :

Proposition 5.13. Soit $\mathbf{n}$ une suite d'éléments de $N_{\mathbf{G}}(\mathbf{T})$. Alors on a des isomorphismes canoniques

$$
\tilde{\mathbf{Y}}(\mathbf{n}) \stackrel{\sim}{\rightarrow} \tilde{\mathbf{G}}^{F} \times_{\mathbf{G}^{F}} \mathbf{Y}(\mathbf{n}) \stackrel{\sim}{\rightarrow} \mathbf{Y}(\mathbf{n}) \times_{\mathbf{T}^{\mathbf{w} F}} \tilde{\mathbf{T}}^{\mathbf{w} F} .
$$

Démonstration. Grâce à la remarque 5.2, on peut supposer que $\mathbf{n}=\left(n_{1}\right)$, où $n_{1} \in N_{\mathbf{G}}(\mathbf{T})$. Dans ce cas, la preuve est par exemple analogue à [Bo, lemme 2.1.2 (b)].

Soit $\mathbf{n}$ une suite d'éléments de $N_{\mathbf{G}}(\mathbf{T})$. La proposition 5.13 a des conséquences au niveau des foncteurs $\mathcal{R}_{\mathbf{n}}$ et $\tilde{\mathcal{R}}_{\mathbf{n}}$. En effet, on en déduit que

$$
\mathrm{R} \Gamma_{c}(\tilde{\mathbf{Y}}(\mathbf{n})) \simeq \Lambda \tilde{\mathbf{G}}^{F} \otimes_{\Lambda \mathbf{G}^{F}} \mathrm{R} \Gamma_{c}(\mathbf{Y}(\mathbf{n})) \quad \text { dans } D^{b}\left(\Lambda \tilde{\mathbf{G}}^{F} \otimes \Lambda \mathbf{T}^{\mathbf{w} F}\right)
$$

et que

$$
\mathrm{R} \Gamma_{c}(\tilde{\mathbf{Y}}(\mathbf{n})) \simeq \mathrm{R} \Gamma_{c}(\mathbf{Y}(\mathbf{n})) \otimes_{\Lambda \mathbf{T} w F} \Lambda \tilde{\mathbf{T}}^{\mathbf{w} F} \quad \text { dans } D^{b}\left(\Lambda \mathbf{G}^{F} \otimes \Lambda \tilde{\mathbf{T}}^{\mathbf{w} F}\right)
$$

Par suite, on a

$$
\tilde{\mathcal{R}}_{\mathbf{n}} \circ \operatorname{Ind}_{\mathbf{T}^{\mathbf{w} F}}^{\tilde{\mathbf{T}}^{\mathbf{w} F}} \simeq \operatorname{Ind}_{\mathbf{G}^{F}}^{\tilde{\mathbf{G}}^{F}} \circ \mathcal{R}_{\mathbf{n}}
$$

et

$$
\mathcal{R}_{\mathbf{n}} \circ \operatorname{Res}_{\mathbf{T}^{\mathbf{w} F}}^{\tilde{\mathbf{T}}^{\mathrm{w} F}} \simeq \operatorname{Res}_{\mathbf{G}^{F}}^{\tilde{\mathbf{G}}^{F}} \circ \tilde{\mathcal{R}}_{\mathbf{n}} .
$$

Remarque 5.16. Les isomorphismes (5.14) et (5.15) induisent les égalités

$$
\tilde{\mathrm{R}}_{\mathbf{n}} \circ \operatorname{Ind}_{\mathbf{T}^{\mathbf{w} F}}^{\tilde{\mathbf{T}}^{\mathbf{w} F}}=\operatorname{Ind}_{\mathbf{G}^{F}}^{\tilde{\mathbf{G}}^{F}} \circ \mathbf{R}_{\mathbf{n}}
$$

et

$$
\mathrm{R}_{\mathbf{n}} \circ \operatorname{Res}_{\mathbf{T}^{\mathbf{w} F}}^{\tilde{\mathbf{T}}^{\mathbf{w} F}}=\operatorname{Res}_{\mathbf{G}^{F}}^{\tilde{\mathbf{G}}^{F}} \circ \tilde{\mathrm{R}}_{\mathbf{n}} .
$$

Concernant les rapports entre les variétés $\hat{\mathbf{Y}}(\hat{\mathbf{n}})$ et $\mathbf{Y}(\mathbf{n})$, nous avons le résultat suivant (rappelons que $\rho: \hat{\mathbf{G}} \rightarrow \mathbf{G}$ désigne la projection canonique) :

Proposition 5.19. Soit $\hat{\mathbf{n}}=\left(\hat{n}_{1}, \ldots, \hat{n}_{r}\right)$ une suite finie d'éléments de $N_{\hat{\mathbf{G}}}(\hat{\mathbf{T}})$ et posons $\mathbf{n}=\left(\rho\left(\hat{n}_{1}\right), \ldots, \rho\left(\hat{n}_{r}\right)\right)$. Alors, l'application $\rho$ induit des isomorphismes de $\mathbf{G}^{F}$-variétés$\mathbf{T}^{\mathbf{w} F}$

$$
\hat{\mathbf{Y}}(\hat{\mathbf{n}}) / \mathbf{C}^{F} \stackrel{\sim}{\rightarrow} \mathbf{Y}(\mathbf{n}) \quad \text { et } \quad \mathbf{C}^{F} \backslash \hat{\mathbf{Y}}(\hat{\mathbf{n}}) \stackrel{\sim}{\rightarrow} \mathbf{Y}(\mathbf{n}) .
$$

Démonstration. Encore une fois, comme pour la proposition 5.13, on peut supposer que $\mathbf{n}$ est une suite formée d'un seul élément. Dans ce cas, il suffit d'appliquer la proposition 5.1, en remplaçant le groupe $\mathbf{G}$ par $\hat{\mathbf{G}}, \mathbf{P}$ par $\hat{\mathbf{B}}, \mathbf{L}$ par $\hat{\mathbf{T}}, n$ par $\hat{n}_{1}$, le groupe $\mathbf{H}$ par $\mathbf{C}$, et le groupe $\mathbf{H}^{\prime}$ par $\{1\}$. Dans ce cas, la variété qui était notée $\mathbf{Y}$ devient la variété $\hat{\mathbf{Y}}(\hat{\mathbf{n}})$, tandis que $\mathbf{X}$ devient $\mathbf{Y}(\mathbf{n})$ et $\mathbf{K}=\mathbf{C}^{F}$. Cela complète la preuve de la proposition 5.19 . 
Si $\operatorname{Res}_{\hat{\mathbf{G}}^{F}}^{\mathbf{G}^{F}}: D^{b}\left(\Lambda \mathbf{G}^{F}\right) \longrightarrow D^{b}\left(\Lambda \hat{\mathbf{G}}^{F}\right)$ désigne le foncteur de restriction à travers $\rho$, alors la proposition 5.19 montre que

$$
\hat{\mathcal{R}}_{\hat{\mathbf{n}}} \circ \operatorname{Res}_{\hat{\mathbf{T}}^{\mathbf{w} F}}^{\mathbf{T}^{\mathbf{w} F}} \simeq \operatorname{Res}_{\hat{\mathbf{G}}^{F}}^{\mathbf{G}^{F}} \circ \mathcal{R}_{\mathbf{n}} .
$$

Remarque 5.21. Tous les résultats énoncés dans ce $\S 5.3$ restent vrais même lorsque le centre de $\tilde{\mathbf{G}}$ n'est pas connexe ou lorsque les coracines de $\hat{\mathbf{G}}$ ne sont pas injectives. D'autre part, si $\breve{\mathbf{G}}$ est un groupe algébrique muni d'une isogénie $F: \check{\mathbf{G}} \rightarrow \check{\mathbf{G}}$ telle que $F^{\delta}$ soit l'endomorphisme de Frobenius associé à une $\mathbf{F}_{q}$-structure sur $\check{\mathbf{G}}$, et si $\gamma: \check{\mathbf{G}} \rightarrow \mathbf{G}$ est un morphisme de groupes algébriques commutant avec $F$ et induisant un isomorphisme de groupes, alors les groupes finis $\check{\mathbf{G}}^{F}$ et $\mathbf{G}^{F}$ sont isomorphes via $\gamma$ et $\gamma$ induit des équivalences équivariantes de sites étales entre les variétés $\mathbf{Y}^{\check{\mathbf{G}}}(\check{\mathbf{n}})$ et $\mathbf{Y}^{\mathbf{G}}(\mathbf{n})$ (avec des notations évidentes). En particulier, on a $\mathcal{R}_{\check{\mathbf{n}}}^{\check{\mathbf{G}}} \circ \operatorname{Res}_{\check{\mathbf{T}}^{\check{\mathbf{n}} F}}^{\mathbf{T} F}=\operatorname{Res}_{\check{\mathbf{G}}^{F}}^{\mathbf{G}^{F}} \circ \mathcal{R}_{\mathbf{n}}^{\mathbf{G}}$.

\section{ReCOllements}

6.1. Construction. Dans ce paragraphe 6.1, et dans ce paragraphe seulement, nous supposerons que $\mathbf{G}=\hat{\mathbf{G}}$. Soient $\mathbf{v}$ et $\mathbf{w}$ deux suites d'éléments de $\bar{S}$ telles que $\mathbf{v} \leq \mathbf{w}$. Nous noterons $\mathbf{w}=\left(s_{1}, \ldots, s_{r}\right)$ et $\mathbf{v}=\left(s_{1}^{\prime}, \ldots, s_{r}^{\prime}\right)$. Posons

$$
\mathbf{X}(\mathbf{w} ; \mathbf{v})=\coprod_{\mathbf{v} \leq \mathbf{y} \leq \mathbf{w}} \mathbf{X}(\mathbf{y})
$$

Puisque $\mathbf{B} s \mathbf{B} \coprod \mathbf{B}$ est une sous-variété fermée lisse de $\mathbf{G}$ pour tout $s \in S$ (c'est même un sous-groupe parabolique de $\mathbf{G}), \mathbf{X}(\mathbf{w} ; \mathbf{v})$ est une sous-variété localement fermée lisse de $(\mathbf{G} / \mathbf{B})^{r}$. Nous allons ici construire un revêtement étale (en général non trivial) de la variété $\mathbf{X}(\mathbf{w} ; \mathbf{v})$ en recollant des quotients des variétés $\mathbf{Y}(\dot{\mathbf{y}})$ pour $\mathbf{v} \leq \mathbf{y} \leq \mathbf{w}$.

Soit $\alpha \in \Delta$. Alors, $\mathbf{U} \dot{s}_{\alpha} \mathbf{U}=\mathbf{U} \dot{s}_{\alpha} \mathbf{U}_{\alpha}\left(\mathbf{U} \cap{ }^{s_{\alpha}} \mathbf{U}\right)$. Cela montre que

$$
\mathbf{U} \dot{s}_{\alpha} \mathbf{U} \mathbf{T}_{\alpha^{\vee}} \coprod \mathbf{U} \mathbf{T}_{\alpha^{\vee}}=\mathbf{G}_{\alpha}\left(\mathbf{U} \cap{ }^{s_{\alpha}} \mathbf{U}\right)=\mathbf{G}_{\alpha} \mathbf{U} .
$$

En particulier, c'est une sous-variété lisse localement fermée de $\mathbf{G}$. Pour $1 \leq i \leq r$, on pose

$$
\mathcal{U}_{\mathbf{w}, \mathbf{v}}^{(i)}= \begin{cases}\mathbf{G}_{\alpha_{\mathbf{w}, i}} \mathbf{U} & \text { si } i \in I_{\mathbf{w}, \mathbf{v}} \\ \mathbf{U} \dot{s}_{i} \mathbf{U} & \text { sinon }\end{cases}
$$

où $I_{\mathbf{w}, \mathbf{v}}$ a été défini au $§ 4.4 .2$.

On définit alors la variété

$$
\begin{array}{r}
\mathbf{Y}^{\prime}(\mathbf{w} ; \mathbf{v})=\left\{\left(g_{1} \mathbf{U}, \ldots, g_{r} \mathbf{U}\right) \in(\mathbf{G} / \mathbf{U})^{r} \mid g_{i}^{-1} g_{i+1} \in \mathcal{U}_{\mathbf{w}, \mathbf{v}}^{(i)} \text { pour } 1 \leq i \leq r-1\right. \\
\text { et } \left.g_{r}^{-1} F\left(g_{1}\right) \in \mathcal{U}_{\mathbf{w}, \mathbf{v}}^{(r)}\right\} .
\end{array}
$$

C'est une sous-variété localement fermée lisse de $(\mathbf{G} / \mathbf{U})^{r}$, sur laquelle le groupe $\mathbf{G}^{F}$ agit diagonalement par multiplication à gauche. De plus, nous avons un morphisme canonique de $\mathbf{G}^{F}$-variétés $\pi_{\mathbf{w}, \mathbf{v}}^{\prime}: \mathbf{Y}^{\prime}(\mathbf{w} ; \mathbf{v}) \rightarrow \mathbf{X}(\mathbf{w} ; \mathbf{v})$ induit par la projection $(\mathbf{G} / \mathbf{U})^{r} \rightarrow(\mathbf{G} / \mathbf{B})^{r}$. 
D'autre part, pour $\left(g_{1} \mathbf{U}, \ldots, g_{r} \mathbf{U}\right) \in \mathbf{Y}^{\prime}(\mathbf{w} ; \mathbf{v})$ et pour $\left(t_{1}, \ldots, t_{r}\right) \in \mathbf{T}^{r}$, alors $\left(g_{1} t_{1} \mathbf{U}, \ldots, g_{r} t_{r} \mathbf{U}\right) \in \mathbf{Y}^{\prime}(\mathbf{w} ; \mathbf{v})$ si et seulement si $\left(t_{1}, \ldots, t_{r}\right) \in \mathbf{S}_{\mathbf{w}, \mathbf{v}}$.

Lemme 6.1. L'application canonique $\mathbf{Y}^{\prime}(\mathbf{w} ; \mathbf{v}) / \mathbf{S}_{\mathbf{w}, \mathbf{v}} \rightarrow \mathbf{X}(\mathbf{w} ; \mathbf{v})$ induite par $\pi_{\mathbf{w}, \mathbf{v}}^{\prime}$ est un isomorphisme de $\mathbf{G}^{F}$-variétés.

Démonstration. Puisque le morphisme est bijectif et que $\mathbf{X}(\mathbf{w} ; \mathbf{v})$ est lisse, il suffit de vérifier que c'est un isomorphisme au-dessus de l'ouvert $\mathbf{X}(\mathbf{w})$. Cela résulte alors de la remarque 5.2 et de la proposition 5.1 appliquée au groupe $\mathbf{G}^{\prime}=\mathbf{G}^{r}$ muni de l'isogénie définie dans la remarque 5.2 , avec $\mathbf{P}=\mathbf{B}^{r}, \mathbf{L}=\mathbf{H}=\mathbf{T}^{r}, n=\dot{\mathbf{w}}$, et $\mathbf{H}^{\prime}=\mathbf{T}_{\mathbf{w}, \mathbf{v}}^{(1)} \times \cdots \mathbf{T}_{\mathbf{w}, \mathbf{v}}^{(r)}$. Il faut noter qu'alors $\mathbf{K}=\mathbf{S}_{\mathbf{w}, \mathbf{v}}$.

Définissons

$$
\mathbf{Y}(\mathbf{w} ; \mathbf{v})=\mathbf{Y}^{\prime}(\mathbf{w} ; \mathbf{v}) / \mathbf{S}_{\mathbf{w}, \mathbf{v}}^{\circ}
$$

et notons $\pi_{\mathbf{w}, \mathbf{v}}: \mathbf{Y}(\mathbf{w}, \mathbf{v}) \rightarrow \mathbf{X}(\mathbf{w}, \mathbf{v})$ le morphisme de $\mathbf{G}^{F}$-variétés induit par $\pi_{\mathbf{w}, \mathbf{v}}^{\prime}$. Le lemme 6.1 montre que $\pi_{\mathbf{w}, \mathbf{v}}$ est un $\left(\mathbf{S}_{\mathbf{w}, \mathbf{v}} / \mathbf{S}_{\mathbf{w}, \mathbf{v}}^{\circ}\right)$-torseur.

Considérons maintenant une suite $\mathbf{y}$ telle que $\mathbf{v} \leq \mathbf{y} \leq \mathbf{w}$. Alors, l'injection canonique $\mathbf{Y}(\dot{\mathbf{y}}) \hookrightarrow \mathbf{Y}^{\prime}(\mathbf{w} ; \mathbf{v})$ est un morphisme de $\mathbf{G}^{F}$-variétés- $\mathbf{T}^{\mathbf{y} F}$ : ici, $\mathbf{Y}(\mathbf{w} ; \mathbf{v})$ est vue comme une variété- $\mathbf{T}^{\mathbf{y} F}$ via le morphisme $\mu_{\mathbf{y}}: \mathbf{T}^{\mathbf{y} F} \hookrightarrow \mathbf{S}_{\mathbf{w}, \mathbf{v}}$ défini dans le $\S 4.4 .3$. Puisque

$$
\mu_{\mathbf{y}}\left(\mathbf{T}^{\mathbf{y} F}\right) \cap \mathbf{S}_{\mathbf{w}, \mathbf{v}}^{\circ}=\mu_{\mathbf{y}}\left(N_{\mathbf{y}}\left(Y_{\mathbf{w}, \mathbf{v}}\right)\right),
$$

(cf proposition $4.11(4)$ ), cela induit un morphisme de $\mathbf{G}^{F}$-variétés- $\left(\mathbf{T}^{\mathbf{y} F} / N_{\mathbf{y}}\left(Y_{\mathbf{w}, \mathbf{v}}\right)\right.$ )

$$
\mathbf{Y}(\dot{\mathbf{y}}) / N_{\mathbf{y}}\left(Y_{\mathbf{w}, \mathbf{v}}\right) \longrightarrow \mathbf{Y}(\mathbf{w} ; \mathbf{v})
$$

Lemme 6.3. L'image du morphisme $\mathbf{Y}(\dot{\mathbf{y}}) \rightarrow \mathbf{Y}(\mathbf{w} ; \mathbf{v})$ est égale à $\pi_{\mathbf{w}, \mathbf{v}}^{-1}(\mathbf{X}(\mathbf{y}))$. Ce morphisme induit un isomorphisme de $\mathbf{G}^{F}$-variétés- $\left(\mathbf{T}^{\mathbf{y} F} / N_{\mathbf{y}}\left(Y_{\mathbf{w}, \mathbf{v}}\right)\right)$

$$
\mathbf{Y}(\dot{\mathbf{y}}) / N_{\mathbf{y}}\left(Y_{\mathbf{w}, \mathbf{v}}\right) \stackrel{\sim}{\rightarrow} \pi_{\mathbf{w}, \mathbf{v}}^{-1}(\mathbf{X}(\mathbf{y}))
$$

Démonstration. L'application $\mathbf{Y}(\dot{\mathbf{y}}) \rightarrow \mathbf{X}(\mathbf{y})$ est surjective. Donc, d'après le lemme 6.1, l'application

$$
\begin{array}{cccc}
\mathbf{Y}(\dot{\mathbf{y}}) \times_{\mu_{\mathbf{y}}\left(\mathbf{T}^{\mathbf{y} F}\right)} \mathbf{S}_{\mathbf{w}, \mathbf{v}} & \stackrel{\sim}{\rightarrow} & \pi_{\mathbf{w}, \mathbf{v}}^{\prime-1}(\mathbf{X}(\mathbf{y})) \\
(\gamma, \tau) & \mapsto & \gamma \cdot \tau
\end{array}
$$

est un isomorphisme de variétés. Le résultat découle alors de ce que

$$
\mathbf{S}_{\mathbf{w}, \mathbf{v}}=\mu_{\mathbf{y}}\left(\mathbf{T}^{\mathbf{y} F}\right) \cdot \mathbf{S}_{\mathbf{w}, \mathbf{v}}^{\circ},
$$

ce qui est démontré dans la proposition $4.11(2)$.

6.2. Le cas général. Revenons maintenant au cas général : nous ne supposons plus que $\mathbf{G}=\hat{\mathbf{G}}$. Le lecteur aura remarqué que le lemme 6.3 n'est plus nécessairement vrai (nous avons utilisé la proposition 4.11 (4), qui n'est pas vraie en général). C'est pourquoi nous allons devoir passer par le groupe $\hat{\mathbf{G}}$ pour définir un bon revêtement de la variété $\mathbf{X}(\mathbf{w} ; \mathbf{v})$. 
Notons $\hat{\mathbf{Y}}^{\prime}(\mathbf{w} ; \mathbf{v})$ (respectivement $\hat{\mathbf{S}}_{\mathbf{w}, \mathbf{v}}$ ) la variété (respectivement le groupe diagonalisable) définie (respectivement défini) dans le groupe $\mathbf{G}$ comme l'a été $\mathbf{Y}^{\prime}(\mathbf{w} ; \mathbf{v})$ (respectivement $\mathbf{S}_{\mathbf{w}, \mathbf{v}}$ ) dans le paragraphe précédent. Nous posons alors :

$$
\mathbf{Y}(\mathbf{w} ; \mathbf{v})=\hat{\mathbf{Y}}^{\prime}(\mathbf{w} ; \mathbf{v}) /\left(\hat{\mathbf{S}}_{\mathbf{w}, \mathbf{v}}^{\circ} \cdot \mathbf{C}^{F}\right),
$$

le groupe $\mathbf{C}^{F}$ agissant diagonalement $\operatorname{sur}(\hat{\mathbf{G}} / \hat{\mathbf{B}})^{r}$ par multiplication. Notons $\pi_{\mathbf{w}, \mathbf{v}}$ : $\mathbf{Y}(\mathbf{w} ; \mathbf{v}) \rightarrow \mathbf{X}(\mathbf{w} ; \mathbf{v})$ le morphisme canonique : c'est un $\left(\hat{\mathbf{S}}_{\mathbf{w}, \mathbf{v}} /\left(\hat{\mathbf{S}}_{\mathbf{w}, \mathbf{v}}^{\circ} \cdot \mathbf{C}^{F}\right)\right)$-torseur.

Compte tenu des propositions 5.12 et 5.19 et du lemme 6.3 , nous déduisons le résultat suivant.

Théorème 6.5. Le morphisme de $\mathbf{G}^{F}$-variétés

$$
\pi_{\mathbf{w}, \mathbf{v}}: \mathbf{Y}(\mathbf{w} ; \mathbf{v}) \rightarrow \mathbf{X}(\mathbf{w} ; \mathbf{v})
$$

est un $\mathbf{T}^{\mathbf{w} F} / N_{\mathbf{w}}\left(Y_{\mathbf{w}, \mathbf{v}}\right)$-torseur étale. De plus, pour $\mathbf{v} \leq \mathbf{y} \leq \mathbf{w}$, l'application canonique $\mathbf{Y}(\dot{\mathbf{y}}) \rightarrow \mathbf{Y}(\mathbf{w} ; \mathbf{v})$ induit un isomorphisme $\mathbf{Y}(\dot{\mathbf{y}}) / N_{\mathbf{y}}\left(Y_{\mathbf{w}, \mathbf{v}}\right) \stackrel{\sim}{\rightarrow} \pi_{\mathbf{w}, \mathbf{v}}^{-1}(\mathbf{X}(\mathbf{y}))$ de $\mathbf{G}^{F}$-variétés$\left(\mathbf{T}^{\mathbf{y} F} / N_{\mathbf{y}}\left(Y_{\mathbf{w}, \mathbf{v}}\right)\right)$ rendant commutatif le diagramme suivant

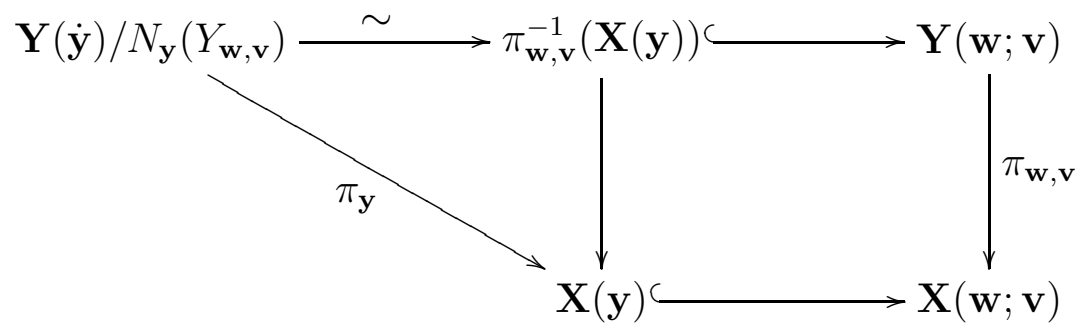

Remarque 6.7. Dans le diagramme 6.6, le revêtement étale $\mathbf{Y}(\dot{\mathbf{y}}) / N_{\mathbf{x}}\left(Y_{\mathbf{w}, \mathbf{v}}\right) \rightarrow \mathbf{X}(\mathbf{y})$ est un $\mathbf{T}^{\mathbf{y} F} / N_{\mathbf{y}}\left(Y_{\mathbf{w}, \mathbf{v}}\right)$-torseur, alors que le revêtement étale $\pi_{\mathbf{w}, \mathbf{v}}: \pi_{\mathbf{w}, \mathbf{v}}^{-1}(\mathbf{X}(\mathbf{y})) \rightarrow \mathbf{X}(\mathbf{y})$ est quant à lui un $\mathbf{T}^{\mathbf{w} F} / N_{\mathbf{w}}\left(Y_{\mathbf{w}, \mathbf{v}}\right)$-torseur. Ces descriptions sont compatibles, via l'isomorphisme canonique $\mathbf{T}^{\mathbf{y} F} / N_{\mathbf{y}}\left(Y_{\mathbf{w}, \mathbf{v}}\right) \stackrel{\sim}{\rightarrow} \mathbf{T}^{\mathbf{w} F} / N_{\mathbf{w}}\left(Y_{\mathbf{w}, \mathbf{v}}\right)$ (cf proposition 4.11 ).

6.3. Recollement de recollements. Soient $\mathbf{v} \leq \mathbf{x} \leq \mathbf{y} \leq \mathbf{w}$. La variété $\mathbf{X}(\mathbf{y} ; \mathbf{x})$ est une sous-variété localement fermée de $\mathbf{X}(\mathbf{w} ; \mathbf{v})$ et $\pi_{\mathbf{w}, \mathbf{v}}^{-1}(\mathbf{X}(\mathbf{y} ; \mathbf{x})) \rightarrow \mathbf{X}(\mathbf{y} ; \mathbf{x})$ est un $\mathbf{T}^{\mathbf{w} F} / N_{\mathbf{w}}\left(Y_{\mathbf{w}, \mathbf{v}}\right)$-torseur étale.

D'autre part, $\pi_{\mathbf{y}, \mathbf{x}}: \mathbf{Y}(\mathbf{y} ; \mathbf{x}) \rightarrow \mathbf{X}(\mathbf{y} ; \mathbf{x})$ est un $\mathbf{T}^{\mathbf{y} F} / N_{\mathbf{y}}\left(Y_{\mathbf{y}, \mathbf{x}}\right)$-torseur étale. D'après le corollaire 4.5 , on a $Y_{\mathbf{y}, \mathbf{x}} \subset Y_{\mathbf{w}, \mathbf{v}}$. Par conséquent, $\mathbf{T}^{\mathbf{y} F} / N_{\mathbf{y}}\left(Y_{\mathbf{w}, \mathbf{v}}\right)$ est un quotient de $\mathbf{T}^{\mathbf{y} F} / N_{\mathbf{y}}\left(Y_{\mathbf{y}, \mathbf{x}}\right)$ et le morphisme canonique $\mathbf{Y}(\mathbf{y} ; \mathbf{x}) /\left(N_{\mathbf{y}}\left(Y_{\mathbf{w}, \mathbf{v}}\right) / N_{\mathbf{y}}\left(Y_{\mathbf{y}, \mathbf{x}}\right)\right) \rightarrow \mathbf{X}(\mathbf{y} ; \mathbf{x})$ est un $\mathbf{T}^{\mathbf{y} F} / N_{\mathbf{y}}\left(Y_{\mathbf{w}, \mathbf{v}}\right)$-torseur étale. Mais d'après la proposition 4.4 (4), on a un isomorphisme canonique

$$
\mathbf{T}^{\mathbf{y} F} / N_{\mathbf{y}}\left(Y_{\mathbf{w}, \mathbf{v}}\right) \simeq \mathbf{T}^{\mathbf{w} F} / N_{\mathbf{w}}\left(Y_{\mathbf{w}, \mathbf{v}}\right) .
$$

Par conséquent, nous avons construit deux $\mathbf{T}^{\mathbf{w} F} / N_{\mathbf{w}}\left(Y_{\mathbf{w}, \mathbf{v}}\right)$-torseurs étales au-dessus de $\mathbf{X}(\mathbf{y} ; \mathbf{x})$. Le corollaire suivant montre qu'ils sont en fait isomorphes.

Corollaire 6.8. Le morphisme canonique $\mathbf{Y}(\mathbf{y} ; \mathbf{x}) \rightarrow \mathbf{Y}(\mathbf{w} ; \mathbf{v})$ induit un isomorphisme $\mathbf{G}^{F}$-équivariant de $\mathbf{T}^{\mathbf{w} F} / N_{\mathbf{w}}\left(Y_{\mathbf{w}, \mathbf{v}}\right)$-torseurs étales

$$
\mathbf{Y}(\mathbf{y} ; \mathbf{x}) /\left(N_{\mathbf{y}}\left(Y_{\mathbf{w}, \mathbf{v}}\right) / N_{\mathbf{y}}\left(Y_{\mathbf{y}, \mathbf{x}}\right)\right) \stackrel{\sim}{\rightarrow} \pi_{\mathbf{w}, \mathbf{v}}^{-1}(\mathbf{X}(\mathbf{y} ; \mathbf{x}))
$$


au-dessus de $\mathbf{X}(\mathbf{y} ; \mathbf{x})$.

Démonstration. Le morphisme canonique provient de l'inclusion $\mathbf{Y}^{\prime}(\mathbf{y} ; \mathbf{x}) \hookrightarrow \mathbf{Y}^{\prime}(\mathbf{w} ; \mathbf{v})$. Il est clair que son image est égale à la variété lisse $\pi_{\mathbf{w}, \mathbf{v}}^{-1}(\mathbf{X}(\mathbf{y} ; \mathbf{x}))$. Pour démontrer le corollaire 6.8, il suffit de démontrer que le morphisme

$$
\mathbf{Y}(\mathbf{y} ; \mathbf{x}) /\left(N_{\mathbf{y}}\left(Y_{\mathbf{w}, \mathbf{v}}\right) / N_{\mathbf{y}}\left(Y_{\mathbf{y}, \mathbf{x}}\right)\right) \longrightarrow \pi_{\mathbf{w}, \mathbf{v}}^{-1}(\mathbf{X}(\mathbf{y} ; \mathbf{x}))
$$

ainsi défini induit un isomorphisme au-dessus d'un ouvert de $\mathbf{X}(\mathbf{y} ; \mathbf{x})$. On peut par exemple prendre l'ouvert $\mathbf{X}(\mathbf{y})$ de $\mathbf{X}(\mathbf{y} ; \mathbf{x})$. Mais ce fait résulte de la commutativité du diagramme du théorème 6.5.

Nous résumons le corollaire 6.8 dans le diagramme commutatif suivant :

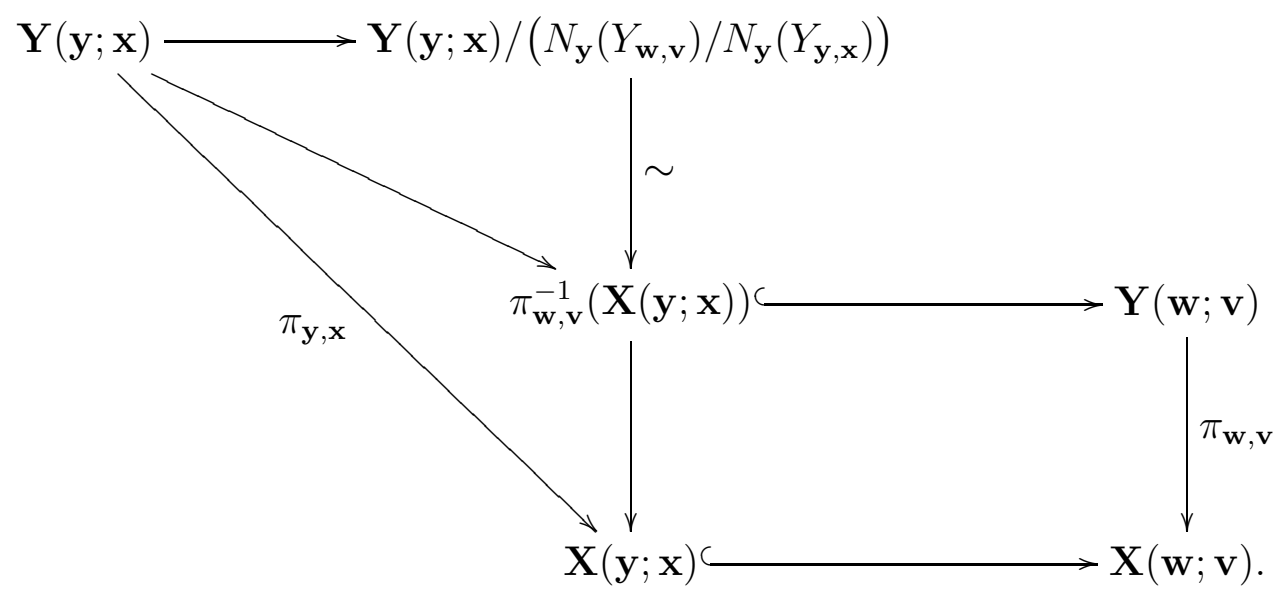

6.4. Remarques. Pour construire le revêtement $\pi_{\mathbf{w}, \mathbf{v}}: \mathbf{Y}(\mathbf{w} ; \mathbf{v}) \rightarrow \mathbf{X}(\mathbf{w} ; \mathbf{v})$, il a été nécessaire de passer par un groupe $\hat{\mathbf{G}}$ dont les coracines sont injectives et tel qu'il existe un tore central $F$-stable $\mathbf{C}$ de $\hat{\mathbf{G}}$ tel que $\mathbf{G} \simeq \hat{\mathbf{G}} / \mathbf{C}$. Disons dans ce paragraphe qu'une paire $(\hat{\mathbf{G}}, \mathbf{C})$ vérifiant ces propriétés est sympathique.

Le lecteur pourra vérifier que, dans le cas où $\mathbf{G}=\mathbf{P G L}_{2}(\mathbf{F})$ et où $p \neq 2$, alors on ne peut effectuer directement la construction dans $\mathbf{G}$. En effet, on obtiendrait ainsi un revêtement connexe de $\mathbf{Y}(s ; 1)$ (ici, $s$ désigne l'unique réflexion simple de $W$ ), alors que l'on attend un revêtement non connexe. Il est donc nécessaire de passer par $\hat{\mathbf{G}}=\mathbf{G L}_{2}(\mathbf{F})$.

Soient $\left(\hat{\mathbf{G}}_{1}, \mathbf{C}_{1}\right)$ et $\left(\hat{\mathbf{G}}_{2}, \mathbf{C}_{2}\right)$ deux paires sympathiques et $\hat{\mathbf{G}}_{0}$ le produit fibré de $\hat{\mathbf{G}}_{1}$ et $\hat{\mathbf{G}}_{2}$ au-dessus de $\mathbf{G}$. On note $\hat{\mathbf{Y}}_{i}(\mathbf{w} ; \mathbf{v})$ la variété définie dans $\hat{\mathbf{G}}_{i}$ comme $\mathbf{Y}(\mathbf{w} ; \mathbf{v})$ a été définie dans $\mathbf{G}$ lorsque les coracines de $\mathbf{G}$ sont injectives. La paire $\left(\hat{\mathbf{G}}_{0}, \mathbf{C}_{1} \times \mathbf{C}_{2}\right)$ est sympathique et $\hat{\mathbf{Y}}_{i}(\mathbf{w} ; \mathbf{v}) \stackrel{\sim}{\rightarrow} \hat{\mathbf{Y}}_{0}(\mathbf{w} ; \mathbf{v}) / \mathbf{C}_{j}^{F}$, où $\{i, j\}=\{1,2\}$. Cela fournit un isomorphisme $\hat{\mathbf{Y}}_{1}(\mathbf{w} ; \mathbf{v}) / \mathbf{C}_{1}^{F} \simeq \hat{\mathbf{Y}}_{2}(\mathbf{w} ; \mathbf{v}) / \mathbf{C}_{2}^{F}$. On en déduit que la variété $\mathbf{Y}(\mathbf{w} ; \mathbf{v})$ ne dépend pas du choix de $\hat{\mathbf{G}}$, à isomorphisme unique près. 


\section{Calculs de monodromie}

Nous allons appliquer ici les résultats du paragraphe précédent au calcul des images directes supérieures (dans une compactification lisse adaptée) des faisceaux localement constants sur les variétés $\mathbf{X}(\mathbf{w})$ provenant du revêtement étale $\mathbf{Y}(\dot{\mathbf{w}}) \rightarrow \mathbf{X}(\mathbf{w})$. Le résultat (le théorème 7.7), généralise [DeLu, lemme 9.13]. Nous obtiendrons comme conséquences des résultats sur les foncteurs de Deligne-Lusztig (cf $\S 8.2$ ).

\section{Dans toute cette section, nous supposons que $\Lambda$ est un corps.}

On fixe dans cette section une suite w d'éléments de $\bar{S}$.

Soient $\mathbf{v}, \mathbf{x}$ et $\mathbf{y}$ des suites d'éléments de $\bar{S}$ telles que $\mathbf{v} \leq \mathbf{x} \leq \mathbf{y} \leq \mathbf{w}$. Nous noterons $j_{\mathbf{y} ; \mathbf{x}}^{\mathbf{w} ;}: \mathbf{X}(\mathbf{y} ; \mathbf{x}) \hookrightarrow \mathbf{X}(\mathbf{w} ; \mathbf{v})$ l'injection canonique. Pour simplifier, nous remplacerons, lorsque $\mathbf{y}=\mathbf{x}$, l'indice ou l'exposant $\mathbf{y} ; \mathbf{x}$ par $\mathbf{y}$. D'autre part, nous posons

$$
\begin{aligned}
\mathbf{X}(\overline{\mathbf{x}}) & =\coprod_{\mathbf{x}^{\prime} \leq \mathbf{x}} \mathbf{X}\left(\mathbf{x}^{\prime}\right) \\
( & =\mathbf{X}(\mathbf{x} ;(1, \ldots, 1)) \quad) .
\end{aligned}
$$

Si $\mathbf{v}=(1, \ldots, 1)$, nous remplacerons l'indice ou l'exposant $\mathbf{x} ; \mathbf{v}$ par $\overline{\mathbf{x}}$. Par exemple, $j_{\mathbf{y}}^{\overline{\mathbf{x}}}$ désigne l'injection canonique $\mathbf{X}(\mathbf{y}) \hookrightarrow \mathbf{X}(\overline{\mathbf{x}})$.

La compactification de $\mathbf{X}(\mathbf{x})$ ainsi construite possède de bonnes propriétés [DeLu, lemme 9.11] :

Lemme 7.1. La variété $\mathbf{X}(\overline{\mathbf{x}})$ est projective lisse et le complémentaire de $\mathbf{X}(\mathbf{x})$ est un diviseur à croisements normaux $\bigcup_{\mathbf{v}} \mathbf{X}(\overline{\mathbf{v}})$ où $\mathbf{v}$ décrit l'ensemble des suites telles que $\mathbf{v} \leq \mathbf{x}$ et $l(\mathbf{x})-l(\mathbf{v})=1$.

Nous noterons

$$
\mathcal{F}^{\mathbf{y} ; \mathbf{x}}: D^{b}\left(\Lambda \mathbf{T}^{\mathbf{y} F} / N_{\mathbf{y}}\left(Y_{\mathbf{y}, \mathbf{x}}\right)\right) \rightarrow D_{\Lambda}^{b}(\mathbf{X}(\mathbf{y} ; \mathbf{x}))
$$

le foncteur $\mathcal{F}_{\mathbf{T}^{\mathbf{y}} F / N_{\mathbf{y}}\left(Y_{\mathbf{y} ; \mathbf{x}}\right)}^{\mathbf{Y}(\mathbf{y} ; \mathbf{x}}$ défini au $\S 3.2$. Nous fixons aussi dans cette section un caractère linéaire $\theta: \mathbf{T}^{\mathbf{w} F} \rightarrow \Lambda^{\times}$. Si $\mathbf{w}_{\theta} \leq \mathbf{x} \leq \mathbf{y} \leq \mathbf{w}$, il résulte de $\S 4.4 .2$ que $\theta$ définit un caractère linéaire $\theta_{\mathbf{y} ; \mathbf{x}}$ du groupe $\mathbf{T}^{\mathbf{y} F} / N_{\mathbf{y}}\left(Y_{\mathbf{y}, \mathbf{x}}\right)$ (pour la définition de $\mathbf{w}_{\theta}$, voir $\S 4.4 .2$ ). Nous noterons $\mathcal{F}_{\theta}^{\mathbf{y} ; \mathbf{x}}$ le faisceau localement constant $\mathcal{F}^{\mathbf{y} ; \mathbf{x}}\left(\Lambda_{\theta_{\mathbf{y} ; \mathbf{x}}}\right)$ sur la variété $\mathbf{X}(\mathbf{y} ; \mathbf{x})$. Compte tenu du corollaire 6.8 et de la proposition $4.11(3), \mathcal{F}_{\theta}^{\mathbf{y} ; \mathbf{x}}$ est la restriction de $\mathcal{F}_{\theta}^{\mathbf{w} ; \mathbf{w}_{\theta}}$ à $\mathbf{X}(\mathbf{y} ; \mathbf{x})$. En d'autres termes,

$$
\mathcal{F}_{\theta}^{\mathbf{y} ; \mathbf{x}} \simeq\left(j_{\mathbf{y} ; \mathbf{x}}^{\mathbf{w} ; \mathbf{w}_{\theta}}\right)^{*} \mathcal{F}_{\theta}^{\mathbf{w} ; \mathbf{w}_{\theta}}
$$

Comme expliqué dans l'introduction de cette section, nous allons calculer ici les faisceaux $\mathrm{R}^{i}\left(j_{\mathrm{w}}^{\overline{\mathbf{w}}}\right)_{*} \mathcal{F}_{\theta}^{\mathrm{w}}$. La proposition suivante combine un résultat fondamental de Deligne et Lusztig [DeLu, lemme 9.13] et une conséquence immédiate de la construction des variétés $\mathbf{Y}(\mathbf{w} ; \mathbf{v})$.

Proposition 7.3. Soient $\mathbf{v}, \mathbf{v}^{\prime}$ et $\mathbf{x}$ des suites d'éléments de $\bar{S}$ telles que $\mathbf{w}_{\theta} \leq \mathbf{v} \leq \mathbf{x}$ et $\mathbf{v}^{\prime}<\mathbf{v}$. 
(1) Si $\mathbf{v}$ est le plus petit élément tel que $\mathbf{v}^{\prime} \leq \mathbf{v}$ et $\mathbf{w}_{\theta} \leq \mathbf{v}$, alors le faisceau $\mathcal{F}_{\theta}^{\mathbf{x}}$ se ramifie le long de chaque composante du diviseur $\mathbf{X}\left(\mathbf{x} ; \mathbf{v}^{\prime}\right)-\mathbf{X}(\mathbf{x} ; \mathbf{v})$ et le morphisme canonique $\left(j_{\mathbf{x} ; \mathbf{v}}^{\mathbf{x} ; \mathbf{v}^{\prime}}\right) ! \mathcal{F}_{\theta}^{\mathbf{x} ; \mathbf{v}} \stackrel{\sim}{\rightarrow} \mathrm{R}\left(j_{\mathbf{x} ; \mathbf{v}}^{\mathbf{x} ; \mathbf{v}^{\prime}}\right)_{*} \mathcal{F}_{\theta}^{\mathbf{x} ; \mathbf{v}}$ est un isomorphisme.

(2) Si $\mathbf{w}_{\theta} \leq \mathbf{v}^{\prime}$, alors le faisceau $\mathcal{F}_{\theta}^{\mathbf{x}}$ ne se ramifie pas le long du diviseur $\mathbf{X}\left(\mathbf{x} ; \mathbf{v}^{\prime}\right)-$ $\mathbf{X}(\mathbf{x} ; \mathbf{v})$.

Démonstration. On a

$$
\mathbf{X}\left(\mathbf{x} ; \mathbf{v}^{\prime}\right)-\mathbf{X}(\mathbf{x} ; \mathbf{v})=\bigcup_{\mathbf{x}^{\prime}} \mathbf{X}\left(\mathbf{x}^{\prime} ; \mathbf{v}^{\prime}\right)
$$

où $\mathbf{v}^{\prime} \leq \mathbf{x}^{\prime} \leq \mathbf{x}, \mathbf{v} \not \leq \mathbf{x}^{\prime}$ et $l\left(\mathbf{x}^{\prime}\right)=l(\mathbf{x})-1$.

Sous l'hypothèse de (1), on a $\mathbf{w}_{\theta} \not \mathbf{x} \mathbf{x}^{\prime}$. D'après [DeLu, lemme 9.13] (la preuve, faite pour des $\overline{\mathbf{Q}}_{\ell^{-}}$faisceaux reste valable pour des $\Lambda$-faisceaux), le faisceau $\mathcal{F}_{\theta}^{\mathbf{x}}$ se ramifie le long du diviseur $\mathbf{X}\left(\overline{\mathbf{x}^{\prime}}\right)$ de $\overline{\mathbf{X}}(\mathbf{x})$, i.e., la restriction de $\left(j_{\mathbf{x}}^{\overline{\mathbf{x}}}\right)_{*} \mathcal{F}_{\theta}^{\mathbf{x}}$ à $\mathbf{X}\left(\overline{\mathbf{x}^{\prime}}\right)$ est nulle. On en déduit que $\mathcal{F}_{\theta}^{\mathbf{x} ; \mathbf{v}}$ se ramifie le long du diviseur $\mathbf{X}\left(\mathbf{x}^{\prime} ; \mathbf{v}^{\prime}\right)$. Par conséquent, le morphisme canonique $\left(j_{\mathbf{x} ; \mathbf{v}}^{\mathbf{x} ; \mathbf{v}^{\prime}}\right) ! \mathcal{F}_{\theta}^{\mathbf{x} ; \mathbf{v}} \rightarrow\left(j_{\mathbf{x} ; \mathbf{v}}^{\mathbf{x} ; \mathbf{v}^{\prime}}\right)_{*} \mathcal{F}_{\theta}^{\mathbf{x} ; \mathbf{v}}$ est un isomorphisme. L'annulation $\operatorname{des} \mathrm{R}^{i}\left(j_{\mathbf{x} ; \mathbf{v}}^{\mathbf{x} ; \mathbf{v}^{\prime}}\right)_{*} \mathcal{F}_{\theta}^{\mathbf{x} ; \mathbf{v}}$ pour $i>0$ résulte de [SGA $4 \frac{1}{2}$, exemple 1.19 .1 p.180] (on utilise ici le lemme 7.1 et la propriété de $\mathcal{F}_{\theta}^{\mathbf{x} ; \mathbf{v}}$ d'être modérément ramifié), d'où (1).

(2) découle directement de 7.2.

Définissons maintenant quelques sous-catégories de $D_{\Lambda}^{b}(\mathbf{X}(\overline{\mathbf{w}}))$. Prenons $\mathbf{w}_{\theta} \leq \mathbf{y}$. On pose

$$
\begin{aligned}
A_{\leq \mathbf{y}}^{1}(\theta) & =\left\{\left(j_{\mathbf{x}}^{\overline{\mathbf{w}}}\right)_{!} \mathcal{F}_{\theta}^{\mathbf{x}} \mid \mathbf{w}_{\theta} \leq \mathbf{x} \leq \mathbf{y}\right\}, \\
A_{<\mathbf{y}}^{1}(\theta) & =\left\{\left(j_{\mathbf{x}}^{\overline{\mathbf{w}}}\right)_{!} \mathcal{F}_{\theta}^{\mathbf{x}} \mid \mathbf{w}_{\theta} \leq \mathbf{x}<\mathbf{y}\right\}, \\
A_{\leq \mathbf{y}}^{2}(\theta) & =\left\{\left(j_{\mathbf{x} ; \mathbf{x}^{\prime}}^{\overline{\mathbf{w}}}\right)_{!} \mathcal{F}_{\theta}^{\mathbf{x} ; \mathbf{x}^{\prime}} \mid \mathbf{w}_{\theta} \leq \mathbf{x}^{\prime} \leq \mathbf{x} \leq \mathbf{y}\right\}, \\
A_{<\mathbf{y}}^{2}(\theta) & =\left\{\left(j_{\mathbf{x} ; \mathbf{x}^{\prime}}^{\overline{\mathbf{w}}}\right)_{!} \mathcal{F}_{\theta}^{\mathbf{x} ; \mathbf{x}^{\prime}} \mid \mathbf{w}_{\theta} \leq \mathbf{x}^{\prime} \leq \mathbf{x}<\mathbf{y}\right\}, \\
A_{\leq \mathbf{y}}^{3}(\theta) & =\left\{\left(j_{\mathbf{x} ; \mathbf{w}_{\theta}}^{\overline{\mathbf{w}}}\right)_{!} \mathcal{F}_{\theta}^{\mathbf{x} ; \mathbf{w}_{\theta}} \mid \mathbf{w}_{\theta} \leq \mathbf{x} \leq \mathbf{y}\right\}, \\
A_{<\mathbf{y}}^{3}(\theta) & =\left\{\left(j_{\mathbf{x} ; \mathbf{w}_{\theta}}^{\overline{\mathbf{w}}}\right)_{!} \mathcal{F}_{\theta}^{\mathbf{x} ; \mathbf{w}_{\theta}} \mid \mathbf{w}_{\theta} \leq \mathbf{x}<\mathbf{y}\right\}, \\
A_{\leq \mathbf{y}}^{4}(\theta) & =\left\{\mathrm{R}\left(j_{\mathbf{x}}^{\overline{\mathbf{w}}}\right)_{*} \mathcal{F}_{\theta}^{\mathbf{x}} \mid \mathbf{w}_{\theta} \leq \mathbf{x} \leq \mathbf{y}\right\}, \\
A_{<\mathbf{y}}^{4}(\theta) & =\left\{\mathrm{R}\left(j_{\mathbf{x}}^{\overline{\mathbf{w}}}\right)_{*} \mathcal{F}_{\theta}^{\mathbf{x}} \mid \mathbf{w}_{\theta} \leq \mathbf{x}<\mathbf{y}\right\}, \\
A_{\leq \mathbf{y}}^{5}(\theta) & =\left\{\mathrm{R}\left(j_{\mathbf{x} ; \mathbf{x}^{\prime}}^{\overline{\mathbf{w}}}\right)_{*} \mathcal{F}_{\theta}^{\mathbf{x} ; \mathbf{x}^{\prime}} \mid \mathbf{w}_{\theta} \leq \mathbf{x} \mathbf{x}^{\prime} \leq \mathbf{x} \leq \mathbf{y}\right\}, \\
A_{<\mathbf{y}}^{5}(\theta) & =\left\{\mathrm{R}\left(j_{\mathbf{x} ; \mathbf{x}^{\prime}}^{\overline{\mathbf{w}}}\right)_{*} \mathcal{F}_{\theta}^{\mathbf{x} ; \mathbf{x}^{\prime}} \mid \mathbf{w}_{\theta} \leq \mathbf{x} \mathbf{x}^{\prime} \leq \mathbf{x}<\mathbf{y}\right\}, \\
A_{\leq \mathbf{y}}^{6}(\theta) & =\left\{\mathrm{R}\left(j_{\mathbf{x} ; \mathbf{w}_{\theta}}^{\overline{\mathbf{w}}}\right)_{*} \mathcal{F}_{\theta}^{\mathbf{x} ; \mathbf{w}_{\theta}} \mid \mathbf{w}_{\theta} \leq \mathbf{x} \leq \mathbf{y}\right\}, \\
\text { et } \quad A_{<\mathbf{y}}^{6}(\theta) & =\left\{\mathrm{R}\left(j_{\mathbf{x} ; \mathbf{w}_{\theta}}^{\overline{\mathbf{w}}}\right)_{*} \mathcal{F}_{\theta}^{\mathbf{x} ; \mathbf{w}_{\theta}} \mid \mathbf{w}_{\theta} \leq \mathbf{x}<\mathbf{y}\right\} .
\end{aligned}
$$

Si $(i, *) \in\{1,2,3,4,5,6\} \times\{\leq \mathbf{y},<\mathbf{y}\}$, nous noterons $\mathcal{A}_{*}^{i}(\theta)$ la sous-catégorie de $D_{\Lambda}^{b}(\mathbf{X}(\overline{\mathbf{w}}))$ engendrée par $A_{*}^{i}(\theta)$. 
Proposition 7.4. Pour $* \in\{\leq \mathbf{y},<\mathbf{y}\}$, on $a$

$$
\mathcal{A}_{*}^{1}(\theta)=\mathcal{A}_{*}^{2}(\theta)=\mathcal{A}_{*}^{3}(\theta)=\mathcal{A}_{*}^{4}(\theta)=\mathcal{A}_{*}^{5}(\theta)=\mathcal{A}_{*}^{6}(\theta) .
$$

Démonstration. Notons pour commencer que le morphisme canonique

$$
\left(j_{\mathbf{x} ; \mathbf{w}_{\theta}}^{\overline{\mathbf{w}}}\right)_{!} \mathcal{F}_{\theta}^{\mathbf{x} ; \mathbf{w}_{\theta}} \stackrel{\sim}{\rightarrow} \mathrm{R}\left(j_{\mathbf{x} ; \mathbf{w}_{\theta}}^{\overline{\mathbf{w}}}\right)_{*} \mathcal{F}_{\theta}^{\mathbf{x} ; \mathbf{w}_{\theta}}
$$

est un isomorphisme (proposition $7.3(1)$ ), donc $\mathcal{A}_{*}^{3}(\theta)=\mathcal{A}_{*}^{6}(\theta)$.

Notons que $\mathcal{A}_{*}^{1}(\theta) \subset \mathcal{A}_{*}^{2}(\theta)$ et $\mathcal{A}_{*}^{3}(\theta) \subset \mathcal{A}_{*}^{2}(\theta)$.

Prenons $\mathbf{w}_{\theta} \leq \mathbf{v}^{\prime} \leq \mathbf{v} \leq \mathbf{x} \leq \mathbf{w}$ avec $l(\mathbf{v})=l\left(\mathbf{v}^{\prime}\right)+1$. Soit $\mathbf{x}^{\prime} \leq \mathbf{x}$ tel que $\mathbf{v}^{\prime} \leq \mathbf{x}^{\prime}, \mathbf{v} \not \leq \mathbf{x}^{\prime}$ et $l\left(\mathbf{x}^{\prime}\right)=l(\mathbf{x})-1$. On a $\mathbf{X}\left(\mathbf{x} ; \mathbf{v}^{\prime}\right)-\mathbf{X}(\mathbf{x} ; \mathbf{v})=\mathbf{X}\left(\mathbf{x}^{\prime} ; \mathbf{v}^{\prime}\right)$, d'où on déduit une suite exacte

$$
0 \rightarrow\left(j_{\mathbf{x} ; \mathbf{v}}^{\overline{\mathbf{w}}}\right) ! \mathcal{F}_{\theta}^{\mathbf{x} ; \mathbf{v}} \rightarrow\left(j_{\mathbf{x} ; \mathbf{v}^{\prime}}^{\overline{\mathbf{w}}}\right) ! \mathcal{F}_{\theta}^{\mathbf{x} ; \mathbf{v}^{\prime}} \rightarrow\left(j_{\mathbf{x}^{\prime} ; \mathbf{v}^{\prime}}^{\overline{\mathbf{w}}}\right) ! \mathcal{F}_{\theta}^{\mathbf{x}^{\prime} ; \mathbf{v}^{\prime}} \rightarrow 0 .
$$

Par conséquent, $\left(j_{\mathbf{x} ; \mathbf{v}^{\prime}}^{\overline{\mathbf{w}}}\right) ! \mathcal{F}_{\theta}^{\mathbf{x} ; \mathbf{v}^{\prime}}$ est dans la sous-catégorie engendrée par les $\left(j_{\mathbf{x}^{\prime \prime} ; \mathbf{v}^{\prime \prime}}^{\overline{\mathbf{w}}}\right) ! \mathcal{F}_{\theta}^{\mathbf{x}^{\prime \prime} ; \mathbf{v}^{\prime \prime}}$, où $l\left(\mathbf{x}^{\prime \prime}\right)-l\left(\mathbf{v}^{\prime \prime}\right)<l(\mathbf{x})-l\left(\mathbf{v}^{\prime}\right)$ et $\mathbf{w}_{\theta} \leq \mathbf{v}^{\prime \prime} \leq \mathbf{x}^{\prime \prime} \leq \mathbf{x}$. Par récurrence, on en déduit que $\mathcal{A}_{*}^{2}(\theta) \subset \mathcal{A}_{*}^{1}(\theta)$.

On déduit aussi de la suite exacte (1) que $\left(j_{\mathbf{x} ; \mathbf{v}}\right)_{!} \mathcal{F}_{\theta}^{\mathbf{x} ; \mathbf{v}}$ est dans la sous-catégorie engendrée par les $\left(j_{\mathbf{x}^{\prime \prime}}^{\overline{\mathbf{w}} ; \mathbf{v}^{\prime \prime}}\right) ! \mathcal{F}_{\theta}^{\mathbf{x}^{\prime \prime} ; \mathbf{v}^{\prime \prime}}$, où $\mathbf{v}^{\prime \prime}<\mathbf{v}$ et $\mathbf{x}^{\prime \prime} \leq \mathbf{x}$. Par récurrence, on en déduit que $\mathcal{A}_{*}^{2}(\theta) \subset \mathcal{A}_{*}^{3}(\theta)$.

On a donc démontré que $\mathcal{A}_{*}^{1}(\theta)=\mathcal{A}_{*}^{2}(\theta)=\mathcal{A}_{*}^{3}(\theta)$.

Le théorème de pureté cohomologique [Mi, chapitre VI, théorème 5.1] fournit un triangle distingué

$$
\mathcal{F}_{\theta}^{\mathbf{x} ; \mathbf{v}^{\prime}} \rightarrow \mathrm{R}\left(j_{\mathbf{x} ; \mathbf{v}}^{\mathbf{x} ; \mathbf{v}^{\prime}}\right)_{*} \mathcal{F}_{\theta}^{\mathbf{x} ; \mathbf{v}} \rightarrow\left(j_{\mathbf{x}^{\prime} ; \mathbf{v}^{\prime}}^{\mathbf{x} ; \mathbf{v}^{\prime}}\right) ! \mathcal{F}_{\theta}^{\mathbf{x}^{\prime} ; \mathbf{v}^{\prime}}[-1] \rightsquigarrow
$$

d'où

$$
\mathrm{R}\left(j_{\mathbf{x} ; \mathbf{v}^{\prime}}^{\overline{\mathbf{w}}}\right)_{*} \mathcal{F}_{\theta}^{\mathbf{x} ; \mathbf{v}^{\prime}} \rightarrow \mathrm{R}\left(j_{\mathbf{x} ; \mathbf{v}}^{\overline{\mathbf{w}}}\right)_{*} \mathcal{F}_{\theta}^{\mathbf{x} ; \mathbf{v}} \rightarrow \mathrm{R}\left(j_{\mathbf{x}^{\prime} ; \mathbf{v}^{\prime}}^{\overline{\mathbf{w}}}\right)_{*} \mathcal{F}_{\theta}^{\mathbf{x}^{\prime} ; \mathbf{v}^{\prime}}[-1] \rightsquigarrow .
$$

On prouve alors, comme précédemment, que $\mathcal{A}_{*}^{4}(\theta)=\mathcal{A}_{*}^{5}(\theta)=\mathcal{A}_{*}^{6}(\theta)$.

Remarque 7.5. Notons que la dualité de Verdier échange les catégories $\mathcal{A}_{*}^{i}(\theta)$ et $\mathcal{A}_{*}^{i}\left(\theta^{-1}\right)$.

Corollaire 7.6. Le cône du morphisme canonique de complexes $\left(j_{\mathrm{w}}^{\overline{\mathrm{w}}}\right)_{!} \mathcal{F}_{\theta}^{\mathrm{w}} \longrightarrow \mathrm{R}\left(j_{\mathrm{w}}^{\overline{\mathrm{w}}}\right)_{*} \mathcal{F}_{\theta}^{\mathrm{w}}$ est dans la sous-catégorie $\mathcal{A}_{<\mathbf{w}}^{i}(\theta)$ pour tout $i \in\{1,2,3,4,5,6\}$.

Démonstration. D'après la proposition 7.4 , ce cône appartient à la catégorie $\mathcal{A}_{<\mathbf{w}}^{1}(\theta)$. D'autre part, son support est contenu dans le complémentaire de $\mathbf{X}(\mathbf{w})$ dans $\mathbf{X}(\overline{\mathbf{w}})$, d'où le résultat.

Théorème 7.7. Soit $i$ un entier naturel. Alors

$$
\left(j_{\mathbf{v}}^{\overline{\mathbf{w}}}\right)^{*}\left(\mathrm{R}^{i}\left(j_{\mathbf{w}}^{\overline{\mathbf{w}}}\right)_{*} \mathcal{F}_{\theta}^{\mathbf{w}}\right) \simeq \begin{cases}\left.\left(\mathcal{F}_{\theta}^{\mathbf{v}}\right)^{\oplus(l(\mathbf{w})-l(\mathbf{v})}\right) & \text { si } \mathbf{v} \in \mathcal{I}(\mathbf{w}, \theta), \\ 0 & \text { sinon. }\end{cases}
$$


Démonstration. Factorisons l'immersion ouverte $j_{\mathbf{w}}^{\overline{\mathbf{w}}}$ de la manière suivante

$$
\mathbf{X}(\mathbf{w}) \stackrel{j_{1}}{\longrightarrow} \mathbf{X}\left(\mathbf{w} ; \mathbf{w}_{\theta}\right) \stackrel{j_{2}}{\longrightarrow} \overline{\mathbf{X}}(\mathbf{w}) .
$$

D'après la proposition 7.4, le support du complexe $\mathrm{R}\left(j_{\mathbf{w}}^{\overline{\mathbf{w}}}\right)_{*} \mathcal{F}_{\theta}^{\mathbf{w}}$ est contenu dans $\mathbf{X}\left(\mathbf{w} ; \mathbf{w}_{\theta}\right)$, ce qui montre que

$$
\mathrm{R}\left(j_{\mathbf{w}}^{\overline{\mathbf{w}}}\right)_{*} \mathcal{F}_{\theta}^{\mathbf{w}} \simeq\left(j_{2}\right) ! \mathrm{R}\left(j_{1}\right)_{*} \mathcal{F}_{\theta}^{\mathbf{w}}
$$

Mais, $\mathcal{F}_{\theta}^{\mathbf{w}} \stackrel{\sim}{\rightarrow} j_{1}^{*} \mathcal{F}_{\theta}^{\mathbf{w} ; \mathbf{w}_{\theta}}$, par conséquent, d'après le lemme 12.2 ,

$$
\mathrm{R}\left(j_{1}\right)_{*} \mathcal{F}_{\theta}^{\mathbf{w}} \stackrel{\sim}{\rightarrow}\left(\mathrm{R}\left(j_{1}\right)_{*} \Lambda_{\mathbf{X}(\mathbf{w})}\right) \otimes_{\Lambda} \mathcal{F}_{\theta}^{\mathbf{w} ; \mathbf{w}_{\theta}} .
$$

Le théorème 7.7 découle alors immédiatement de (1) et (2), en appliquant les lemmes 7.1 et 12.7 .

\section{Induction De Deligne-Lusztig et SÉRIEs RAtionnelles}

Nous allons appliquer les résultats de la section précédente aux foncteurs de Lusztig. Pour cela, nous ferons l'hypothèse suivante.

8.1. Orthogonalité. Soient $(\mathbf{w}, \theta)$ et $\left(\mathbf{w}^{\prime}, \theta^{\prime}\right)$ deux éléments de $\boldsymbol{\nabla}(\mathbf{T}, W, F)$.

Le résultat suivant généralise [DeLu, théorème 6.2] qui considère le cas $\Lambda=K$ et la conjugaison géométrique.

Théorème 8.1. Si $(\mathbf{w}, \theta)$ et $\left(\mathbf{w}^{\prime}, \theta^{\prime}\right)$ sont dans deux séries rationnelles différentes, alors

$$
\left(\mathcal{R}_{\dot{\mathbf{w}}} \Lambda \mathbf{T}^{\mathbf{w} F} e_{\theta^{-1}}\right)^{\mathrm{op}} \otimes_{\Lambda \mathbf{G}^{F}}^{\mathrm{L}} \mathcal{R}_{\dot{\mathbf{w}}^{\prime}} \Lambda \mathbf{T}^{\mathbf{w}^{\prime} F} e_{\theta^{\prime}}=0 .
$$

Démonstration. Par la formule des coefficients universels, il suffit de démontrer le théorème lorsque $\Lambda$ est un corps, ce que nous supposerons dans la suite. Commençons par démontrer la proposition lorsque le centre de $\mathbf{G}$ est connexe (lorsque $\Lambda=K$, c'est [DeLu, théorème $6.2])$. Dans ce cas, $(\mathbf{w}, \theta)$ et $\left(\mathbf{w}^{\prime}, \theta^{\prime}\right)$ ne sont pas géométriquement conjugués (cf remarque $4.3)$.

D'après le lemme 3.2 et la proposition 5.7 , on a un isomorphisme dans $D^{b}\left(\Lambda\left(\mathbf{T}^{\mathbf{w} F} \times\right.\right.$ $\left.\left.\mathbf{T}^{\prime} F\right)\right)$

$$
\mathrm{R} \Gamma_{c}\left(\left(\mathbf{Y}(\dot{\mathbf{w}}) \times \mathbf{Y}^{\prime}\left(\dot{\mathbf{w}}^{\prime}\right)\right) / \Delta\left(\mathbf{G}^{F}\right)\right) \stackrel{\sim}{\rightarrow} \mathrm{R} \Gamma_{c}(\mathbf{Y}(\dot{\mathbf{w}}))^{\mathrm{op}} \otimes_{\Lambda \mathbf{G}^{F}}^{\mathrm{L}} \mathrm{R} \Gamma_{c}\left(\mathbf{Y}\left(\dot{\mathbf{w}}^{\prime}\right)\right) .
$$

La preuve est alors similaire à celle de [DeLu, théorème 6.2] : on se ramène à prouver l'assertion suivante.

Soit $\mathbf{X}$ une variété munie d'une action d'un groupe algébrique $\mathbf{H}$ contenant $\mathbf{T}^{\mathbf{w} F} \times$ $\mathbf{T}^{\mathbf{w}^{\prime} F}$. On suppose le caractère $\theta^{-1} \times \theta^{\prime}$ non trivial sur $Q=\left(\mathbf{T}^{\mathbf{w} F} \times \mathbf{T}^{\mathbf{w}^{\prime} F}\right) \cap \mathbf{H}^{\circ}$. Alors, $\left(e_{\theta^{-1}} \otimes_{\Lambda} e_{\theta^{\prime}}\right) \mathrm{R} \Gamma_{c}(\mathbf{X})=0$.

Démontrons donc cette assertion. D'après [DeLu, proposition 6.4], le groupe connexe $\mathbf{H}^{\circ}$ agit trivialement sur $H_{c}^{*}(\mathbf{X})$. Puisque $\operatorname{Res}_{Q}^{\mathbf{T}^{\mathbf{w} F} \times \mathbf{T}^{\mathbf{w}^{\prime} F}}\left(\theta^{-1} \times \theta^{\prime}\right)$ n'est pas dans le bloc principal de $\Lambda Q$, on a

$$
\mathrm{RHom}_{\Lambda Q}^{\bullet}\left(\Lambda \mathbf{T}^{\mathbf{w} F} e_{\theta^{-1}} \otimes_{\Lambda} \Lambda \mathbf{T}^{\mathbf{w}^{\prime} F} e_{\theta^{\prime}}, \mathrm{R} \Gamma_{c}(\mathbf{X})\right)=0
$$


donc

$$
\mathrm{RHom}_{\Lambda\left(\mathbf{T}^{\mathbf{w} F} \times \mathbf{T}^{\left.\mathbf{w}^{\prime} F\right)}\right.}^{\bullet}\left(\operatorname{Ind}_{Q}^{\mathbf{T}^{\mathbf{w} F} \times \mathbf{T}^{\mathbf{w}^{\prime} F}}\left(\Lambda \mathbf{T}^{\mathbf{w} F} e_{\theta^{-1}} \otimes_{\Lambda} \Lambda \mathbf{T}^{\mathbf{w}^{\prime} F} e_{\theta^{\prime}}\right), \mathrm{R} \Gamma_{c}(\mathbf{X})\right)=0
$$

et a fortiori

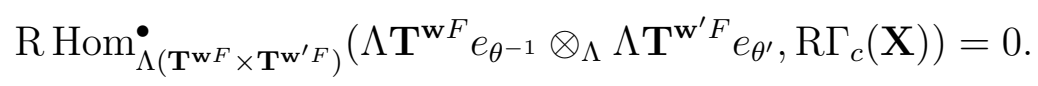

Revenons maintenant à $\mathbf{G}$ quelconque. Soit $A$ (respectivement $A^{\prime}$ ) l'ensemble des caractères linéaires de $\tilde{\mathbf{T}}^{\mathbf{w} F}$ (respectivement $\tilde{\mathbf{T}}^{\mathbf{w}^{\prime} F}$ ) étendant $\theta$ (respectivement $\theta^{\prime}$ ).

Le complexe

$$
C=\left(\mathcal{R}_{\dot{\mathbf{w}}}\left(\Lambda \mathbf{T}^{\mathbf{w} F} e_{\theta^{-1}}\right)\right)^{\mathrm{op}} \otimes_{\Lambda \mathbf{G}^{F}}^{\mathrm{L}} \mathcal{R}_{\dot{\mathbf{w}}^{\prime}}\left(\Lambda \mathbf{T}^{\mathbf{w}^{\prime} F} e_{\theta^{\prime}}\right)
$$

est un facteur direct du complexe

$$
\tilde{C}=\left(\operatorname{Ind}_{\mathbf{G}^{F}}^{\tilde{\mathbf{G}}^{F}} \mathcal{R}_{\dot{\mathbf{w}}}\left(\Lambda \mathbf{T}^{\mathbf{w} F} e_{\theta^{-1}}\right)\right)^{\mathrm{op}} \otimes_{\Lambda \tilde{\mathbf{G}}^{F}}^{\mathrm{L}}\left(\operatorname{Ind}_{\mathbf{G}^{F}}^{\tilde{\mathbf{G}}^{F}} \mathcal{R}_{\dot{\mathbf{w}}^{\prime}}\left(\Lambda \mathbf{T}^{\mathbf{w}^{\prime} F} e_{\theta^{\prime}}\right)\right) .
$$

De plus, d'après (5.14), on a

et

$$
\begin{aligned}
& \operatorname{Ind}_{\mathbf{G}^{F}}^{\tilde{\mathbf{G}}^{F}} \mathcal{R}_{\dot{\mathbf{w}}}\left(\Lambda \mathbf{T}^{\mathbf{w} F} e_{\theta^{-1}}\right) \simeq \tilde{\mathcal{R}}_{\dot{\mathbf{w}}}\left(\Lambda \tilde{\mathbf{T}}^{\mathbf{w} F} e_{\theta^{-1}}\right) \\
& \operatorname{Ind}_{\mathbf{G}^{F}}^{\tilde{\mathbf{G}}^{F}} \mathcal{R}_{\dot{\mathbf{w}}^{\prime}}\left(\Lambda \mathbf{T}^{\mathbf{w}^{\prime} F} e_{\theta^{\prime}}\right) \simeq \tilde{\mathcal{R}}_{\dot{\mathbf{w}}}\left(\Lambda \tilde{\mathbf{T}}^{\mathbf{w}^{\prime} F} e_{\theta^{\prime}}\right) .
\end{aligned}
$$

Mais

$$
\begin{aligned}
& \Lambda \tilde{\mathbf{T}}^{\mathbf{w} F} e_{\theta^{-1}}=\bigoplus_{\tilde{\theta} \in A} \Lambda \tilde{\mathbf{T}}^{\mathbf{w} F} e_{\tilde{\theta}^{-1}} \\
& \Lambda \tilde{\mathbf{T}}^{\mathbf{w}^{\prime} F} e_{\theta^{\prime}}=\bigoplus_{\tilde{\theta}^{\prime} \in A^{\prime}} \Lambda \tilde{\mathbf{T}}^{\mathbf{w}^{\prime} F} e_{\tilde{\theta}^{\prime}} .
\end{aligned}
$$

Par conséquent,

$$
\tilde{C} \simeq \bigoplus_{\substack{\tilde{\theta} \in A \\ \tilde{\theta^{\prime}} \in A^{\prime}}}\left(\tilde{\mathcal{R}}_{\mathbf{w}}\left(\Lambda \tilde{\mathbf{T}}^{\mathbf{w} F} e_{\tilde{\theta}^{-1}}\right)\right)^{\mathrm{op}} \otimes_{\Lambda \tilde{\mathbf{G}}^{F}}^{\mathrm{L}} \tilde{\mathcal{R}}_{\dot{\mathbf{w}}^{\prime}}\left(\Lambda \tilde{\mathbf{T}}^{\mathbf{w}^{\prime} F} e_{\tilde{\theta}^{\prime}}\right)
$$

Puisque $(\mathbf{w}, \theta)$ et $\left(\mathbf{w}^{\prime}, \theta^{\prime}\right)$ ne sont pas dans la même série rationnelle, les paires $(\mathbf{w}, \tilde{\theta})$ et $\left(\mathbf{w}^{\prime}, \tilde{\theta}^{\prime}\right)$ ne sont pas géométriquement conjuguées dans $\tilde{\mathbf{G}}$ pour tous $\tilde{\theta} \in A$ et $\tilde{\theta}^{\prime} \in A^{\prime}$. On a alors $\tilde{C}=0$ d'après la première partie de la preuve, ce qui implique que $C=0$.

Corollaire 8.2. Si $(\mathbf{w}, \theta)$ et $\left(\mathbf{w}^{\prime}, \theta^{\prime}\right)$ appartiennent à deux séries rationnelles différentes de $\boldsymbol{\nabla}(\mathbf{T}, W, F)$, alors

$$
\left(\mathcal{R}_{\dot{\mathbf{w}}} \Lambda_{\theta^{-1}}\right)^{\mathrm{op}} \otimes_{\Lambda \mathbf{G}^{F}}^{\mathrm{L}} \mathcal{R}_{\dot{\mathbf{w}}^{\prime}} \Lambda_{\theta^{\prime}}=0
$$


8.2. Profondeur. Dans toute cette section §8.2, nous supposerons que $\Lambda$ est un corps.

En appliquant le foncteur $\mathrm{R} \Gamma(\mathbf{X}(\overline{\mathbf{w}}),-)$ à l'énoncé du corollaire 7.6, on obtient :

Théorème 8.3. Soit $\mathbf{w} \in \Sigma(\bar{S})$ et $\theta$ un caractère linéaire de $\mathbf{T}^{\mathbf{w} F}$. Alors, le cône du morphisme canonique $\mathcal{R}_{\mathbf{w}} \Lambda_{\theta} \rightarrow \mathcal{S}_{\dot{\mathbf{w}}} \Lambda_{\theta}$ est dans la sous-catégorie triangulée de $D^{b}\left(\Lambda \mathbf{G}^{F}\right)$ engendrée par les complexes $\mathcal{R}_{\dot{\mathbf{x}}} \Lambda_{\theta_{\mathbf{x}}}$ où $\mathbf{x} \in \mathcal{I}(\mathbf{w}, \theta)$ et $\mathbf{x}<\mathbf{w}$.

Corollaire 8.4. Soit $\mathbf{w} \in \Sigma(\bar{S})$ et $\theta$ un caractère linéaire de $\mathbf{T}^{\mathbf{w} F}$. Alors, le cône du morphisme canonique $\mathcal{R}_{\mathbf{w}} \Lambda_{\theta} \rightarrow \mathcal{S}_{\mathbf{w}} \Lambda_{\theta}$ est dans la sous-catégorie triangulée de $D^{b}\left(\Lambda \mathbf{G}^{F}\right)$ engendrée par les complexes $\mathcal{R}_{\dot{\mathbf{x}}} \Lambda_{\theta^{\prime}}$ où $\left(\mathbf{x}, \theta^{\prime}\right) \equiv_{W}(\mathbf{w}, \theta)$ et $\mathbf{x}<\mathbf{w}$.

Démonstration. Le corollaire découle du théorème 8.3 et du corollaire 4.6.

Corollaire 8.5. Si $(\mathbf{w}, \theta)$ et $\left(\mathbf{w}^{\prime}, \theta^{\prime}\right)$ appartiennent à deux séries rationnelles différentes de $\boldsymbol{\nabla}(\mathbf{T}, W, F)$, alors

$$
\mathrm{RHom}_{\Lambda \mathbf{G}^{F}}\left(\mathcal{S}_{\mathbf{w}^{\mathbf{w}}} \Lambda \mathbf{T}^{\mathbf{w} F} e_{\theta}, \mathcal{S}_{\mathbf{w}^{\prime}} \Lambda \mathbf{T}^{\mathbf{w}^{\prime} F} e_{\theta^{\prime}}\right)=0 .
$$

Démonstration. La proposition 5.4 permet de se ramener au cas où $\mathbf{w}, \mathbf{w}^{\prime} \in \Sigma(\bar{S})$.

On a un isomorphisme

$$
\mathrm{RHom}_{\Lambda \mathbf{G}^{F}}^{\bullet}\left(\mathcal{S}_{\mathbf{w}} \Lambda \mathbf{T}^{\mathbf{w} F} e_{\theta}, \mathcal{S}_{\dot{\mathbf{w}}^{\prime}} \Lambda \mathbf{T}^{\mathbf{w}^{\prime} F} e_{\theta^{\prime}}\right) \simeq\left(\mathcal{S}_{\mathbf{w}} \Lambda \mathbf{T}^{\mathbf{w} F} e_{\theta}\right)^{*} \otimes_{\Lambda \mathbf{G}^{F}}^{\mathrm{L}} \mathcal{S}_{\mathbf{w}^{\prime}} \Lambda \mathbf{T}^{\mathbf{w}^{\prime} F} e_{\theta^{\prime}}
$$

D'après (5.11), on a

$$
\left(\mathcal{S}_{\mathbf{w}} \Lambda \mathbf{T}^{\mathbf{w} F} e_{\theta}\right)^{*} \simeq\left(\mathcal{R}_{\dot{\mathbf{w}}} \Lambda \mathbf{T}^{\mathbf{w} F} e_{\theta^{-1}}\right)^{\mathrm{op}}[2 l(\mathbf{w})]
$$

Le corollaire 8.5 résulte alors du théorème 8.1 et du fait que $\mathcal{S}_{\dot{\mathbf{w}}^{\prime}} \Lambda \mathrm{T}^{\mathbf{w}^{\prime} F} e_{\theta^{\prime}}$ est dans la catégorie engendrée par les $\mathcal{R}_{\dot{\mathbf{x}}} \Lambda \mathbf{T}^{\mathbf{x} F} e_{\theta_{1}}$, où $\left(\mathbf{x}, \theta_{1}\right)$ appartient à la même série rationnelle que $\left(\mathbf{w}^{\prime}, \theta^{\prime}\right)$ (corollaire 8.4).

On a un accouplement parfait

$$
\begin{array}{ccc}
<,>: \quad \mathrm{K}\left(\Lambda \mathbf{G}^{F}\right) \times \mathrm{K}\left(\Lambda \mathbf{G}^{F} \text {-proj }\right) & \longrightarrow & \mathbf{Z} \\
([M],[P]) & \longmapsto & \operatorname{dim}\left(\operatorname{Hom}_{\Lambda \mathbf{G}^{F}}(P, M)\right) .
\end{array}
$$

D'après [DeLu, proposition 7.5], on a

$$
|W| \cdot\left[\Lambda \mathbf{G}^{F}\right]=\sum_{w \in W}(-1)^{l(w)} \frac{\left|\mathbf{G}^{F}\right|_{p^{\prime}}}{\left|\mathbf{T}^{w F}\right|}\left[\mathcal{R}_{(\dot{w})} \Lambda \mathbf{T}^{w F}\right] .
$$

En appliquant (5.11), on obtient

$$
|W| \cdot\left[\Lambda \mathbf{G}^{F}\right]=\sum_{w \in W}(-1)^{l(w)} \frac{\left|\mathbf{G}^{F}\right|_{p^{\prime}}}{\left|\mathbf{T}^{w F}\right|}\left[\mathcal{S}_{(\dot{w})} \Lambda \mathbf{T}^{w F}\right] .
$$

Par conséquent, si $M$ est un $\Lambda \mathbf{G}^{F}$-module simple, il existe $((\dot{w}), \theta) \in \boldsymbol{\nabla}(\mathbf{T}, W, F)$ tel que

$$
<[M],\left[\mathcal{S}_{(\dot{w})} \Lambda \mathbf{T}^{w F} e_{\theta}\right]>\neq 0 .
$$

On a donc : 
Lemme 8.8. Si $M$ est un $\Lambda \mathbf{G}^{F}$-module simple, il existe $(\mathbf{w}, \theta) \in \boldsymbol{\nabla}(\mathbf{T}, W, F)$ tel que

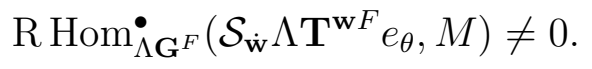

Remarque 8.9. Il serait souhaitable de trouver une preuve plus directe de ce lemme.

Proposition 8.10. Si $M$ est un $\Lambda \mathbf{G}^{F}$-module simple et si $\mathbf{w}$ est une suite d'éléments de $W$ avec $l(\mathbf{w})$ minimal telle que

$$
\mathrm{R} \operatorname{Hom}_{\Lambda \mathbf{G}^{F}}\left(\mathcal{S}_{\mathbf{w}} \Lambda \mathbf{T}^{\mathbf{w} F}, M\right) \neq 0,
$$

alors la cohomologie du complexe $\mathrm{R}_{\operatorname{Hom}_{\Lambda \mathbf{G}^{F}}}\left(\mathcal{S}_{\mathbf{w}} \Lambda \mathbf{T}^{\mathbf{w} F}, M\right)$ est concentrée en degré $-l(\mathbf{w})$.

Démonstration. La proposition 5.4 permet de se ramener au cas où $\mathbf{w} \in \Sigma(\bar{S})$. D'après (5.11), on a

$$
\mathrm{R} \operatorname{Hom}_{\Lambda \mathbf{G}^{F}}\left(\mathcal{S}_{\dot{\mathbf{w}}} \Lambda \mathbf{T}^{\mathbf{w} F}, M\right) \simeq\left(\mathcal{R}_{\dot{\mathbf{w}}} \Lambda \mathbf{T}^{\mathbf{w} F}\right)^{\mathrm{op}} \otimes_{\Lambda \mathbf{G}^{F}}^{\mathrm{L}} M[2 l(\mathbf{w})] .
$$

Cela montre que, si $\mathbf{v}<\mathbf{w}$, alors

$$
\left(\mathcal{R}_{\dot{\mathbf{v}}} \Lambda \mathbf{T}^{\mathbf{v} F}\right)^{\mathrm{op}} \otimes_{\Lambda \mathbf{G}^{F}}^{\mathrm{L}} M=0 .
$$

Donc, pour tout caractère linéaire $\theta: \mathbf{T}^{\mathbf{v} F} \rightarrow \Lambda^{\times}$, on a

$$
\left(\mathcal{R}_{\dot{\mathbf{v}}} \Lambda_{\theta}\right)^{\mathrm{op}} \otimes_{\Lambda \mathbf{G}^{F}}^{\mathrm{L}} M=0 .
$$

Mais, d'après le théorème 8.3 , le cône du morphisme canonique $\mathcal{R}_{\dot{\mathrm{w}}} \Lambda \mathbf{T}^{\mathbf{w} F} \rightarrow \mathcal{S}_{\dot{\mathrm{w}}} \Lambda \mathrm{T}^{\mathbf{w} F}$ est dans la sous-catégorie engendrée par les $\mathcal{R}_{\dot{\mathbf{v}}} \Lambda_{\theta}$ où $\mathbf{v}<\mathbf{w}$ et $\theta$ est un caractère linéaire de $\mathbf{T}^{\mathbf{v} F}$. Par suite, le morphisme

$$
\left(\mathcal{R}_{\dot{\mathbf{w}}} \Lambda \mathbf{T}^{\mathbf{w} F}\right)^{\mathrm{op}} \otimes_{\Lambda \mathbf{G}^{F}}^{\mathrm{L}} M \longrightarrow\left(\mathcal{S}_{\dot{\mathbf{w}}} \Lambda \mathbf{T}^{\mathbf{w} F}\right)^{\mathrm{op}} \otimes_{\Lambda \mathbf{G}^{F}}^{\mathrm{L}} M
$$

est un isomorphisme. La preuve de la proposition 8.10 est alors complétée par le lemme 12.6 et la proposition 5.7.

Remarque 8.11. Comme nous l'a fait remarquer Marc Cabanes, on peut en fait démontrer directement que $\mathrm{R} \operatorname{Hom}_{\Lambda \mathrm{G}^{F}}\left(\mathcal{S}_{\mathbf{w}} \Lambda \mathbf{T}^{\mathrm{w} F},\left(M^{*}\right)^{\text {op }}\right) \neq 0$. En effet,

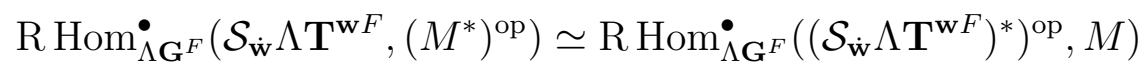

car $\mathcal{S}_{\dot{\mathrm{w}}} \Lambda \mathrm{T}^{\mathrm{w} F}$ est parfait. Maintenant, la dualité de Poincaré fournit

$$
\left.\left.\mathrm{R} \mathrm{Hom}_{\Lambda \mathbf{G}^{F}}\left(\left(\mathcal{S}_{\dot{\mathbf{w}}} \Lambda \mathbf{T}^{\mathbf{w} F}\right)^{*}\right)^{\mathrm{op}}, M\right) \simeq \operatorname{RHom}_{\mathbf{\Lambda G}^{F}}\left(\mathcal{R}_{\dot{\mathbf{w}}} \Lambda \mathbf{T}^{\mathbf{w} F}\right), M\right)
$$

d'où le résultat annoncé. Par conséquent, l'application du lemme 12.6 se fait dans un cas où $M$ et $\left(M^{*}\right)^{\text {op }}$ vérifient les hypothèses. Le résultat d'Haastert (cf remarque 12.4) suffit alors.

La proposition suivante fournit plusieurs caractérisations d'un élément $\mathbf{w}$ de longueur minimale :

Proposition 8.12. Soit $M$ un $\Lambda \mathbf{G}^{F}$-module simple et soit $(\mathbf{w}, \theta) \in \boldsymbol{\nabla}(\mathbf{T}, W, F)$. Les propriétés suivantes sont équivalentes :

(a) $(\mathbf{w}, \theta)$ est de longueur minimale tel que $<[M],\left[\mathcal{R}_{\mathfrak{w}} \Lambda \mathbf{T}^{\mathbf{w} F} e_{\theta}\right]>\neq 0$;

(b) $(\mathbf{w}, \theta)$ est de longueur minimale tel que $\mathrm{R} \mathrm{Hom}_{\Lambda \mathbf{G}^{F}}\left(\mathcal{R}_{\mathbf{w}} \Lambda \mathbf{T}^{\mathbf{w} F} e_{\theta}, M\right) \neq 0$; 


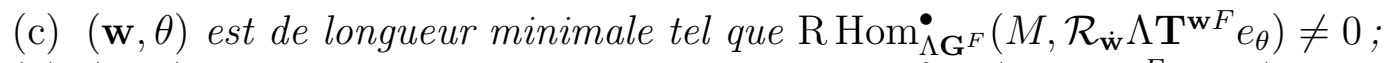

(d) $(\mathbf{w}, \theta)$ est de longueur minimale tel que $\mathrm{R} \mathrm{Hom}_{\Lambda \mathbf{G}^{F}}\left(\mathcal{S}_{\mathbf{w}} \Lambda \mathbf{T}^{\mathbf{w} F} e_{\theta}, M\right) \neq 0$;

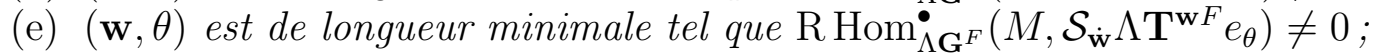

(f) Un énoncé parmi $(a),(b),(c),(d)$ ou $(e)$ en remplaçant e $e_{\theta}$ par $e_{\theta^{-1}}$ et $M$ par $\left(M^{*}\right)^{\mathrm{op}}$.

Démonstration. D'après le corollaire 8.4, le cône du morphisme canonique $\mathcal{R}_{\mathbf{w}} \Lambda \mathrm{T}^{\mathbf{w} F} e_{\theta} \rightarrow$ $\mathcal{S}_{\mathbf{w}} \Lambda \mathbf{T}^{\mathbf{w} F} e_{\theta}$ est dans la sous-catégorie engendrée par les $\mathcal{R}_{\mathbf{w}} \Lambda \mathbf{T}^{\mathbf{w}^{\prime} F}$ avec $\mathbf{w}^{\prime}<\mathbf{w}$. Ceci démontre l'équivalence de (b) et (d) et de (c) et (e).

Soit $C$ un complexe borné de $\Lambda \mathbf{G}^{F}$-modules projectifs, sans facteur direct non nul homotope à 0 . Alors, $\mathrm{R}_{\operatorname{Hom}^{\bullet}}(C, M) \neq 0$ si et seulement si $\mathrm{R} \operatorname{Hom}^{\bullet}(M, C) \neq 0$, si et seulement si une enveloppe projective de $M$ est facteur direct d'une des composantes de $C$. Cette remarque démontre l'équivalence entre (b) et (c) et entre (d) et (e).

L'équivalence entre (a) et (d) résulte de la proposition 8.10. L'équivalence avec les énoncés de (f) résulte de (5.11).

Remarque 8.13. Il n'est pas difficile de voir que si w vérifie les propriétés de la proposition, alors $\sigma(\mathbf{w})$ est dans $\sigma(W)$ et son image dans $W$ est de longueur minimale dans sa classe de $F$-conjugaison (car $\left[\mathcal{R}_{\dot{\mathbf{w}}} \Lambda \mathrm{T}^{\mathbf{w} F} e_{\theta}\right]$ ne dépend que de la classe de $F$-conjugaison de l'image de $\mathbf{w}$ dans $W$ ). Il est sans doute vrai que l'image de $\mathbf{w}$ dans $W$ est dans une $F$-classe de conjugaison de $W$ ne dépendant que de $M$. C'est un résultat connu lorsque $\Lambda=K$ [DiMiRo]. La preuve, qui requiert l'étude des valeurs propres de l'endomorphisme de Frobenius sur le cohomologie, ne semble pas s'étendre à $\Lambda=k$.

\section{EnGENDREMEnt DE $\Lambda \mathbf{G}^{F}$-parf}

9.1. Engendrement de catégories de complexes parfaits. Soit $A$ une algèbre libre de type fini sur un anneau local complet $\mathcal{O}$, d'idéal maximal $\mathfrak{m}$ et de corps résiduel $\overline{\mathcal{O}}$.

Soit $E$ un ensemble fini de complexes parfaits de $A$, muni d'une relation de préordre partiel.

Lemme 9.1. Supposons que pour tout $A$-module simple $M$, il existe $C \in E$ tel que $\mathrm{R} \mathrm{Hom}_{D^{b}\left(A \otimes_{\mathcal{O}} \overline{\mathcal{O}}\right)}\left(C \otimes_{\mathcal{O}}^{\mathrm{L}} \overline{\mathcal{O}}, M\right) \neq 0$ et que si $C$ est minimal avec cette propriété, alors la cohomologie du complexe $\mathrm{R} \mathrm{Hom}_{D^{b}\left(A \otimes_{\mathcal{O}} \overline{\mathcal{O}}\right)}^{\bullet}\left(C \otimes_{\mathcal{O}}^{\mathrm{L}} \overline{\mathcal{O}}, M\right)$ est concentrée en degré 0.

Alors, A-parf est engendrée par E.

Démonstration. Nous noterons $\bar{C}=C \otimes_{\mathcal{O}}^{\mathrm{L}} \overline{\mathcal{O}}$ pour $C$ un complexe de $A$-modules. Soit $C \in E$. On note $\mathcal{S}(\leq C)$ (respectivement $\mathcal{S}(<C)$ ) l'ensemble des classes d'isomorphisme de $A$-modules simples $M$ tels qu'il existe $C^{\prime} \in E$ tel que $C^{\prime} \leq C$ (respectivement $C^{\prime}<C$ ) et $\mathrm{RHom}^{\bullet}\left(\bar{C}^{\prime}, M\right) \neq 0$.

Quitte à remplacer les éléments de $E$ par des complexes quasi-isomorphes, on peut supposer, et on le fera, que ce sont des complexes bornés de modules projectifs sans facteurs directs non nuls homotopes à 0 . Alors, pour $C \in E$ et pour $M$ simple, on a $\mathrm{RHom}^{\bullet}(\bar{C}, M[i]) \neq 0$ si et seulement si une enveloppe projective $P_{M}$ de $M$ est facteur 
direct de $C^{-i}$. Lorsque ces conditions équivalentes sont vérifiées, on a $M \in \mathcal{S}(\leq C)$. Lorsqu'en plus $i \neq 0$, alors $M \in \mathcal{S}(<C)$.

Soit $\langle E\rangle$ la sous-catégorie de $K^{b}(A$-proj) engendrée par $E$.

Considérons l'assertion suivante :

$\left(\star_{C}\right)$ Pour $M \in \mathcal{S}(\leq C)$, alors $P_{M}$ est dans $\langle E\rangle$.

Notons que le lemme 9.1 découle immédiatement de $\left(\star_{C}\right)$ pour tout $C$. Nous allons montrer $\left(\star_{C}\right)$ par récurrence sur $C$.

Soit $C$ un complexe minimal. Alors, $\mathrm{R} \mathrm{Hom}^{\bullet}(\bar{C}, M[i])=0$ pour tout module simple $M$ et tout entier non nul $i$. Par conséquent, $C$ n'a qu'un terme non nul, $C^{0}$, qui est un $A$-module projectif de type fini. Pour $M$ un $A$-module simple, alors $\operatorname{Hom}\left(C^{0}, M\right) \neq 0$ si et seulement si $P_{M}$ est facteur direct de $C^{0}$. On en déduit immédiatement $\left(\star_{C}\right)$.

Prenons maintenant $C \in E$ tel que $\left(\star_{C^{\prime}}\right)$ est vraie pour tout $C^{\prime}<C$. Les facteurs directs indécomposables de $C^{i}$ sont de la forme $P_{M}$ avec $M \in \mathcal{S}(<C)$ pour $i \neq 0$, donc il sont dans $\langle E\rangle$. On en déduit donc que $C^{0}$ est dans $\langle E\rangle$. Par conséquent, $P_{M}$ est dans $\langle E\rangle$ pour $M \in \mathcal{S}(C)$, ce qui montre $\left(\star_{C}\right)$.

On se donne maintenant une relation d'équivalence $\sim \operatorname{sur} E$.

Proposition 9.2. On garde les hypothèses du lemme 9.1 et on suppose en plus que $\mathrm{R} \mathrm{Hom}^{\bullet}\left(C \otimes_{\mathcal{O}}^{\mathrm{L}} \overline{\mathcal{O}}, C^{\prime} \otimes_{\mathcal{O}}^{\mathrm{L}} \overline{\mathcal{O}}\right)=0$ pour tous $C, C^{\prime} \in E$ avec $C \not C^{\prime}$.

Soit $\langle X\rangle$ la sous-catégorie de A-parf engendrée par $X \in E / \sim$. Alors, on a une décomposition

$$
A \text {-parf }=\bigoplus_{X \in E / \sim}\langle X\rangle .
$$

Par conséquent, pour $X \in E / \sim$, il existe un idempotent central $e_{X}$ de $A$ tel que $\langle X\rangle=$ $A e_{X}$-parf et on a une décomposition de l'unité en somme d'idempotents orthogonaux

$$
1=\sum_{X \in E / \sim}^{\perp} e_{X} .
$$

Démonstration. Pour $C$ et $C^{\prime}$ deux complexes parfaits de $A$-modules, le morphisme canonique $\operatorname{R~Hom}^{\bullet}\left(C, C^{\prime}\right) \otimes_{\mathcal{O}}^{\mathrm{L}} \overline{\mathcal{O}} \stackrel{\sim}{\rightarrow} \mathrm{R} \mathrm{Hom}^{\bullet}\left(C \otimes_{\mathcal{O}}^{\mathrm{L}} \overline{\mathcal{O}}, C^{\prime} \otimes_{\mathcal{O}}^{\mathrm{L}} \overline{\mathcal{O}}\right)$ est un isomorphisme. Par conséquent, la nullité de $\operatorname{RHom}^{\bullet}\left(C, C^{\prime}\right)$ équivaut à celle de $\operatorname{RHom}^{\bullet}\left(C \otimes_{\mathcal{O}}^{\mathrm{L}} \overline{\mathcal{O}}, C^{\prime} \otimes_{\mathcal{O}}^{\mathrm{L}} \overline{\mathcal{O}}\right)$.

D'après le lemme 9.1, la réunion des $\langle X\rangle$, où $X \in E / \sim$, engendre $A$-parf. Puisque ces sous-catégories sont deux à deux orthogonales, la catégorie qu'elles engendrent est leur somme directe.

9.2. Décomposition en séries. Nous allons démontrer que la catégorie $\Lambda \mathbf{G}^{F}$-parf est engendrée par les complexes $\left(\mathcal{R}_{\mathbf{w}} \Lambda \mathbf{T}^{\mathbf{w} F}\right)_{\mathbf{w} \in \Sigma(W)}$ et obtenir une décomposition de cette catégorie paramétrée par les séries rationnelles. Pour cela, il nous suffira d'appliquer la proposition 9.2, la vérification des hypothèses résultant du travail effectué dans le $\S 8$.

Si $\mathcal{X}$ est une série rationnelle de $\boldsymbol{\nabla}(\mathbf{T}, W, F)$, nous noterons $\mathcal{X}_{\min }$ l'ensemble des couples $(w, \theta)$ où $w \in W$ et $\theta$ est un caractère linéaire de $\mathbf{T}^{w F}$ vérifiant la propriété suivante : il 
existe un $\Lambda \mathbf{G}^{F}$-module simple $M$ tel que $((\dot{w}), \theta)$ est un élément de longueur minimale de $\boldsymbol{\nabla}(\mathbf{T}, W, F)$ vérifiant

$$
\mathrm{RHom}_{\Lambda \mathbf{G}^{F}}\left(\mathcal{S}_{\dot{w}} \Lambda \mathbf{T}^{w F} e_{\theta}, M\right) \neq 0 .
$$

Il résulte de la remarque 8.13 que si $(w, \theta) \in \mathcal{X}_{\min }$, alors $w$ est de longueur minimale dans sa classe de $F$-conjugaison.

Théorème A. Soit $\mathcal{X}$ une série rationnelle dans $\boldsymbol{\nabla}(\mathbf{T}, W, F)$. Alors, il existe un idempotent central $e_{\mathcal{X}}=e_{\mathcal{X}}^{\mathbf{G}^{F}}$ de $\Lambda \mathbf{G}^{F}$ tel que chacune des familles suivantes engendre la catégorie $\Lambda \mathbf{G}^{F} e_{\mathcal{X}}$-parf :

$-\left\{\mathcal{R}_{\dot{\mathbf{w}}} \Lambda \mathbf{T}^{\mathbf{w} F} e_{\theta}\right\}_{(\mathbf{w}, \theta) \in \mathcal{X}}$

- $\left\{\mathcal{S}_{\dot{\mathrm{w}}} \Lambda \mathbf{T}^{\mathbf{w} F} e_{\theta}\right\}_{(\mathbf{w}, \theta) \in \mathcal{X}}$

$-\left\{\mathcal{R}_{(w)} \Lambda \mathbf{T}^{w F} e_{\theta}\right\}_{(w, \theta) \in \mathcal{X}_{\min }}$

- $\left\{\mathcal{S}_{(\dot{w})} \Lambda \mathbf{T}^{w F} e_{\theta}\right\}_{(w, \theta) \in \mathcal{X}_{\text {min }}}$

On a une décomposition de 1 en somme d'idempotents centraux deux à deux orthogonaux

$$
1=\sum_{\mathcal{X} \in \boldsymbol{\nabla}(\mathbf{T}, W, F) / \equiv_{W}}^{\perp} e_{\mathcal{X}}
$$

Démonstration. Soit $E$ l'ensemble des complexes $\mathcal{S}_{(\dot{w})} \Lambda \mathbf{T}^{w F} e_{\theta}[l(w)]$, où $(w, \theta) \in \mathcal{X}_{\text {min }}$ et $\mathcal{X}$ décrit les séries rationnelles. On pose $(w, \theta) \leq\left(w^{\prime}, \theta^{\prime}\right)$ si $w \leq w^{\prime}$.

D'après le lemme 8.8 et la proposition 8.12 , pour tout $\Lambda G^{F}$-module simple $M$, il existe $C \in E$ tel que $\mathrm{RHom}^{\bullet}(C, M) \neq 0$. D'après la proposition 8.10, si $C$ est de longueur minimale avec cette propriété, alors $\mathrm{R} \mathrm{Hom}^{\bullet}(C, M)$ est concentré en degré 0 .

On introduit la relation d'équivalence sur $E$ donnée par $(w, \theta) \sim\left(w^{\prime}, \theta^{\prime}\right)$ si $((w), \theta)$ et $\left(\left(w^{\prime}\right), \theta^{\prime}\right)$ sont rationnellement conjugués. Alors, $\mathrm{R} \operatorname{Hom}^{\bullet}\left(C, C^{\prime}\right)=0$ si $C \nsim C^{\prime}$ d'après le corollaire 8.5.

Il résulte alors de la proposition 9.2 que $\left\{\mathcal{S}_{(\dot{w})} \Lambda \mathbf{T}^{w F} e_{\theta}\right\}_{(w, \theta) \in \mathcal{X}_{\min }}$ engendre $\Lambda \mathbf{G}^{F} e_{\mathcal{X}}$-parf. On montre de même que $\left\{\mathcal{S}_{\mathbf{w}} \Lambda \mathbf{T}^{\mathbf{w} F} e_{\theta}\right\}_{(\mathbf{w}, \theta) \in \mathcal{X}}$ engendre $\Lambda \mathbf{G}^{F} e_{\mathcal{X}}$-parf.

L'isomorphisme (5.11) (dualité de Poincaré) montre les assertions concernant les complexes $\mathcal{R}_{(\dot{w})} \Lambda \mathbf{T}^{w F} e_{\theta}$ et $\mathcal{R}_{\mathbf{w}} \Lambda \mathbf{T}^{\mathbf{w} F} e_{\theta}$.

Remarque 9.3. Considérons tout d'abord le cas où $\Lambda=K$. La catégorie $K \mathbf{G}^{F}$-mod étant semi-simple, la catégorie $K \mathbf{G}^{F}$-parf est équivalente à la catégorie des $K \mathbf{G}^{F}$-modules gradués.

Pour $\mathcal{X} \in \nabla_{K}(\mathbf{T}, W, F) / \equiv_{W}$, on note $\mathcal{E}\left(\mathbf{G}^{F}, \mathcal{X}\right)$ l'ensemble des caractères irréductibles $\chi$ de $\mathbf{G}^{F}$ tels qu'il existe $(\mathbf{w}, \theta) \in \mathcal{X}$ avec $<\chi,{ }_{K} \mathrm{R}_{\dot{\mathbf{w}}} \theta>\neq 0$. Il résulte du théorème $A$ que

$$
\operatorname{Irr}_{K} \mathbf{G}^{F}=\coprod_{\mathcal{X} \in \nabla_{K}(\mathbf{T}, W, F) / \equiv_{W}} \mathcal{E}\left(\mathbf{G}^{F}, \mathcal{X}\right)
$$

et que

$$
e_{\mathcal{X}}=\sum_{\chi \in \mathcal{E}\left(\mathbf{G}^{F}, \mathcal{X}\right)} e_{\chi}
$$


où $e_{\chi}$ désigne l'unique idempotent primitif central de $K \mathbf{G}^{F}$ tel que $\chi\left(e_{\chi}\right) \neq 0$. On retrouve ainsi la décomposition de [DeLu].

Le morphisme canonique $R \rightarrow k$ induit une bijection $\boldsymbol{\nabla}_{R}(\mathbf{T}, W, F) \stackrel{\sim}{\rightarrow} \boldsymbol{\nabla}_{k}(\mathbf{T}, W, F), \mathcal{X} \mapsto$ $\overline{\mathcal{X}}$, compatible avec les séries rationnelles. Pour $\mathcal{X} \in \boldsymbol{\nabla}_{R}(\mathbf{T}, W, F) / \equiv_{W}$, l'image de $e_{\mathcal{X}} \in R \mathbf{G}^{F}$ dans $k \mathbf{G}^{F}$ est $e_{\overline{\mathcal{X}}}$.

Par ailleurs, nous avons une application surjective $\boldsymbol{\nabla}_{K}(\mathbf{T}, W, F) \rightarrow \boldsymbol{\nabla}_{k}(\mathbf{T}, W, F)$ qui est obtenue par passage au quotient $R \rightarrow k$ (en effet, un caractère linéaire $\mathbf{T}^{\mathbf{w} F} \rightarrow K^{\times}$est à valeurs dans $\left.R^{\times}\right)$. Elle induit une application surjective $\boldsymbol{\nabla}_{K}(\mathbf{T}, W, F) \rightarrow \boldsymbol{\nabla}_{R}(\mathbf{T}, W, F)$ en composant avec la bijection décrite précédemment, d'où finalement une application surjective entre séries rationnelles $\boldsymbol{\nabla}_{K}(\mathbf{T}, W, F) / \equiv_{W} \rightarrow \boldsymbol{\nabla}_{R}(\mathbf{T}, W, F) / \equiv_{W}, \mathcal{X} \mapsto \mathcal{X}_{\ell^{\prime}}$. Cette notation rappelle que cette application envoie un $\operatorname{couple}(\mathbf{w}, \theta)$ sur le couple $\left(\mathbf{w}, \theta_{\ell^{\prime}}\right)$, où $\theta_{\ell^{\prime}}$ désigne la $\ell^{\prime}$-partie du caractère linéaire $\theta$. Fixons une série rationnelle $\mathcal{X}_{0} \in$ $\boldsymbol{\nabla}_{R}(\mathbf{T}, W, F) / \equiv_{W}$ et posons

$$
\mathcal{E}_{\ell}\left(\mathbf{G}^{F}, \mathcal{X}_{0}\right)=\coprod_{\mathcal{X}_{\ell^{\prime}}=\mathcal{X}_{0}} \mathcal{E}\left(\mathbf{G}^{F}, \mathcal{X}\right) .
$$

On a alors

$$
e_{\mathcal{X}_{0}}=\sum_{\mathcal{X}_{\ell^{\prime}}=\mathcal{X}_{0}} e_{\mathcal{X}}=\sum_{\chi \in \mathcal{E}_{\ell}\left(\mathbf{G}^{F}, \mathcal{X}_{0}\right)} e_{\chi} \in R \mathbf{G}^{F}
$$

Nous retrouvons la compatibilité des séries avec les blocs établie par Broué et Michel [BrMi, théorème 2.2].

\section{Foncteurs De Lusztig}

10.1. Définition. Fixons une partie $I$ de $\Delta$. Nous notons $W_{I}$ le sous-groupe de $W$ engendré par la famille $\left(s_{\alpha}\right)_{\alpha \in I}, \mathbf{P}_{I}$ le sous-groupe parabolique $\mathbf{B} W_{I} \mathbf{B}$ de $\mathbf{G}, \mathbf{V}_{I}$ le radical unipotent de $\mathbf{P}_{I}$ et $\mathbf{L}_{I}$ le complément de Levi de $\mathbf{V}_{I}$ dans $\mathbf{P}_{I}$ contenant $\mathbf{T}$. Le groupe de Weyl de $\mathbf{L}_{I}$ relatif à $\mathbf{T}$ est alors égal à $W_{I}$. Nous notons aussi $\mathbf{B}_{I}$ le sous-groupe de Borel de $\mathbf{L}_{I}$ égal à $\mathbf{B} \cap \mathbf{L}_{I}$ et $\mathbf{U}_{I}$ son radical unipotent. Avec ces notations, on a ${ }^{F} \boldsymbol{?}_{I}=\boldsymbol{?}_{\phi(I)}$ si ? désigne l'une des lettres $\mathbf{B}, \mathbf{L}, \mathbf{P}, \mathbf{U}, \mathbf{V}$ ou $W$.

Fixons maintenant un élément $v$ de $W$ tel que $v \phi(I)=I$. Alors, $\dot{v} F$ normalise $\mathbf{L}_{I}$ et on pose

$$
\mathbf{Y}_{I, v}=\left\{g \mathbf{V}_{I} \in \mathbf{G} / \mathbf{V}_{I} \mid g^{-1 F} g \in \mathbf{V}_{I} \dot{v}^{F} \mathbf{V}_{I}\right\}
$$

Le groupe fini $\mathbf{G}^{F}$ agit par multiplication à gauche sur la variété $\mathbf{Y}_{I, v}$. De même, le groupe $\mathbf{L}_{I F}^{\dot{v} F}$ agit par multiplication à droite. Munie de ces deux actions, $\mathbf{Y}_{I, v}$ est une $\mathbf{G}^{F}$-variété$\mathbf{L}_{I}^{i v}$ de dimension $l(v)$. Elle est régulière en tant que variété- $\mathbf{L}_{I}^{i F}$. On définit alors un foncteur entre catégories dérivées

$$
\begin{aligned}
& \mathcal{R}_{I, v}: D^{b}\left(\Lambda \mathbf{L}_{I}^{\dot{v} F}\right) \quad \longrightarrow \quad D^{b}\left(\Lambda \mathbf{G}^{F}\right) \\
& C \quad \longmapsto R \Gamma_{c}\left(\mathbf{Y}_{I, v}\right) \otimes_{\Lambda \mathbf{L}_{I}^{i F}}^{\mathrm{L}} C .
\end{aligned}
$$

Avec les notations du $\S 3.2$, on a $\mathcal{R}_{I, v}=\mathcal{R}_{\mathbf{L}_{I}^{i f}}^{\mathbf{G}^{F}}\left(\mathbf{Y}_{I, v}\right)$. Posons maintenant

$$
\mathbf{X}_{I, v}=\left\{g \mathbf{P}_{I} \in \mathbf{G} / \mathbf{P}_{I} \mid g^{-1}{ }^{F} g \in \mathbf{P}_{I} \dot{v}^{F} \mathbf{P}_{I}\right\} .
$$


Alors, d'après la proposition 5.1 appliquée à $\mathbf{P}=\mathbf{P}_{I}, \mathbf{H}=\mathbf{L}=\mathbf{L}_{I}, n=\dot{v}$ et $\mathbf{H}^{\prime}=1$, l'application naturelle

$$
\pi_{I, v}: \begin{aligned}
\mathbf{Y}_{I, v} & \longrightarrow \mathbf{X}_{I, v} \\
g \mathbf{V}_{I} & \longmapsto g \mathbf{P}_{I}
\end{aligned}
$$

induit un isomorphisme de $\mathbf{G}^{F}$-variétés

$$
\mathbf{Y}_{I, v} / \mathbf{L}_{I}^{\dot{v} F} \stackrel{\sim}{\rightarrow} \mathbf{X}_{I, v}
$$

Pour finir, nous posons

$$
\overline{\mathbf{X}}_{I, v}=\left\{g \mathbf{P}_{I} \in \mathbf{G} / \mathbf{P}_{I} \mid g^{-1}{ }^{F} g \in \overline{\mathbf{P}_{I} \dot{v}{ }^{F} \mathbf{P}_{I}}\right\}
$$

et nous notons $j_{I, v}$ l'immersion ouverte canonique

$$
j_{I, v}: \mathbf{X}_{I, v} \longrightarrow \overline{\mathbf{X}}_{I, v} \text {. }
$$

C'est un morphisme de $\mathbf{G}^{F}$-variétés, où $\mathbf{G}^{F}$ agit sur $\overline{\mathbf{X}}_{I, v}$ par multiplication à gauche. De plus, $\overline{\mathbf{X}}_{I, v}$ est une variété projective. Nous noterons $\mathcal{F}_{I, v}: D^{b}\left(\Lambda \mathbf{L}_{I}^{\dot{v} F}\right) \rightarrow D_{\Lambda}^{b}\left(\mathbf{X}_{I, v}\right)$ le foncteur noté $\mathcal{F}_{\mathbf{L}_{I}^{i F}}^{\mathbf{Y}_{I, v}}$ dans le $\S 3.2$.

10.2. Transitivité et séries rationnelles. On fixe une décomposition réduite $v=$ $s_{1} \cdots s_{r}\left(s_{i} \in S\right.$ et $\left.r=l(v)\right)$ et on pose $\mathbf{v}=\left(s_{1}, \ldots, s_{r}\right)$.

Soit $\mathbf{w}=\left(w_{1}, \ldots, w_{n}\right)$ une suite finie d'éléments de $W_{I}$. On a des isomorphisme de $\mathbf{G}^{F}$-variétés-Twv $(\mathrm{cf}[\mathrm{Lu} 1$, lemme 3] et (5.5))

$$
\begin{array}{rrr}
\mathbf{Y}_{I, v} \times_{\mathbf{L}_{I} \dot{v} F} \mathbf{Y}^{\mathbf{L}_{I}, \dot{v} F}(\dot{\mathbf{w}}) \stackrel{\sim}{\rightarrow} & \mathbf{Y}^{\mathbf{G}, F}\left(\dot{w}_{1}, \ldots, \dot{w}_{n-1}, \dot{w}_{n} \dot{v}\right) \stackrel{\sim}{\rightarrow} & \mathbf{Y}^{\mathbf{G}, F}(\dot{\mathbf{w}} \dot{\mathbf{v}}) \\
\left(g \mathbf{V}_{I}\right),\left(h_{1} \mathbf{U}_{I}, \ldots, h_{n} \mathbf{U}_{I}\right) \mapsto & \left(g h_{1} \mathbf{U}, \ldots, g h_{n} \mathbf{U}\right) & \\
& \left(g_{1} \mathbf{U}, \ldots, g_{n} \mathbf{U}\right) \leftarrow & \left(g_{1} \mathbf{U}, \ldots, g_{n+r} \mathbf{U}\right) .
\end{array}
$$

Par conséquent, d'après (3.4), on a

$$
\mathcal{R}_{I, v} \circ \mathcal{R}_{\dot{\mathbf{w}}}^{\mathbf{L}_{I}, \dot{v} F} \simeq \mathcal{R}_{\dot{\mathbf{w}} \dot{\mathbf{v}}}^{\mathrm{G}, F}
$$

Si $(\mathbf{w}, \theta) \in \boldsymbol{\nabla}\left(\mathbf{T}, W_{I}, \dot{v} F\right)$, alors $(\mathbf{w v}, \theta) \in \boldsymbol{\nabla}(\mathbf{T}, W, F)$. De plus, si $(\mathbf{w}, \theta) \equiv_{W_{I}, \mathbf{v} F}$ $\left(\mathbf{w}^{\prime}, \theta^{\prime}\right)$, alors $(\mathbf{w} \mathbf{v}, \theta) \equiv_{W, F}\left(\mathbf{w}^{\prime} \mathbf{v}, \theta^{\prime}\right)$. Par suite, si $\mathcal{X}$ est une série rationnelle dans $\boldsymbol{\nabla}\left(\mathbf{T}, W_{I}, v F\right)$, il existe une unique série rationnelle $\mathcal{X}^{\mathbf{G}}$ contenant $(\mathbf{w v}, \theta)$ pour tout $(\mathbf{w}, \theta) \in \mathcal{X}$. Le théorème suivant (classique pour $\Lambda=K$ ) montre que les foncteurs de Lusztig préservent les séries rationnelles.

Théorème 10.3. Soit $\mathcal{X}$ une série rationnelle dans $\boldsymbol{\nabla}\left(\mathbf{T}, W_{I}, \dot{v} F\right)$ et soit $M$ dans $D^{b}\left(\Lambda \mathbf{L}_{I}^{\dot{v} F} e_{\mathcal{X}}^{\mathbf{L}_{I}^{\dot{v} F}}\right)$. Alors, $\mathcal{R}_{I, v}(M)$ est dans $D^{b}\left(\Lambda \mathbf{G}^{F} e_{\mathcal{X}^{\mathbf{G}}}^{\mathbf{G}^{F}}\right)$.

Démonstration. Par la formule des coefficients universels, on peut supposer, et nous le ferons, que $\Lambda$ est un corps.

Il suffit en outre de considérer le cas où $M=\Lambda \mathbf{L}_{I}^{\dot{v} F} e_{\mathcal{X}}^{\mathbf{L}_{I}^{i F}}$. Mais, d'après le théorème $\mathrm{A}, \Lambda \mathbf{L}_{I}^{\dot{v} F} e_{\mathcal{X}}^{\mathbf{L}_{I}^{\dot{v} F}}$ est dans la sous-catégorie engendrée par les complexes $\mathcal{R}_{\dot{\mathbf{w}}}^{\mathbf{L}_{I}, \dot{v} F} \Lambda \mathbf{T}^{\mathbf{w v} F} e_{\theta}$ où 
$(\mathbf{w}, \theta) \in \mathcal{X}$. Cela montre qu'il suffit de considérer le cas où $M=\mathcal{R}_{\dot{\mathbf{w}}}^{\mathbf{L}_{I}, \dot{v} F} \Lambda \mathbf{T}^{\mathbf{w v} F} e_{\theta}$ : alors, $\mathcal{R}_{I, v}(M) \simeq \mathcal{R}_{\dot{\mathbf{w}} \mathbf{v}}^{\mathbf{G}, F} \Lambda \mathbf{T}^{\mathbf{w v} F} e_{\theta}$ d'après (10.2). La conclusion provient alors du théorème A.

10.3. Equivalence de Morita. Commençons par une définition. Le couple $(\mathbf{w}, \theta) \in$ $\boldsymbol{\nabla}\left(\mathbf{T}, W_{I}, \dot{v} F\right)$ est dit $(\mathbf{G}, I)$-régulier (respectivement $(\mathbf{G}, I)$-super régulier) s'il vérifie la propriété suivante :

$$
\text { Si } \alpha \in \Phi \text { est telle que } \theta \circ N_{\mathbf{w v}}\left(\alpha^{\vee}\right)=1 \text {, alors } \alpha \in \Phi_{I} \text {. }
$$

(respectivement

$$
\text { Si } \left.x \in W \text { est tel que }{ }^{x}\left(\theta \circ N_{\mathbf{w v}}\right)=\theta \circ N_{\mathbf{w v}} \text {, alors } x \in W_{I} .\right)
$$

Lemme 10.4. Un couple $(\mathbf{G}, I)$-super régulier est $(\mathbf{G}, I)$-régulier.

Démonstration. Soient $(\mathbf{w}, \theta)$ un couple $(\mathbf{G}, I)$-super régulier et $\alpha \in \Phi$ tel que $\theta \circ N_{\mathbf{w v}}\left(\alpha^{\vee}\right)=$ 1. Pour tout $\lambda \in Y(\mathbf{T})$, on a $\left(s_{\alpha}-1\right)(\lambda) \in \mathbf{Z} \alpha^{\vee}$, donc $\theta \circ N_{\mathbf{w v}}\left(\left(s_{\alpha}-1\right)(\lambda)\right)=1$, ce qui montre que $^{s_{\alpha}}\left(\theta \circ N_{\mathbf{w v}}\right)=\theta \circ N_{\mathbf{w v}}$. D'après (SR), on a alors $s_{\alpha} \in W_{I}$, donc $\alpha \in \Phi_{I}$.

Remarque 10.5. Soient $(\mathbf{w}, \theta)$ et $\left(\mathbf{w}^{\prime}, \theta^{\prime}\right)$ deux couples de $\boldsymbol{\nabla}\left(\mathbf{T}, W_{I}, \dot{v} F\right)$ qui sont géométriquement conjugués. Alors, le couple $(\mathbf{w}, \theta)$ est $(\mathbf{G}, I)$-régulier (respectivement $(\mathbf{G}, I)$ super régulier) si et seulement si le couple $\left(\mathbf{w}^{\prime}, \theta^{\prime}\right)$ l'est. En effet, par définition de la conjugaison géométrique, $\theta \circ N_{\mathbf{w v}}$ et $\theta^{\prime} \circ N_{\mathbf{w}^{\prime} \mathbf{v}}$ sont conjugués sous $W_{I}$.

Une série rationnelle $\mathcal{X}$ dans $\boldsymbol{\nabla}\left(\mathbf{T}, W_{I}, \dot{v} F\right)$ est dite $(\mathbf{G}, I)$-régulière (respectivement $(\mathbf{G}, I)$-super régulière) si un de ses représentants l'est. Compte tenu de la remarque 10.5, $\mathcal{X}$ est $(\mathbf{G}, I)$-régulière (respectivement $(\mathbf{G}, I)$-super régulière) si et seulement si tous ses représentants le sont.

La propriété des couples réguliers qui va nous être utile par la suite est contenue dans le lemme suivant.

Lemme 10.6. Soit $(\mathbf{w}, \theta) \in \boldsymbol{\nabla}\left(\mathbf{T}, W_{I}, \dot{v} F\right)$ un couple $(\mathbf{G}, I)$-régulier avec $\mathbf{w} \in \Sigma(I \cup\{1\})$. Alors, $(\mathbf{w v})_{\theta}=\mathbf{w}_{\theta} \mathbf{v}$.

Démonstration. Notons tout d'abord que $(\mathbf{w v})_{\theta}$ est calculé dans $(\mathbf{G}, F)$, alors que $\mathbf{w}_{\theta}$ l'est dans $(\mathbf{L}, \dot{v} F)$. Remarquons ensuite que $(\mathbf{w v})_{\theta} \leq \mathbf{w}_{\theta} \mathbf{v}$. Écrivons $\mathbf{w}=\left(t_{1}, \ldots, t_{n}\right)$ et soit $i$ un entier naturel tel que $1 \leq i \leq r$. Il s'agit de démontrer que $\theta\left(N_{\mathbf{w v}}\left(\beta_{\mathbf{w v}, n+i}^{\vee}\right)\right) \neq 1$. D'après la propriété $(\mathrm{R})$, il suffit de démontrer que $\beta_{\mathbf{w v}, n+i}^{\vee} \notin \Phi_{I}$. Notons $\alpha$ la racine $\alpha_{\mathbf{w v}, n+i}=\alpha_{\mathbf{v}, i}$. Alors, $\beta_{\mathbf{w v}, n+i}^{\vee}=t_{1} \ldots t_{n} s_{1} \ldots s_{i-1}\left(\alpha^{\vee}\right)$. Mais, $t_{1} \ldots t_{n} \in W_{I}$, donc on est ramené à prouver l'assertion suivante :

$$
s_{1} \ldots s_{i-1}\left(\alpha^{\vee}\right) \notin \Phi_{I} .
$$

Puisque $\left(s_{1}, \ldots, s_{r}\right)$ est une décomposition réduite de $v$, alors $s_{1} \ldots s_{i-1}\left(\alpha^{\vee}\right)$ est une coracine positive dont l'image par $v^{-1}$ est négative. Or, par hypothèse sur $v, v^{-1}(I)=$ $\phi(I) \subset \Delta$. Donc $v^{-1}\left(\Phi_{I} \cap \Phi^{+}\right) \subset \Phi^{+}$. Cela complète la preuve du lemme 10.6.

Nous arrivons maintenant au résultat principal de ce travail : 
Théorème 10.7. Soit $\mathcal{X}$ une série rationnelle $(\mathbf{G}, I)$-régulière dans $\boldsymbol{\nabla}\left(\mathbf{T}, W_{I}, v F\right)$. Alors, le morphisme canonique

$$
\left(j_{I, v}\right)_{!} \mathcal{F}_{I, v} \Lambda \mathbf{L}_{I}^{\dot{v} F} e_{\mathcal{X}}^{\mathbf{L}_{I}^{i j}} \stackrel{\sim}{\rightarrow} \mathrm{R}\left(j_{I, v}\right)_{*} \mathcal{F}_{I, v} \Lambda \mathbf{L}_{I}^{\dot{v} F} e_{\mathcal{X}}^{\mathbf{L}_{I}^{i v F}}
$$

est un isomorphisme.

Démonstration. Notons ici $i: \overline{\mathbf{X}}_{I, v}-\mathbf{X}_{I, v} \longrightarrow \overline{\mathbf{X}}_{I, v}$ l'immersion fermée canonique. Il s'agit de démontrer que

$$
i^{*} \mathrm{R}\left(j_{I, v}\right)_{*} \mathcal{F}_{I, v}\left(\Lambda \mathbf{L}_{I}^{\dot{v} F} e_{\mathcal{X}}^{\mathbf{L}_{I}^{\dot{j} F}}\right)=0 .
$$

La formule des coefficients universels montre qu'il suffit de prouver (1) lorsque $\Lambda$ est un corps, ce que nous supposerons. D'après le théorème $\mathrm{A}$, le $\Lambda \mathbf{L}_{I}^{i j}$-module $\Lambda \mathbf{L}_{I}^{i F} e_{\mathcal{X}}^{\mathbf{L}_{I}^{i} F}$ est dans la sous-catégorie de $\Lambda \mathbf{L}_{I}^{\dot{v} F}$-parf engendrée par les complexes $\mathcal{S}_{\dot{\mathbf{w}}}^{\mathbf{L}_{I}, \dot{v} F} \Lambda \mathbf{T}^{\mathbf{w v} F} e_{\theta}$ pour $(\mathbf{w}, \theta) \in \mathcal{X}$. Par suite, il suffit de démontrer que

$$
i^{*} \mathrm{R}\left(j_{I, v}\right)_{*} \mathcal{F}_{I, v}\left(\mathcal{S}_{\dot{\mathbf{w}}}^{\mathbf{L}_{1}, \dot{v} F} \Lambda \mathrm{T}^{\mathbf{w v} F} e_{\theta}\right)=0
$$

pour tout $(\mathbf{w}, \theta) \in \mathcal{X}$ avec $\mathbf{w} \in \Sigma(I \cup\{1\})$.

Fixons un élément $(\mathbf{w}, \theta) \in \mathcal{X}$ avec $\mathbf{w} \in \Sigma(I \cup\{1\})$. On a un diagramme commutatif

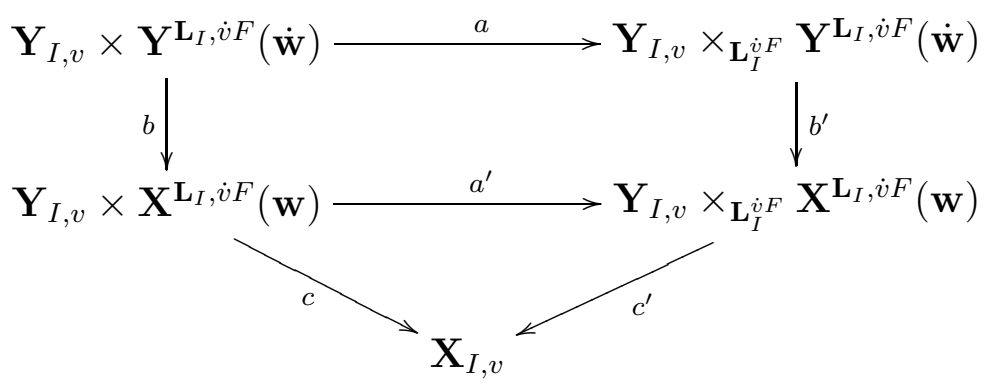

où les flèches horizontales sont les passages au quotient par $\mathbf{L}_{I}^{\dot{v} F}$, les flèches verticales les passages au quotient par $\mathbf{T}^{\mathbf{w} F} F$ et les flèches diagonales proviennent du morphisme structurel $\mathbf{X}^{\mathbf{L}_{I}, \dot{v} F}(\mathbf{w}) \rightarrow \operatorname{Spec} \mathbf{F}$.

On a des isomorphismes dans $D_{\Lambda \mathbf{T w v} F}^{b}\left(\mathbf{X}_{I, v}\right)$

$$
\begin{aligned}
\mathrm{R}\left(c^{\prime} b^{\prime}\right)_{*} \Lambda & \simeq\left(\left(\mathrm{R} c_{*}^{\prime} \mathrm{R}\left(b^{\prime} a\right)_{*} \Lambda\right) \otimes_{\Lambda \mathbf{L}_{I}^{i j}}^{\mathrm{L}} \Lambda\right. \\
& \simeq\left(\mathrm{R} c_{*} \mathrm{R} b_{*} \Lambda\right) \otimes_{\Lambda \mathbf{L}_{I}^{i j}} \Lambda,
\end{aligned}
$$

ce qui montre que

$$
\mathrm{R}\left(c^{\prime} b^{\prime}\right)_{*} \Lambda \simeq \mathcal{F}_{I, v}\left(\Lambda \mathbf{L}_{I}^{\dot{v} F}\right) \otimes_{\Lambda \mathbf{L}_{I}^{i j} F}^{\mathrm{L}} \mathrm{R} \Gamma\left(\mathbf{Y}^{\mathbf{L}_{I}, \dot{v} F}(\mathbf{w})\right)
$$

Via l'isomorphisme (10.1), le morphisme $c^{\prime}$ devient

$$
\begin{aligned}
& \tau: \quad \mathbf{X}^{\mathbf{G}, F}(\mathbf{w v}) \quad \longrightarrow \mathbf{X}_{I, v} \\
& \left(g_{1} \mathbf{B}, \ldots, g_{n} \mathbf{B}\right) \longmapsto g_{1} \mathbf{P}_{I}
\end{aligned}
$$

et après application de $-\otimes_{\Lambda \mathbf{T w v}^{\mathbf{w} F}}^{\mathrm{L}} \Lambda \mathbf{T}^{\mathbf{w v} F} e_{\theta}$, l'isomorphisme (3) devient

$$
\mathrm{R} \tau_{*} \mathcal{F}^{\mathbf{w v}}\left(\Lambda \mathbf{T}^{\mathbf{w v} F} e_{\theta}\right) \simeq \mathcal{F}_{I, v}\left(\mathcal{S}_{\dot{\mathbf{w}}}^{\mathbf{L}_{I}, \dot{v} F} \Lambda \mathbf{T}^{\mathbf{w v} F} e_{\theta}\right) .
$$


Considérons maintenant le diagramme commutatif suivant :

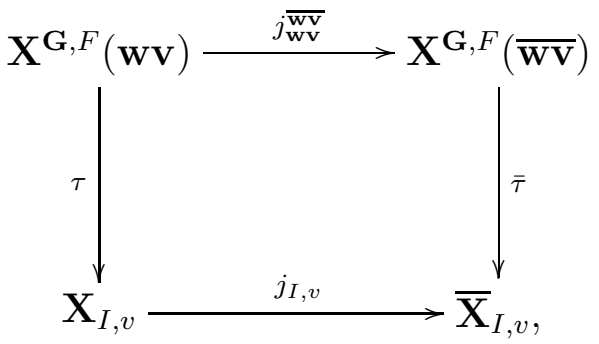

où $\bar{\tau}: \mathbf{X}^{\mathbf{G}, F}(\overline{\mathbf{w} v}) \rightarrow \overline{\mathbf{X}}_{I, v},\left(g_{1} \mathbf{B}, \ldots, g_{n} \mathbf{B}\right) \mapsto g_{1} \mathbf{P}_{I}$ est un morphisme projectif. Pour démontrer (2), il suffit, en utilisant le théorème de changement de base propre, de démontrer que la restriction du complexe $\mathrm{R}\left(j_{\mathbf{w v}}^{\overline{\mathbf{w v}}}\right)_{*} \mathcal{F}^{\mathbf{w v}}\left(\Lambda \mathbf{T}^{\mathbf{w v} F} e_{\theta}\right)$ à $\mathbf{X}^{\mathbf{G}, F}(\overline{\mathbf{w} \mathbf{v}})-\bar{\tau}^{-1}\left(\mathbf{X}_{I, v}\right)$ est nulle. Notons $i_{1}: \mathbf{X}^{\mathbf{G}, F}(\overline{\mathbf{w}})-\bar{\tau}^{-1}\left(\mathbf{X}_{I, v}\right) \hookrightarrow \mathbf{X}^{\mathbf{G}, F}(\overline{\mathbf{w v}})$ l'immersion fermée canonique. Il nous suffit de démontrer que

$$
i_{1}^{*} \mathrm{R}\left(j_{\mathbf{w v}}^{\overline{\mathbf{w v}}}\right)_{*} \mathcal{F}_{\theta}^{\mathbf{w v}}=0
$$

On a

$$
\mathbf{X}^{\mathbf{G}, F}(\overline{\mathbf{w} v})-\bar{\tau}\left(\mathbf{X}_{I, v}\right)=\coprod_{\substack{\mathbf{w}^{\prime} \leq \mathbf{w} \\ \mathbf{v}^{\prime}<\mathbf{v}}} \mathbf{X}^{\mathbf{G}, F}\left(\mathbf{w}^{\prime} \mathbf{v}^{\prime}\right) .
$$

Mais le fait que $\mathcal{X}$ soit $(\mathbf{G}, I)$-régulière implique que $(\mathbf{w} \mathbf{v})_{\theta}$ est égal à $\mathbf{w}_{\theta} \mathbf{v}$ (lemme 10.6). Le résultat découle alors du théorème 7.7 .

Remarque 10.8. Le lecteur intéressé obtiendra sans difficulté une version sans hypothèse de régularité du théorème 10.7 qui généralise le corollaire 7.6.

Corollaire 10.9. Soit $\mathcal{X}$ une série $(\mathbf{G}, I)$-régulière dans $\boldsymbol{\nabla}\left(\mathbf{T}, W_{I}, \dot{v} F\right)$. Alors, $H_{c}^{i}\left(\mathbf{X}_{I, v}, \mathcal{F}_{I, v} \Lambda \mathbf{L}_{I}^{i F} e_{\mathcal{X}}^{\mathbf{L}_{I}^{i F}}\right)$ est un $\Lambda$-module libre, nul pour $i \neq l(v)$.

Démonstration. Par la formule des coefficients universels, il suffit de démontrer l'annulation dans le cas où $\Lambda$ est un corps.

Par application du foncteur $\mathrm{R}\left(\pi_{\overline{\mathbf{X}}_{I, v}}\right)_{*}$ à l'isomorphisme du théorème 10.7, on obtient que le morphisme canonique

$$
\mathrm{R}\left(\pi_{\mathbf{X}_{I, v}}\right) ! \mathcal{F}_{I, v} \Lambda \mathbf{L}_{I}^{\dot{v} F} e_{\mathcal{X}}^{\mathbf{L}_{I}^{\dot{v} F}} \stackrel{\sim}{\rightarrow} \mathrm{R}\left(\pi_{\mathbf{X}_{I, v}}\right)_{*} \mathcal{F}_{I, v} \Lambda \mathbf{L}_{I}^{\dot{v} F} e_{\mathcal{X}}^{\mathbf{L}_{I}^{\dot{v} F}}
$$

est un isomorphisme et le lemme 12.6 (joint au lemme 3.2) fournit la conclusion.

Remarque 10.10. Si la série $\mathcal{X}$ est régulière, alors la série $\mathcal{X}^{-1}=\left\{\left(\mathbf{w}, \theta^{-1}\right) \mid(\mathbf{w}, \theta) \in \mathcal{X}\right\}$ est encore régulière. On en déduit alors, comme dans la remarque 8.11 , que l'utilisation du lemme 12.6 se fait dans le cas particulier où $M$ et $\left(M^{*}\right)^{\text {op }}$ vérifient les hypothèses.

Comme l'a montré Broué [Br, pp. 61-62], le théorème 10.7, qui est de nature géométrique, admet pour conséquence le théorème algébrique suivant (nous reprenons la preuve de $[\mathrm{Br}$, théorème 3.3]). Lorsque $\Lambda=K$ et $q$ est plus grand que le nombre de Coxeter de $\mathbf{G}$, ce 
résultat est dû à Lusztig [Lu2, Proposition 6.6]. Pour le cas général où $\Lambda=K$, seule la concentration en un degré (non déterminé) de la cohomologie était connue.

Théorème B. Soit $\mathcal{X}$ une série $(\mathbf{G}, I)$-super régulière dans $\boldsymbol{\nabla}\left(\mathbf{T}, W_{I}, \dot{v} F\right)$. Alors, $\mathcal{R}_{I, v}$ induit une équivalence de Morita entre les $\Lambda$-algèbres $\Lambda \mathbf{L}_{I}^{\dot{v} F} e_{\mathcal{X}}^{\mathbf{L}_{I}^{\dot{v} F}}$ et $\Lambda \mathbf{G}^{F} e_{\mathcal{X}^{\mathbf{G}}}^{\mathbf{G}^{F}}$.

Plus précisément, le complexe $\mathcal{R}_{I, v} \Lambda \mathbf{L}_{I}^{\dot{v} F} e_{\mathcal{X}}^{\mathbf{L}_{I}^{i F}} d e\left(\Lambda \mathbf{G}^{F}, \Lambda \mathbf{L}_{I}^{\dot{v} F} e_{\mathcal{X}}^{\mathbf{L}_{I}^{i j F}}\right)$-bimodules n'a de la cohomologie qu'en degré $r=l(v)$ et son $r$-ième bimodule de cohomologie induit l'équivalence de Morita décrite.

Démonstration. Pour montrer l'équivalence de Morita, il suffit de considérer le cas où $\Lambda=$ $R$. D'après le lemme 10.4 et le corollaire 10.9 , le bimodule $M=H_{c}^{r}\left(\mathbf{X}_{I, v}, \mathcal{F}_{I, v} R \mathbf{L}_{I}^{\dot{v} F} e_{\mathcal{X}}^{\mathbf{L}_{I}^{\dot{v} F}}\right)$ est le seul groupe de cohomologie non nul du complexe parfait de $R \mathbf{G}^{F}$-modules $\mathcal{R}_{I, v} R \mathbf{L}_{I}^{\dot{v} F} e_{\mathcal{X}}^{\mathbf{L}_{I}^{i j}}$, donc il est projectif comme $R \mathbf{G}^{F}$-module. De même, il est projectif comme $R \mathbf{L}_{I}^{\dot{v} F} e_{\mathcal{X}}^{\mathbf{L}_{I}^{\dot{v} F}}-$ module à droite.

Soit $M_{K}=K \otimes_{R} M$. D'après [Lu1, théorème 8], la $(\mathbf{G}, I)$-super régularité de $\mathcal{X}$ montre que

$$
M_{K}^{*} \otimes_{K \mathbf{G}^{F}} M_{K} \simeq K \mathbf{L}_{I}^{\dot{v} F} e_{\mathcal{X}}^{\mathbf{L}_{I}^{\dot{v} F}}
$$

Puisque $K \mathbf{G}^{F} e_{\mathcal{X}^{\mathbf{G}}}^{\mathbf{G}^{F}}$ est facteur direct du $\left(K \mathbf{G}^{F} e_{\mathcal{X}^{\mathbf{G}}}^{\mathbf{G}^{F}}, K \mathbf{G}^{F} e_{\mathcal{X}^{\mathbf{G}}}^{\mathbf{G}^{F}}\right)$-bimodule $M_{K} \otimes_{K \mathbf{L}_{I}^{i F}} M_{K}^{*}$, on en déduit que $M_{K}$ induit une équivalence de Morita entre $K \mathbf{L}_{I}^{i F} e_{\mathcal{X}}^{\mathbf{L}_{I}^{i F}}$ et $K \mathbf{G}^{F} e_{\mathcal{X}^{\mathbf{G}}}^{\mathbf{G}^{F}}$. L'équivalence de Morita sur $R$ résulte maintenant de [Br, théorème 2.4].

Remarque 10.11. Le théorème précédent montre la coïncidence des matrices de décomposition. Cette propriété avait été conjecturée par Hiß [Hi, p.342].

Remarque 10.12. D'après [Lu1, proposition 12], on a $\operatorname{dim} M_{K} \otimes_{K \mathbf{L}_{I}^{\dot{v} F}} V=n \operatorname{dim} V$, pour tout $K \mathbf{L}_{I}^{\dot{v} F} e_{\mathcal{X}^{\prime}}^{\mathbf{L}_{I}^{\dot{v} F}}$-module simple $V$, où $n=\frac{\left|\mathbf{G}^{F}\right|_{p^{\prime}}}{\left|\mathbf{L}_{I}^{v F}\right|_{p^{\prime}}}$. On déduit alors du théorème $\mathrm{B}$ que $\Lambda \mathbf{G}^{F} e_{\mathcal{X}^{\mathbf{G}}}^{\mathbf{G}^{F}}$ est isomorphe à une algèbre de matrices de dimension $n^{2} \operatorname{sur} \Lambda \mathbf{L}_{I}^{\dot{v} F} e_{\mathcal{X}^{\prime}}^{\mathbf{v}^{\dot{v} F}}$.

\section{Changement De POint De Vue}

Nous avons pris le parti, pour la commodité des preuves, de travailler systématiquement avec des sous-groupes de Levi standards, quitte à modifier l'isogénie $F$. Le but de cette partie est de traduire les résultats principaux qui précèdent en ne s'intéressant qu'aux sousgroupes de Levi et aux tores maximaux $F$-stables. Ceci permet d'alléger les notations et certains lecteurs y retrouveront un cadre plus familier.

Nous nous bornerons à énoncer les analogues des théorèmes A, 10.3, 10.7 et B.

11.1. Notations. Soit $\mathbf{P}$ un sous-groupe parabolique de $\mathbf{G}$ de radical unipotent $\mathbf{V}$, avec un complément de Levi $F$-stable L. Nous posons

et

$$
\mathbf{Y}_{\mathbf{V}}^{\mathbf{G}}=\left\{g \mathbf{V} \in \mathbf{G} / \mathbf{V} \mid g^{-1} F(g) \in \mathbf{V} \cdot{ }^{F} \mathbf{V}\right\}
$$

$$
\mathbf{X}_{\mathbf{P}}^{\mathbf{G}}=\left\{g \mathbf{P} \in \mathbf{G} / \mathbf{P} \mid g^{-1} F(g) \in \mathbf{P} \cdot{ }^{F} \mathbf{P}\right\} \text {. }
$$


Alors, $\mathbf{Y}_{\mathbf{V}}^{\mathbf{G}}$ est une $\mathbf{G}^{F}$-variété- $\mathbf{L}^{F}$ (pour les actions par multiplication à gauche et à droite), $\mathbf{X}_{\mathbf{P}}^{\mathbf{G}}$ est une $\mathbf{G}^{F}$-variété et le morphisme étale $\pi_{\mathbf{V}}: \mathbf{Y}_{\mathbf{V}}^{\mathbf{G}} \rightarrow \mathbf{X}_{\mathbf{P}}^{\mathbf{G}}$ induit un isomorphisme de variétés $\mathbf{Y}_{\mathbf{V}}^{\mathbf{G}} / \mathbf{L}^{F} \simeq \mathbf{X}_{\mathbf{P}}^{\mathbf{G}}$ (cf (5.3) et $\left.§ 11.2\right)$. Nous définissons alors les foncteurs

$$
\mathcal{R}_{\mathbf{L} \subset \mathbf{P}}^{\mathbf{G}}: D^{b}\left(\Lambda \mathbf{L}^{F}\right) \longrightarrow D^{b}\left(\Lambda \mathbf{G}^{F}\right)
$$

et

$$
\mathcal{S}_{\mathbf{L} \subset \mathbf{P}}^{\mathbf{G}}: D^{b}\left(\Lambda \mathbf{L}^{F}\right) \longrightarrow D^{b}\left(\Lambda \mathbf{G}^{F}\right)
$$

comme au $\S 3.2$.

Pour finir, posons

$$
\overline{\mathbf{X}}_{\mathbf{P}}^{\mathbf{G}}=\left\{g \mathbf{P} \in \mathbf{G} / \mathbf{P} \mid g^{-1} F(g) \in \overline{\mathbf{P} \cdot F \mathbf{P}}\right\}
$$

et notons $j_{\mathbf{P}}^{\mathbf{G}}: \mathbf{X}_{\mathbf{P}}^{\mathbf{G}} \hookrightarrow \overline{\mathbf{X}}_{\mathbf{P}}^{\mathbf{G}}$ l'immersion ouverte canonique.

11.2. Rapport avec les groupes $\mathbf{L}_{I}^{\dot{v} F}$. Nous expliquons ici le lien entre les variétés $\mathbf{Y}_{\mathbf{V}}^{\mathbf{G}}$ et les variétés $\mathbf{Y}_{I, v}$ définies précédemment. Soit $I$ l'unique partie de $\Delta$ telle que $\mathbf{P}$ soit conjugué à $\mathbf{P}_{I}$. Il existe alors un élément $x_{1} \in \mathbf{G}$ tel que ${ }^{x_{1}}\left(\mathbf{L}_{I}, \mathbf{P}_{I}\right)=(\mathbf{L}, \mathbf{P})$. Posons $v_{1}=x_{1}^{-1} F\left(x_{1}\right)$. Puisque $\mathbf{L}$ est $F$-stable, on a ${ }^{v_{1} F} \mathbf{L}_{I}=\mathbf{L}_{I}$. Par conséquent, il existe $a \in \mathbf{L}_{I}$ tel que ${ }^{a v_{1} F}\left(\mathbf{T}, \mathbf{B}_{I}\right)=\left(\mathbf{T}, \mathbf{B}_{I}\right)$. Notons $v$ l'image de $a v_{1} \in N_{\mathbf{G}}(\mathbf{T})$ dans $W$. Nous choisissons $a$ de sorte que $a v_{1}=\dot{v}$. Par le théorème de Lang, on trouve $b \in \mathbf{L}_{I}$ tel que $b^{-1} v_{1} F b=a$.

Posons maintenant $x=x_{1} b$. Alors $x^{-1} F(x)=b^{-1} v_{1} F(b)=a v_{1}=\dot{v}$. De plus $\dot{v} F$ stabilise $\mathbf{B}_{I}$, donc $v \phi(I)=I$. D'autre part, ${ }^{x}\left(\mathbf{L}_{I}, \mathbf{P}_{I}\right)=(\mathbf{L}, \mathbf{P})$ et l'application

$$
\begin{aligned}
& \mathbf{L}_{I} \longrightarrow \quad \mathbf{L} \\
& g \longmapsto x g x^{-1}
\end{aligned}
$$

induit un isomorphisme $\mathbf{L}_{I}^{\dot{v} F} \stackrel{\sim}{\rightarrow} \mathbf{L}^{F}$, que nous noterons aussi par la lettre $x$. De plus, ${ }^{x} \mathbf{V}_{I}=\mathbf{V}$.

Les applications

$$
\begin{aligned}
& \mathbf{Y}_{\mathbf{V}}^{\mathbf{G}} \longrightarrow \mathbf{Y}_{I, v} \\
& g \mathbf{V} \longmapsto g x \mathbf{V}_{I}, \\
& \mathbf{X}_{\mathbf{P}}^{\mathbf{G}} \longrightarrow \mathbf{X}_{I, v} \\
& g \mathbf{P} \longmapsto g x \mathbf{P}_{I} \\
& \\
& \overline{\mathbf{X}}_{\mathbf{P}}^{\mathbf{G}} \longrightarrow \overline{\mathbf{X}}_{I, v} \\
& g \mathbf{P} \longmapsto g x \mathbf{P}_{I}
\end{aligned}
$$

et

sont des isomorphismes, de $\mathbf{G}^{F}$-variétés- $\mathbf{L}^{F}$ pour le premier, de $\mathbf{G}^{F}$-variétés pour le second et le troisième. Ici, $\mathbf{Y}_{I, v}$ est vue comme une variété- $\mathbf{L}^{F}$ via l'isomorphisme $x^{-1}$. De plus, 
le diagramme correspondant

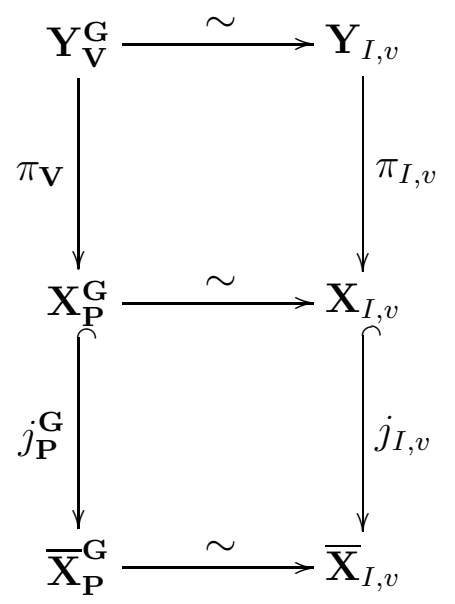

est commutatif.

Ces isomorphismes permettent de faire le lien entre tous les objets définis précédemment. Par exemple, on a un diagramme commutatif

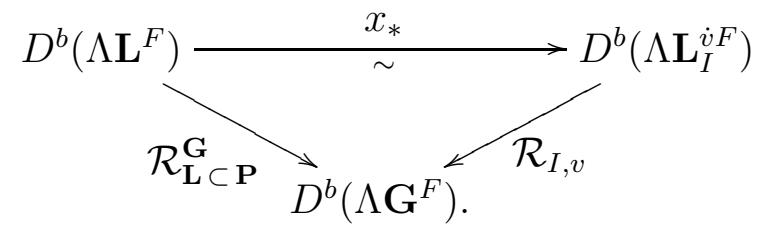

qui montre que l'on peut jongler entre les divers foncteurs de Lusztig.

Remarque 11.2. Il est facile de montrer que, si $\left(I^{\prime}, v^{\prime}\right)$ est un autre couple vérifiant les propriétés suivantes :

(1) $I^{\prime} \subset \Delta, v^{\prime} \in W$,

(2) $v^{\prime} \phi\left(I^{\prime}\right)=I^{\prime}$,

(3) il existe $x^{\prime} \in \mathbf{G}$ tel que ${ }^{x^{\prime}}\left(\mathbf{L}_{I^{\prime}}, \mathbf{P}_{I^{\prime}}\right)=(\mathbf{L}, \mathbf{P})$ et $x^{\prime-1} F x^{\prime}=\dot{v}^{\prime}$, alors $(I, v)=\left(I^{\prime}, v^{\prime}\right)$. On dira que le couple $(I, v)$ est associé au couple $(\mathbf{L}, \mathbf{P})$.

11.3. Séries rationnelles. Notons $\boldsymbol{\nabla}(\mathbf{G}, F)$ l'ensemble des triplets $\left(\mathbf{T}^{\prime}, \mathbf{B}^{\prime}, \theta^{\prime}\right)$, où $\mathbf{T}^{\prime}$ est un tore maximal $F$-stable de $\mathbf{G}, \mathbf{B}^{\prime}$ est un sous-groupe de Borel de $\mathbf{G}$ contenant $\mathbf{T}^{\prime}$ et $\theta^{\prime}: \mathbf{T}^{\prime F} \rightarrow \Lambda^{\times}$un caractère linéaire d'ordre inversible dans $\Lambda$. Le choix de $\imath$ et $\jmath$ fournit une partition de $\boldsymbol{\nabla}(\mathbf{G}, F)$ en séries rationnelles, i.e., selon les classes de $\mathbf{G}^{* F^{*}}$ conjugaison d'éléments de $\mathbf{G}_{\mathrm{sem}, \Lambda}^{* F^{*}}$ (on note $\mathbf{G}_{\mathrm{sem}, \Lambda}^{* F^{*}}$ l'ensemble des éléments semi-simples de $\mathbf{G}^{* F^{*}}$ d'ordre inversible dans $\left.\Lambda\right)$. Nous noterons $\boldsymbol{\nabla}\left(\mathbf{G}, F,(s)_{\mathbf{G}^{*} F^{*}}\right)$ la série rationnelle associée à $s \in \mathbf{G}_{\text {sem, },}^{* F^{*}}$.

Si $\left(\mathbf{T}^{\prime}, \mathbf{B}^{\prime}, \theta^{\prime}\right) \in \boldsymbol{\nabla}(\mathbf{L}, F)$, alors il existe un unique élément $w \in W_{I}$ tel que $(\emptyset, w v)$ soit associé à $\left(\mathbf{T}^{\prime}, \mathbf{B}^{\prime} \mathbf{V}\right)$. Il existe un élément $y \in \mathbf{G}$ tel que ${ }^{y}(\mathbf{T}, \mathbf{B})=\left(\mathbf{T}^{\prime}, \mathbf{B}^{\prime} \mathbf{V}\right)$ et $y^{-1}{ }^{F} y=\dot{w} \dot{v}$ et, comme dans le paragraphe précédent, $y$ induit un isomorphisme $\mathbf{T}^{\prime F} \stackrel{\sim}{\rightarrow} \mathbf{T}^{w v F}$. On note $\theta$ le caractère linéaire de $\mathbf{T}^{w v F}$ correspondant à $\theta^{\prime}$ via $y$. Alors, $((w), \theta)$ est dans $\boldsymbol{\nabla}\left(\mathbf{T}, W_{I}, \dot{v} F\right)$ et il est défini de manière unique par le triplet $\left(\mathbf{T}^{\prime}, \mathbf{B}^{\prime}, \theta^{\prime}\right)$. 
Nous avons donc construit une application

$$
\psi_{\mathbf{L} \subset \mathbf{P}}: \nabla(\mathbf{L}, F) \longrightarrow \nabla\left(\mathbf{T}, W_{I}, v F\right) .
$$

Lorsque $\mathbf{L}=\mathbf{G}$ (auquel cas $I=\Delta$ et $v=1$ ), nous la noterons simplement $\psi$. Cette application conserve les séries rationnelles. Mieux, si $\mathcal{X}$ est une série rationnelle dans $\boldsymbol{\nabla}(\mathbf{T}, W, F)$ et si $((\dot{w}), \theta) \in \mathcal{X}$, alors il existe $\left(\mathbf{T}^{\prime}, \mathbf{B}^{\prime}, \theta^{\prime}\right) \in \boldsymbol{\nabla}(\mathbf{G}, F)$ tel que $\psi\left(\mathbf{T}^{\prime}, \mathbf{B}^{\prime}, \theta^{\prime}\right)=$ $((\dot{w}), \theta)$. On a alors

$$
\mathcal{R}_{\mathbf{T}^{\prime} \subset \mathbf{B}^{\prime}}^{\mathbf{G}^{\prime}} \Lambda \mathbf{T}^{\prime F} e_{\theta^{\prime}} \stackrel{\sim}{\rightarrow} \mathcal{R}_{\dot{w}} \Lambda \mathbf{T}^{w F} e_{\theta}
$$

Cette discussion montre que le théorème $\mathrm{A}$ devient :

Théorème A'. Pour $(s)_{\mathbf{G}^{* F^{*}}} \in \mathbf{G}_{\mathrm{sem}, \Lambda}^{* F^{*}} / \sim$, il existe un idempotent central $e_{s}=e_{s}^{\mathbf{G}^{F}}=e_{\Lambda, s}^{\mathbf{G}^{F}}$ de $\Lambda \mathbf{G}^{F}$ tel que la famille $\left.\left(\mathcal{R}_{\mathbf{T}^{\prime} \subset \mathbf{B}^{\prime}}^{\mathbf{G}} \Lambda \mathbf{T}^{\prime F} e_{\theta}\right)_{\left(\mathbf{T}^{\prime}, \mathbf{B}^{\prime}, \theta\right) \in \boldsymbol{\nabla}(\mathbf{G}, F,(s)} \mathbf{G}^{* F^{*}}\right)$ (respectivement

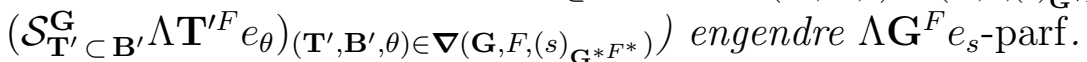

On a une décomposition de 1 en somme d'idempotents centraux deux à deux orthogonaux

$$
1=\sum_{(s)_{\mathbf{G}^{*} F^{*}} \in \mathbf{G}_{\mathrm{sem}, \Lambda}^{* F^{*}} / \sim}^{\perp} e_{s} .
$$

Remarque 11.3. On a $\mathbf{G}_{\mathrm{sem}, R}^{* F^{*}}=\mathbf{G}_{\mathrm{sem}, k}^{* F^{*}}$. Pour $s \in \mathbf{G}_{\mathrm{sem}, R}^{* F^{*}}$, la remarque 9.3 montre que

$$
e_{R, s}=\sum_{\substack{(t)_{\mathbf{G}^{*} F^{*} \in \mathbf{G}_{\text {sem }}^{* F^{*}} / \sim} \\\left(t_{\ell^{\prime}}\right)_{\mathbf{G}^{*} F^{*}=(s)} \mathbf{G}^{* F^{*}}}} e_{K, t} .
$$

De plus, si $(t)_{\mathbf{G}^{* F^{*}}} \in \mathbf{G}_{\mathrm{sem}}^{* F^{*}} / \sim$, alors

$$
e_{K, t}=\sum_{\chi \in \mathcal{E}\left(\mathbf{G}^{F},(t)\right.} e_{\mathbf{G}^{*} F^{*}},
$$

où $\mathcal{E}\left(\mathbf{G}^{F},(t)_{\mathbf{G}^{* F^{*}}}\right)$ est la série de Lusztig rationnelle associée à $(t)_{\mathbf{G}^{* F^{*}}}$.

Le théorème 10.3 devient quant à lui :

Théorème 11.4. Soit $s \in \mathbf{L}_{\mathrm{sem}, \Lambda}^{* F^{*}}$ et soit $M \in D^{b}\left(\Lambda \mathbf{L}^{F} e_{s}^{\mathbf{L}^{F}}\right)$. Alors, $\mathcal{R}_{\mathbf{L} \subset \mathbf{P}}^{\mathbf{G}}(M) \in D^{b}\left(\Lambda \mathbf{G}^{F} e_{s}^{\mathbf{G}^{F}}\right)$.

11.4. Equivalence de Morita. Pour finir la traduction et obtenir des résultats équivalents aux théorèmes 10.7 et $\mathrm{B}$, nous devons voir ce que deviennent les définitions de séries régulières et super-régulières à travers l'application $\psi$ définie au paragraphe précédent.

Fixons un élément semi-simple $s \in \mathbf{G}_{\text {sem, }, \Lambda}^{* F^{*}}$ et un triplet $\left(\mathbf{T}^{\prime}, \mathbf{B}^{\prime}, \theta^{\prime}\right) \in \boldsymbol{\nabla}\left(\mathbf{G}, F,(s)_{\mathbf{G}^{*} F^{*}}\right)$. Notons $\mathbf{T}^{*}$ un tore maximal $F^{*}$-stable de $\mathbf{G}^{*}$ dual de $\mathbf{T}^{\prime}$.

Puisque $\left(\mathbf{T}^{\prime}, \mathbf{B}^{\prime}, \theta^{\prime}\right) \in \boldsymbol{\nabla}\left(\mathbf{G}, F,(s)_{\mathbf{G}^{*} F^{*}}\right)$, on peut supposer que $s \in \mathbf{T}^{* F^{*}}$. Alors il existe $\lambda \in Y\left(\mathbf{T}^{*}\right)=X\left(\mathbf{T}^{\prime}\right)$ tel que $s=N_{F^{* d} / F^{*}}(\lambda(\zeta))$. On a alors, pour tout $\mu \in Y\left(\mathbf{T}^{\prime}\right)=$ $X\left(\mathbf{T}^{*}\right)$,

$$
\theta^{\prime}\left(N_{F^{d} / F}(\mu(\zeta))\right)=\kappa\left(\zeta^{\left\langle\lambda, N_{F^{d} / F}(\mu)\right\rangle}\right)=\kappa(\mu(s))
$$


Fixons maintenant un sous-groupe de Levi $F^{*}$-stable $\mathbf{L}^{*}$ d'un sous-groupe parabolique de $\mathbf{G}^{*}$ dual de $\mathbf{L}$ et supposons que $s \in \mathbf{L}_{\text {sem }, \Lambda}^{* F^{*}}$. La série $\boldsymbol{\nabla}\left(\mathbf{L}, F,(s)_{\mathbf{L}^{* F^{*}}}\right)$ est dite $(\mathbf{G}, \mathbf{L})$ régulière (respectivement $(\mathbf{G}, \mathbf{L})$-super-régulière) si $\psi_{\mathbf{L} \subset \mathbf{P}}\left(\boldsymbol{\nabla}\left(\mathbf{L}, F,(s)_{\mathbf{L}^{* F^{*}}}\right)\right)$ est contenue dans une série $(\mathbf{G}, I)$-régulière (respectivement $(\mathbf{G}, I)$-super-régulière) de $\boldsymbol{\nabla}\left(\mathbf{T}, W_{I}, \dot{v} F\right)$.

Lemme 11.6. Avec les notations ci-dessus, $\boldsymbol{\nabla}\left(\mathbf{L}, F,(s)_{\mathbf{L}^{* F^{*}}}\right)$ est $(\mathbf{G}, \mathbf{L})$-régulière (respectivement $(\mathbf{G}, \mathbf{L})$-super-régulière) si et seulement si $C_{\mathbf{G}^{*}}^{\circ}(s) \subset \mathbf{L}^{*}$ (respectivement si et seulement si $\left.C_{\mathbf{G}^{*}}(s) \subset \mathbf{L}^{*}\right)$.

Démonstration. L'égalité 11.5 montre qu'une coracine de $\alpha^{\vee}$ de $\mathbf{G}$ relative à $\mathbf{T}^{\prime}$ vérifie $\theta^{\prime}\left(N_{F^{d} / F}\left(\alpha^{\vee}(\zeta)\right)\right)=1$ si et seulement si elle vérifie $\alpha^{\vee}(s)=1$ (ici, $Y\left(\mathbf{T}^{\prime}\right)$ a été identifié avec $\left.X\left(\mathbf{T}^{*}\right)\right)$. Donc $\boldsymbol{\nabla}\left(\mathbf{L}, F,(s)_{\mathbf{L}^{* F^{*}}}\right)$ est $(\mathbf{G}, \mathbf{L})$-régulière si et seulement si $C_{\mathbf{G}^{*}}^{\circ}(s) \subset \mathbf{L}^{*}$.

D'autre part, la même égalité 11.5 montre qu'un élément $w \in N_{\mathbf{G}}\left(\mathbf{T}^{\prime}\right) / \mathbf{T}^{\prime}$ stabilise le caractère $\theta^{\prime}$ si et seulement si son correspondant $w^{*}$ dans $N_{\mathbf{G}^{*}}\left(\mathbf{T}^{* *}\right) / \mathbf{T}^{* *}$ stabilise $s$. Donc $\boldsymbol{\nabla}\left(\mathbf{L}, F,(s)_{\mathbf{L}^{* F^{*}}}\right)$ est $(\mathbf{G}, \mathbf{L})$-super-régulière si et seulement si $C_{\mathbf{G}^{*}}(s) \subset \mathbf{L}^{*}$.

Avant d'énoncer un équivalent du théorème 10.7, nous avons besoin de quelques notations. Si $s \in \mathbf{L}_{\mathrm{sem}, \Lambda}^{* F^{*}}$, nous noterons $\mathcal{F}_{s}^{\mathbf{L}}$ le système local sur la variété $\mathbf{X}_{\mathbf{P}}^{\mathbf{G}}$ associé au $\Lambda \mathbf{L}^{F}$-module $\Lambda \mathbf{L}^{F} e_{s}^{\mathbf{L}^{F}}$. Il résulte alors immédiatement du lemme 11.6 que le théorème 10.7 est équivalent au théorème suivant :

Théorème 11.7. Soit $s \in \mathbf{L}_{\mathrm{sem}, \Lambda}^{* F^{*}}$. Si $C_{\mathbf{G}^{*}}^{\circ}(s) \subset \mathbf{L}^{*}$, alors le morphisme canonique de complexes $\left(j_{\mathbf{P}}^{\mathbf{G}}\right) ! \mathcal{F}_{s}^{\mathbf{L}} \stackrel{\sim}{\rightarrow} \mathrm{R}\left(j_{\mathbf{P}}^{\mathbf{G}}\right)_{*} \mathcal{F}_{s}^{\mathbf{L}}$ est un isomorphisme.

De même, d'après le lemme 11.6, le théorème B est équivalent au théorème suivant, conjecturé par Broué [Br, p.61] :

Théorème B'. Soit $s \in \mathbf{L}_{\mathrm{sem}, \Lambda}^{* F^{*}}$ tel que $C_{\mathbf{G}^{*}}(s) \subset \mathbf{L}^{*}$. Alors, le foncteur $\mathcal{R}_{\mathbf{L} \subset \mathbf{P}}^{\mathbf{G}}$ induit une équivalence de Morita entre les $\Lambda$-algèbres $\Lambda \mathbf{L}^{F} e_{s}^{\mathbf{L}^{F}}$ et $\Lambda \mathbf{G}^{F} e_{s}^{\mathbf{G}^{F}}$.

Plus précisément, le complexe $\mathcal{R}_{\mathbf{L} \subset \mathbf{P}^{\mathbf{G}}} \Lambda \mathbf{L}^{F} e_{s}^{\mathbf{L}^{F}} n^{\prime}$ 'a de la cohomologie qu'en degré $r=$ $\operatorname{dim} \mathbf{V}-\operatorname{dim} \mathbf{V} \cap{ }^{F} \mathbf{V}$ et son r-ième bimodule de cohomologie induit l'équivalence de Morita décrite.

11.5. Décomposition de Jordan. Rappelons tout d'abord que si $s \in \mathbf{G}_{\mathrm{sem}, \Lambda}^{* F^{*}}$ est central, alors il existe un caractère linéaire $\hat{s}$ de $\mathbf{G}^{F}$ tel que l'automorphisme d'algèbre de $\Lambda \mathbf{G}^{F}$ donné par $g \mapsto \hat{s}(g) g$ se restreint en un isomorphisme

$(\#)_{\mathbf{G}}$

$$
\Lambda \mathbf{G}^{F} e_{s}^{\mathbf{G}^{F}} \stackrel{\sim}{\rightarrow} \Lambda \mathbf{G}^{F} e_{1}^{\mathbf{G}^{F}} .
$$

Revenons au cas d'un élément général $s \in \mathbf{G}_{\mathrm{sem}, \Lambda}^{* F^{*}}$. On note $\mathbf{L}^{*}(s)$ le sous-groupe de Levi minimal de $\mathbf{G}^{*}$ contenant $C_{\mathbf{G}^{*}}(s)$ (il est $F^{*}$-stable). On note $\mathbf{L}(s)$ un sous-groupe de Levi $F$-stable de $\mathbf{G}$ dual de $\mathbf{L}^{*}(s)$. Le théorème $\mathrm{B}^{\prime}$ fournit une équivalence de Morita entre $\Lambda \mathbf{L}(s)^{F} e_{s}^{\mathbf{L}(s)^{F}}$ et $\Lambda \mathbf{G}^{F} e_{s}^{\mathbf{G}^{F}}$. Ceci ramène l'étude des blocs, à équivalence de Morita près, à l'étude de blocs associés à un élément semi-simple quasi-isolé (ie, à un élément $s \in \mathbf{G}_{\text {sem }}^{*}$ tel que $C_{\mathbf{G}^{*}}(s)$ n'est contenu dans aucun sous-groupe de Levi strict). Pour un groupe à centre connexe, un élément semi-simple quasi-isolé du dual est un élément isolé (ie, un élément $s \in \mathbf{G}_{\text {sem }}^{*}$ tel que $C_{\mathbf{G}^{*}}^{\circ}(s)$ n'est contenu dans aucun sous-groupe de Levi strict). 
Théorème 11.8. Si $C_{\mathbf{G}^{*}}\left(\right.$ s) est un sous-groupe de Levi de $\mathbf{G}^{*}$, alors pour tout sous-groupe parabolique $\mathbf{P}(s)$ de $\mathbf{G}$ de complément de Levi $\mathbf{L}(s)$, le foncteur $\mathcal{R}_{\mathbf{L}(s) \subset \mathbf{P}(s)}^{\mathbf{G}}\left(\hat{s} \otimes_{\Lambda}-\right)$ induit une équivalence de Morita entre $\Lambda \mathbf{L}(s)^{F} e_{1}^{\mathbf{L}(s)^{F}}$ et $\Lambda \mathbf{G}^{F} e_{s}^{\mathbf{G}^{F}}$.

Deux raisons font que $C_{\mathbf{G}^{*}}(s)$ n'est pas nécessairement un sous-groupe de Levi de $\mathbf{G}^{*}$. D'abord, $C_{\mathbf{G}^{*}}(s)$ peut ne pas être connexe (cela ne peut être arriver que si $Z(\mathbf{G})$ n'est pas connexe). Ensuite, le groupe $C_{\mathbf{G}^{*}}^{\circ}(s)$ peut aussi ne pas être un sous-groupe de Levi de $\mathbf{G}^{*}$ (cela ne peut arriver que si l'ordre de $s$ est divisible par un nombre premier mauvais pour $\mathbf{G})$.

Plus précisément, soit $\pi_{1}$ (respectivement $\pi_{2}$ ) l'ensemble des nombres premiers qui divisent $\left|Z(\mathbf{G}) / Z(\mathbf{G})^{\circ}\right|$ (respectivement qui sont mauvais pour $\mathbf{G}$ ). On pose $\pi=\pi_{1} \cup \pi_{2}$. Si $s$ est un $\pi^{\prime}$-élément, alors $C_{\mathbf{G}^{*}}(s)$ est un sous-groupe de Levi de $\mathbf{G}^{*}$ et le théorème 11.8 s'applique.

Pour un groupe de type $A$ à centre connexe (par exemple un groupe linéaire ou unitaire), alors $\pi=\emptyset$ : on a démontré la décomposition de Jordan des blocs.

Pour un groupe de type $B, C$ ou $D$, alors $\pi=\{2\}$. On a en particulier une décomposition de Jordan des blocs pour $\ell=2$ et $\Lambda=R / \mathfrak{l}^{n}$.

Remarque 11.9. On ne dispose pas de construction (géométrique) d'un foncteur qui pourrait induire une équivalence fournissant une décomposition de Jordan générale.

\section{Appendice : QUELQUES LEMMES GÉOMÉtRIQUeS}

Nous rassemblons ici plusieurs résultats géométriques utiles dans le reste du texte. Plus précisément, nous donnons quelques propriétés des foncteurs entre catégories de $\Lambda$ faisceaux constructibles (images directes ou inverses, à support propre ou non). La plupart de ces propriétés est bien connue (formules d'adjonction, de Künneth, diviseurs lisses à croisements normaux). Nous avons tout de même inclus une preuve lorsque nous n'avons pas trouvé de référence satisfaisante. Les lemmes 12.3 et 12.6, qui semblent être nouveaux, sont une variation sur un thème classique.

12.1. Autour de la formule de Künneth. Soit $f: \mathbf{X}_{1} \rightarrow \mathbf{X}_{2}$ un morphisme de variétés algébriques, soit $A$ une $\Lambda$-algèbre finie et soit $\mathcal{X}_{1}$ (respectivement $\mathcal{X}_{2}$ ) un objet de $D_{A^{\text {op }}}^{b}\left(\mathbf{X}_{1}\right)$ (respectivement de $D_{A}^{b}\left(\mathbf{X}_{2}\right)$ ). Le lemme suivant est un cas particulier de la formule de Künneth (cf par exemple [Mi, chapitre VI, remarque 8.14]).

Lemme 12.1. Le morphisme canonique $\left(\mathrm{R} f_{!} \mathcal{X}_{1}\right) \otimes_{A}^{\mathrm{L}} \mathcal{X}_{2} \stackrel{\sim}{\rightarrow} \mathrm{R} f_{!}\left(\mathcal{X}_{1} \otimes_{A}^{\mathrm{L}} f^{*} \mathcal{X}_{2}\right)$ est un quasiisomorphisme bifonctoriel.

Le lemme qui suit est lui aussi classique.

Lemme 12.2. Si $\mathcal{X}_{2}$ est à cohomologie localement constante, alors le morphisme canonique $\left(\mathrm{R} f_{*} \mathcal{X}_{1}\right) \otimes_{A}^{\mathrm{L}} \mathcal{X}_{2} \stackrel{\sim}{\rightarrow} \mathrm{R} f_{*}\left(\mathcal{X}_{1} \otimes_{A}^{\mathrm{L}} f^{*} \mathcal{X}_{2}\right)$ est un quasi-isomorphisme bifonctoriel.

Démonstration. Il suffit de démontrer le lemme pour $\Lambda=R / \mathfrak{l}^{n}$ avec $n>0$. Il suffit aussi de traiter le cas où $\mathcal{X}_{2}$ est un faisceau. Le faisceau $\mathcal{X}_{2}$ est localement constant et l'isomorphisme est de nature locale, donc on peut supposer, en utilisant le théorème de 
changement de base lisse [Mi, chapitre VI, théorème VI.4.1] pour le morphisme $f$, qu'il est constant. Dans ce cas, le lemme 12.2 est clair.

12.2. Quasi-affinité. Soit $\mathbf{X}$ une variété et $\mathcal{F}$ un faisceau constructible sur $X$. Soit $D$ le foncteur de dualité de Verdier, $D=\operatorname{RHom} \bullet\left(-, \pi_{\mathbf{X}}^{!} \Lambda\right)$.

Considérons le morphisme canonique de complexes $\operatorname{can}_{\mathcal{F}}: \mathrm{R}\left(\pi_{\mathbf{X}}\right)_{!} \mathcal{F} \rightarrow \mathrm{R}\left(\pi_{\mathbf{X}}\right)_{*} \mathcal{F}$. Le lemme suivant montre quel parti on peut tirer du fait que ce morphisme est un isomorphisme.

Lemme 12.3. Supposons que $\mathbf{X}$ est quasi-affine, purement de dimension d, que $\Lambda$ est un corps et que $D(\mathcal{F})$ a ses faisceaux de cohomologie nuls en dehors du degré $-2 d$.

Si can cast $_{\mathcal{F}}$ un isomorphisme, alors, $\operatorname{can}_{\mathcal{H}^{-2 d} D(\mathcal{F})}$ est un isomorphisme et $H_{c}^{i}(\mathbf{X}, \mathcal{F})=0$ pour $i \neq d$.

Démonstration. Soit $j: \mathbf{X} \rightarrow \mathbf{Y}$ une immersion ouverte avec $\mathbf{Y}$ affine. On a un diagramme commutatif, où les flèches sont les morphismes canoniques

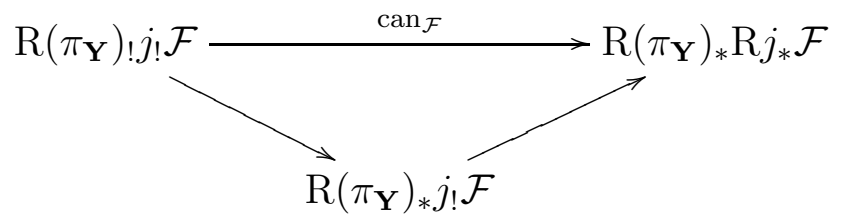

Puisque $\mathbf{Y}$ est affine, on a $H^{i}\left(\mathbf{Y}, j_{!} \mathcal{F}\right)=0$ pour $i \notin[0 \ldots d]$, donc $H_{c}^{i}(\mathbf{X}, \mathcal{F})=0$ pour $i \notin[0 \ldots d]$.

On a (dualité de Verdier=adjonction entre $\mathrm{R}\left(\pi_{\mathbf{X}}\right)$ ! et $\pi_{\mathbf{X}}^{!}$)

$$
H_{c}^{i}(\mathbf{X}, \mathcal{G}) \simeq H^{2 d-i}(\mathbf{X}, \mathcal{F})^{*}
$$

où $\mathcal{G}=\mathcal{H}^{-2 d} D(\mathcal{F})$. Par conséquent, la proposition résultera de ce que can cast $_{\text {un }}$ isomorphisme.

Nous allons déduire ceci d'un résultat plus général. Soit $f: \mathbf{X}_{1} \rightarrow \mathbf{X}_{2}$ un morphisme de variétés. Soit $C_{i} \in D_{\Lambda}^{b}\left(\mathbf{X}_{i}\right)$.

Nous allons maintenant rappeler une construction classique, qui s'effectue en étudiant séparément le cas où $f$ est propre et le cas où $f$ est une immersion ouverte (cf [KaScha, exercice III.9 (iii)] pour le cas de la topologie classique).

On a un diagramme commutatif

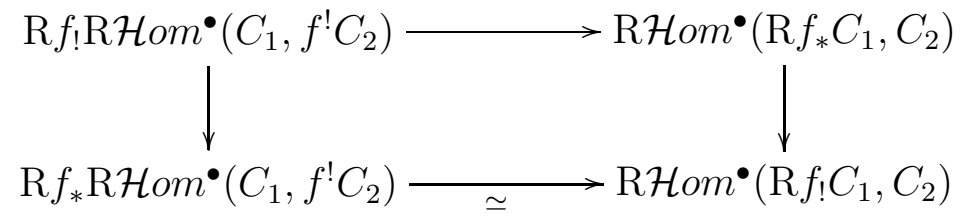

où les flèches verticales proviennent des morphismes canoniques $\mathrm{R} f_{!} \rightarrow \mathrm{R} f_{*}$, la seconde flèche horizontale provient de l'adjonction entre $\mathrm{R} f$ ! et $f$ ! 
On déduit du diagramme précédent un diagramme commutatif

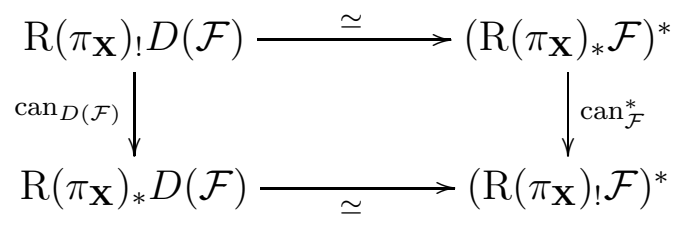

Par conséquent, $\operatorname{can}_{D(\mathcal{F})}$ est un isomorphisme, donc $\operatorname{can}_{\mathcal{G}}$ aussi.

Remarque 12.4. Dans $[\mathrm{Ha}]$, Haastert démontre ce résultat en supposant que $\operatorname{can}_{D(\mathcal{F})}$ est un isomorphisme.

Remarque 12.5. Si, dans le lemme 12.3, on suppose que $\mathbf{X}$ est affine et non pas seulement quasi-affine, alors la conclusion est bien connue.

Passons maintenant à l'application qui nous est utile.

Soit $G$ un groupe fini agissant sur $\mathbf{X}$.

Lemme 12.6. Supposons la variété $\mathbf{X}$ quasi-affine, lisse, purement de dimension d, et supposons que $\Lambda$ est un corps. Supposons de plus que les stabilisateurs de points de $\mathbf{X}$ dans $G$ sont d'ordre inversible dans $\Lambda$.

Soit $M$ un $\Lambda G$-module tel que le morphisme canonique $\mathrm{R} \Gamma_{c}(\mathbf{X}) \otimes_{\Lambda G}^{\mathrm{L}} M \rightarrow \mathrm{R} \Gamma(\mathbf{X}) \otimes_{\Lambda G}^{\mathrm{L}} M$ est un isomorphisme. Alors, le complexe $\mathrm{R} \Gamma_{c}(\mathbf{X}) \otimes_{\Lambda G}^{\mathrm{L}} M$ n'a de l'homologie qu'en degré $d$.

Démonstration. Soit $\pi: \mathbf{X} \rightarrow \mathbf{X} / G$ le morphisme quotient et $\mathcal{F}=\pi_{*} \Lambda_{\mathbf{X}} \otimes_{\Lambda G} M_{\mathbf{X} / G}$. D'après le lemme 3.2 , le morphisme $\operatorname{can}_{\mathcal{F}}$ est un isomorphisme.

Un calcul simple utilisant les adjonctions entre $\pi_{*}$ et $\pi^{!}$et entre $\otimes$ et Hom fournit

$$
D(\mathcal{F}) \simeq D\left(\pi_{*} \Lambda_{\mathbf{X}} \otimes_{\Lambda G}^{\mathrm{L}} M_{\mathbf{X} / G}\right) \simeq \pi_{*} D\left(\Lambda_{\mathbf{X}}\right) \otimes_{\Lambda G}^{\mathrm{L}}\left(M^{*}\right)_{\mathbf{X} / G}^{\mathrm{op}}
$$

Puisque $\mathbf{X}$ est lisse, on a $D\left(\Lambda_{\mathbf{X}}\right) \simeq \Lambda_{\mathbf{X}}[2 d]$. L'hypothèse sur les stabilisateurs de points de $\mathbf{X}$ permet d'appliquer le lemme 3.2 et donc de conclure que

$$
D(\mathcal{F}) \simeq \pi_{*} \Lambda_{\mathbf{X}} \otimes_{\Lambda G}\left(M^{*}\right)_{\mathbf{X} / G}^{\text {op }}[2 d]
$$

Puisque $\mathbf{X} / G$ est quasi-affine, le lemme 12.3 fournit le résultat.

12.3. Diviseurs à croisements normaux. Soit $\overline{\mathbf{X}}$ une variété lisse et soit $\mathbf{X}$ un ouvert de $\overline{\mathbf{X}}$. Nous noterons $i: \mathbf{X} \hookrightarrow \overline{\mathbf{X}}$ l'immersion ouverte canonique. Nous supposons que le complémentaire de $\mathbf{X}$ dans $\overline{\mathbf{X}}$ est une réunion finie

$$
\bigcup_{i \in I} \mathbf{D}_{i}
$$

où les $\mathbf{D}_{i}$ sont des diviseurs lisses à croisements normaux.

Par ailleurs, si $J \subset I$, nous noterons $\mathbf{X}_{J}=\left(\bigcap_{i \in J} \mathbf{D}_{i}\right)-\left(\bigcup_{i \in I \backslash J} \mathbf{D}_{i}\right)$. Selon les conventions usuelles, $\mathbf{X}_{\emptyset}=\mathbf{X}$ et $\mathbf{X}_{I}=\bigcap_{i \in I} \mathbf{D}_{i}$. Notons $i_{J}: \mathbf{X}_{J} \hookrightarrow \overline{\mathbf{X}}$ l'immersion localement fermée canonique.

Le résultat suivant est classique (cf par exemple [SGA4 $\frac{1}{2},(1.3 .3 .2)$ p. 255]) : 
Lemme 12.7. Si $J \subset I$ et si $n \in \mathbf{N}$, alors

$$
\begin{gathered}
i_{J}^{*} \mathrm{R}^{n} i_{*} \Lambda_{\mathbf{X}} \simeq \Lambda_{\mathbf{X}_{J}}^{\oplus\left(\begin{array}{c}
|J| \\
n
\end{array}\right)} . \\
\text { RÉFÉRENCES }
\end{gathered}
$$

[Bo] C. Bonnafé, Mackey formula in type A, Proc. London Math. Soc. 80 (2000), 545-574.

[Br] M. Broué, Isométries de caractères et équivalences de Morita ou dérivées, Publ. Math. I.H.E.S. 71 (1990), 45-63.

[BrMi] M. Broué et J. Michel, Blocs et séries de Lusztig dans un groupe réductif fini, J. Reine Angew. Math. 395 (1989), 56-67.

[DeLu] P. Deligne et G. Lusztig, Representations of reductive groups over finite fields, Ann. of Math. 103 (1976), 103-161.

[DiMi] F. Digne et J. Michel, Representations of finite groups of Lie type, London Math. Soc. Student Texts 21, Cambridge University Press, 1991.

[DiMiRo] F. Digne, J. Michel et R. Rouquier, Cohomologie de certaines variétés de Deligne-Lusztig attachées à des éléments réguliers, en préparation.

[Ha] B. Hanstert, Die quasiaffinität der Deligne-Lusztig Varietäten, J. of Alg. 102 (1986), 186-193.

[Hi] G. Hiss, On the decomposition numbers of $G_{2}(q)$, J. of Alg. 120 (1989), 339-360.

[KaScha] M. Kashiwara et P. Schapira, "Sheaves on manifolds", Springer Verlag, 1990.

[Lu1] G. Lusztig, On the finiteness of the number of unipotent classes, Invent. Math. 34 (1976), 201-213.

[Lu2] G. LuszTig, Green functions and character sheaves, Ann. of Math. 131 (1990), 355-408.

[Mi] J.S. Milne, Étale cohomology, Princeton University Press, 1980.

[SGA4 $\left.\frac{1}{2}\right]$ P. Deligne et al., "SGA4 $\frac{1}{2}$ Cohomologie étale", Springer Lecture Notes in Math. 569, 1977.

[Ti] J. Tits, Normalisateurs de tores. I. Groupes de Coxeter étendus, J. Algebra 4 (1966), 96-116.

Cédric Bonnafé : Université de Franche-Comté, Département de Mathématiques (CNRS UMR 6623), 16 Route De Gray, 25000 BesanÇON, FRANCE.

E-mail : bonnafe@math.univ-fcomte.fr

RaphaËl Rouquier : UFR de Mathématiques et Institut de Mathématiques de Jussieu (CNRS UMR 7586), Université Paris 7, 2 Place Jussieu, 75251 Paris Cedex 05, FRANCE.

E-mail : rouquier@math.jussieu.fr 